\title{
Copper(I)-Catalyzed Chlorine Atom Transfer Radical Cyclization Reactions of Unsaturated $\alpha$-Chloro $\beta$-Keto Esters
}

Dan Yang, ${ }^{*}$ Yi-Long Yan, Bao-Fu Zheng, Qiang Gao and Nian-Yong Zhu Department of Chemistry, The University of Hong Kong, Pokfulam Road, Hong Kong yangdan@hku.hk

\section{Supporting Information}

Table of Content

\begin{tabular}{|l|r|}
\hline General Procedure & S2 \\
\hline Preparation of Substrates & S3-17 \\
\hline NOESY Spectrum of Compound 4f & S18 \\
\hline X-Ray Analysis of Compound 3b & S19-25 \\
\hline X-Ray Analysis of Compound 3g & S26-32 \\
\hline NMR Spectra & S33-74 \\
\hline
\end{tabular}




\section{General procedure:}

All reactions were performed in oven-dried flasks. THF was distilled from sodium metalbenzophenone ketyl before use. Flash column chromatography was performed on E. Merck silica gel 60 (230-400 mesh ASTM) using ethyl acetate/n-hexane as eluting solvents. Nuclear magnetic resonance spectra were recorded in deuteriochloroform $\left(\mathrm{CDCl}_{3}\right)$ with tetramethylsilane (TMS) as the internal standard at ambient temperature on a $300 \mathrm{MHz}$ or $400 \mathrm{MHz}$ Bruker Avance Fourier Transform Spectrometer. Infrared absorption spectra were recorded as a solution in $\mathrm{CH}_{2} \mathrm{Cl}_{2}$ with a Bio-Rad FTS 165 Fourier Tranform Spectrophotometer. Mass spectra were recorded with a Finnigan MAT 95 mass spectrometer for both low resolution and high-resolution mass spectra. Melting points were determined by Axiolab ZEISS microscope apparatus and were uncorrected. Optical rotations were recorded on a Perkin Elmer 343 Polarimeter. 


\section{Preparation of Substrates 1a-f}



$5(\mathbf{a}: \mathrm{R}=\mathrm{Me}, \mathbf{c}: \mathrm{R}=\mathrm{H}, \mathbf{b}, \mathbf{d}-\mathbf{f}: \mathrm{R}=\mathrm{Cl})$

6

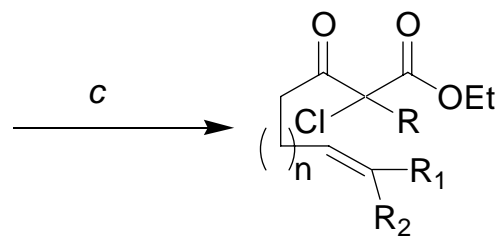

$1 \mathbf{a}-\mathbf{f}$

Reagents and conditions: (a) $\mathrm{NaH}, n-\mathrm{BuLi}$, THF, $0^{\circ} \mathrm{C}$; (b) alkyl bromide, $0^{\circ} \mathrm{C}$, overall yield $30-$ $60 \%$ for two steps (a and b); (c) Method A: NaH, NCS, THF, $0^{\circ} \mathrm{C}, 70-90 \%$ yield; Method B: NCS, $\mathrm{Mg}\left(\mathrm{ClO}_{4}\right)_{2}$, EtOAc, rt, yield 70-90\%.<smiles>CC(=O)C(Cl)C(=O)[O-]</smiles><smiles>CCCCC</smiles><smiles>CCOC(=O)C(C(=O)CC)C(=O)CC</smiles>

$1 c$

Typical procedure for alkylation of $\beta$-keto ester dianion (Preparation of 1c): To a suspension of $\mathrm{NaH}(60 \%$ oil dispersion, $1.41 \mathrm{~g}, 59 \mathrm{mmol})$ in THF $(100 \mathrm{~mL})$ was added ethyl 2chloroacetoacetate $(6.1 \mathrm{~mL}, 43 \mathrm{mmol})$ slowly at $0{ }^{\circ} \mathrm{C}$. After $30 \mathrm{~min}, n$-BuLi (1.42 $\mathrm{M}$ in hexane, $50 \mathrm{~mL}, 47 \mathrm{mmol})$ was added slowly at $0{ }^{\circ} \mathrm{C} .4$-Bromo-2-methyl-2-butene (90\%, $5.0 \mathrm{~mL}, 39$ mmol) was added dropwise $0.5 \mathrm{~h}$ later. The reaction was then stirred at room temperature for $7 \mathrm{~h}$. After removal of solvents, the residue was diluted with water and extracted with $\mathrm{Et}_{2} \mathrm{O}$. The combined extracts were washed with water, dried over $\mathrm{MgSO}_{4}$, and then concentrated. The crude product was purified by flash column chromatography to give 1c $(4.56 \mathrm{~g}, 19.6 \mathrm{mmol}, 46 \%)$ as a light yellow oil. Analytical TLC (silica gel 60), 5\% EtOAc in $n$-hexane, $\mathrm{R}_{f}=0.38 ;{ }^{1} \mathrm{H}$ NMR (300 $\left.\mathrm{MHz}, \mathrm{CDCl}_{3}\right) \delta 12.4(\mathrm{~s}, 0.2 \times 1 \mathrm{H}$, enol), $5.17-5.04(\mathrm{~m}, 1 \mathrm{H}), 4.78(\mathrm{~s}$, keto, $0.8 \times 1 \mathrm{H}), 4.29(\mathrm{q}, J=$ $7.2 \mathrm{~Hz}, 2 \mathrm{H}), 2.82-2.64(\mathrm{~m}, 0.8 \times 2 \mathrm{H}), 2.56-2.39(\mathrm{~m}, 0.2 \times 2 \mathrm{H}), 2.34-2.27(\mathrm{~m}, 2 \mathrm{H}), 1.68(\mathrm{~s}, 3 \mathrm{H})$, 
$1.62(\mathrm{~s}, 3 \mathrm{H}), 1.32(\mathrm{t}, J=7.1 \mathrm{~Hz}, 3 \mathrm{H}) ;{ }^{13} \mathrm{C} \mathrm{NMR}\left(68 \mathrm{MHz}, \mathrm{CDCl}_{3}\right.$; DEPT) major $\delta 198.7(\mathrm{C})$, $165.1(\mathrm{C}), 133.5(\mathrm{C}), 121.8(\mathrm{CH}), 63.1\left(\mathrm{CH}_{2}\right), 61.1(\mathrm{CH}), 39.1\left(\mathrm{CH}_{2}\right), 25.7\left(\mathrm{CH}_{3}\right), 22.3\left(\mathrm{CH}_{2}\right)$, $17.7\left(\mathrm{CH}_{3}\right), 14.0\left(\mathrm{CH}_{3}\right)$; IR $\left(\mathrm{CH}_{2} \mathrm{Cl}_{2}\right) 2972,2933,1760,1730,1604 \mathrm{~cm}^{-1}$; LRMS for $\mathrm{C}_{11} \mathrm{H}_{17} \mathrm{ClO}_{3}$ (EI, $20 \mathrm{eV}$ ) m/z $197\left(\mathrm{M}^{+}-\mathrm{Cl}, 50\right), 151$ (44), 49 (100); HRMS (EI) for $\mathrm{C}_{11} \mathrm{H}_{17} \mathrm{O}_{3}\left(\mathrm{M}^{+}-\mathrm{Cl}\right)$ : calcd 197.1178, found 197.1169.

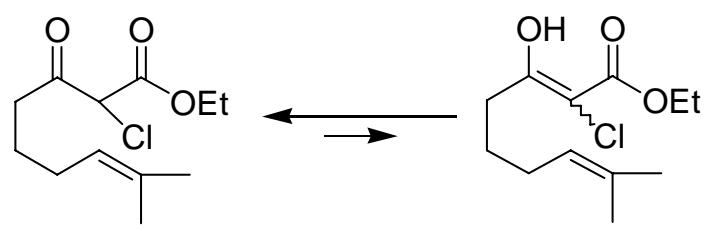

\section{Compound 6d}

Compound 6d: Prepared similarly to 1c. Yield 21\%, a light yellow oil; analytical TLC (silica gel 60), $60 \%$ EtOAc in $n$-hexane, $\mathrm{R}_{f}=0.38 ;{ }^{1} \mathrm{H}$ NMR $\left(300 \mathrm{MHz}, \mathrm{CDCl}_{3}\right) \delta 12.42(\mathrm{~s}, 0.2 \times 1 \mathrm{H}$, enol), $5.14-5.05(\mathrm{~m}, 1 \mathrm{H}), 4.78(\mathrm{~s}$, keto, $0.8 \times 1 \mathrm{H}), 4.29(\mathrm{q}, J=7.1 \mathrm{~Hz}, 2 \mathrm{H}), 2.78-2.70(\mathrm{~m}, 2 \mathrm{H}), 2.09-$ $1.97(\mathrm{~m}, 2 \mathrm{H}), 1.62-1.72(\mathrm{~m}, 2 \mathrm{H}), 1.69(\mathrm{~s}, 3 \mathrm{H}), 1.59(\mathrm{~s}, 3 \mathrm{H}), 1.31(\mathrm{t}, J=7.1 \mathrm{~Hz}, 3 \mathrm{H}) ;{ }^{13} \mathrm{C} \mathrm{NMR}$ (75 MHz, $\mathrm{CDCl}_{3}$; DEPT) $\delta 199.1(\mathrm{C}), 166.1(\mathrm{C}), 132.9(\mathrm{C}), 123.3(\mathrm{CH}), 63.1(\mathrm{CH}), 61.0\left(\mathrm{CH}_{2}\right)$, $38.4\left(\mathrm{CH}_{2}\right), 27.1\left(\mathrm{CH}_{2}\right), 25.7\left(\mathrm{CH}_{3}\right), 23.7\left(\mathrm{CH}_{2}\right), 17.7\left(\mathrm{CH}_{3}\right), 14.0\left(\mathrm{CH}_{3}\right)$; IR $\left(\mathrm{CH}_{2} \mathrm{Cl}_{2}\right)$ 2977, 2939, 1765, $1729 \mathrm{~cm}^{-1}$; LRMS for $\mathrm{C}_{12} \mathrm{H}_{19} \mathrm{ClO}_{3}(\mathrm{EI}, 20 \mathrm{eV}) \mathrm{m} / \mathrm{z} 248\left(\mathrm{M}^{+}, 3\right), 246\left(\mathrm{M}^{+}, 9\right), 211$ $\left(\mathrm{M}^{+}-\mathrm{Cl}, 100\right), 210\left(\mathrm{M}^{+}-\mathrm{HCl}, 24\right), 165$ (45); $\mathrm{HRMS}(\mathrm{EI})$ for $\mathrm{C}_{12} \mathrm{H}_{19} \mathrm{ClO}_{3}\left(\mathrm{M}^{+}\right)$: calcd 246.1023, found 246.1017 . 


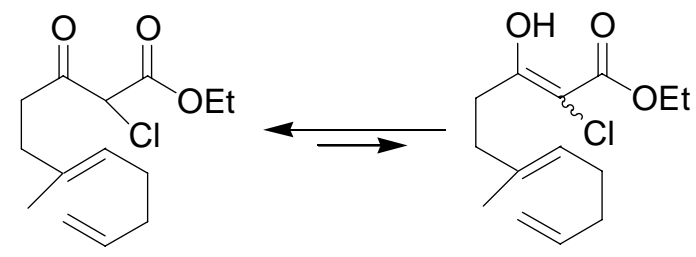

\section{Compound $6 \mathbf{f}$}

Compound 6f: Prepared similarly to 1c. Yield 61\%, a colorless yellow oil; analytical TLC (silica gel 60), 10\% EtOAc in $n$-hexane, $\mathrm{R}_{f}=0.44 ;{ }^{1} \mathrm{H}$ NMR $\left(300 \mathrm{MHz}, \mathrm{CDCl}_{3}\right) \delta 12.39(\mathrm{~s}, 0.3 \times 1 \mathrm{H}$, enol), $5.85-5.76(\mathrm{~m}, 1 \mathrm{H}), 5.19-5.15(\mathrm{~m}, 1 \mathrm{H}), 5.03-4.91(\mathrm{~m}, 2 \mathrm{H}), 4.79(\mathrm{~s}, 0.7 \times 1 \mathrm{H}, \mathrm{keto}), 4.29$ $(\mathrm{q}, J=7.1 \mathrm{~Hz}, 2 \mathrm{H}), 2.87-2.59(\mathrm{~m}, 2 \mathrm{H}), 2.30(\mathrm{t}, J=7.2 \mathrm{~Hz}, 2 \mathrm{H}), 2.09-2.07(\mathrm{~m}, 4 \mathrm{H}), 1.68-$ $1.61(\mathrm{~m}, 3 \mathrm{H}), 1.38-1.29(2 \mathrm{t}, J=7.1 \mathrm{~Hz}, 3 \mathrm{H}) ;{ }^{13} \mathrm{C} \mathrm{NMR}\left(75 \mathrm{MHz}, \mathrm{CDCl}_{3}\right.$; DEPT) $\delta$ major 198.9 (C), $165.4(\mathrm{C}), 138.7(\mathrm{CH}), 133.5(\mathrm{C}), 125.4(\mathrm{CH}), 114.9\left(\mathrm{CH}_{2}\right), 63.4\left(\mathrm{CH}_{2}\right), 61.3(\mathrm{CH}), 38.1$ $\left(\mathrm{CH}_{2}\right), 34.1\left(\mathrm{CH}_{2}\right), 33.5\left(\mathrm{CH}_{2}\right), 27.7\left(\mathrm{CH}_{2}\right), 16.4\left(\mathrm{CH}_{3}\right), 14.3\left(\mathrm{CH}_{3}\right)$; IR $\left(\mathrm{CH}_{2} \mathrm{Cl}_{2}\right)$ 2980, 2932, 1752, 1730, $1640 \mathrm{~cm}^{-1}$; LRMS for $\mathrm{C}_{14} \mathrm{H}_{21} \mathrm{ClO}_{3}(\mathrm{EI}, 20 \mathrm{eV}) \mathrm{m} / \mathrm{z} 272\left(\mathrm{M}^{+}, 3\right), 237\left(\mathrm{M}^{+}-\mathrm{Cl}, 42\right)$, 205 (61), 191 (52), 177 (58), 109 (100); HRMS (EI) for $\mathrm{C}_{14} \mathrm{H}_{21} \mathrm{ClO}_{3}\left(\mathrm{M}^{+}\right)$: calcd 272.1179, found 272.1176.



\section{Compound $6 \mathbf{g}$}

Compound 6g: Prepared similarly to 1c. Yield 40\%, a light yellow oil; analytical TLC (silica gel 60), 10\% EtOAc in $n$-hexane, $\mathrm{R}_{f}=0.47 ;{ }^{1} \mathrm{H}$ NMR $\left(300 \mathrm{MHz}, \mathrm{CDCl}_{3}\right) \delta 12.39(\mathrm{~s}, 0.4 \times 1 \mathrm{H}$, enol), $5.87-5.73(\mathrm{~m}, 1 \mathrm{H}), 5.21-5.14(\mathrm{~m}, 1 \mathrm{H}), 4.96-4.92(\mathrm{~m}, 2 \mathrm{H}), 4.79(\mathrm{~s}, 0.6 \times 1 \mathrm{H}$, keto $), 4.29(\mathrm{q}, J$ 
$=7.2 \mathrm{~Hz}, 2 \mathrm{H}), 2.84-2.77(\mathrm{~m}, 1 \mathrm{H}), 2.64-2.59(\mathrm{~m}, 1 \mathrm{H}), 2.37-2.27(\mathrm{~m}, 2 \mathrm{H}), 2.07-1.96(\mathrm{~m}$, 4H), $1.73-1.60\left(\mathrm{~m}, 3 \mathrm{H}\right.$, keto and enol $\left.\mathrm{CH}_{3}\right), 1.47-1.29(\mathrm{~m}, 5 \mathrm{H}) ;{ }^{13} \mathrm{C} \mathrm{NMR}\left(75 \mathrm{MHz}, \mathrm{CDCl}_{3}\right.$; DEPT) keto and enol $\delta 198.9$ (C), 175.7 (C, enol), 169.9 (C, enol), 165.4 (C), $139.2(\mathrm{CH}), 133.7$ (C), $126.0(\mathrm{CH}), 114.8\left(\mathrm{CH}_{2}\right), 114.7\left(\mathrm{CH}_{2}\right), 139.1(\mathrm{CH}), 133.3(\mathrm{C}), 63.4\left(\mathrm{CH}_{2}\right), 62.3\left(\mathrm{CH}_{2}\right), 61.3$ (CH), $38.1\left(\mathrm{CH}_{2}\right), 35.8\left(\mathrm{CH}_{2}\right), 33.6\left(3 \times \mathrm{CH}_{2}\right), 32.3\left(\mathrm{CH}_{2}\right), 29.2\left(\mathrm{CH}_{2}\right), 27.7\left(\mathrm{CH}_{2}\right), 16.3\left(\mathrm{CH}_{3}\right)$, $16.2\left(\mathrm{CH}_{3}\right), 14.5\left(\mathrm{CH}_{3}\right), 14.3\left(\mathrm{CH}_{3}\right)$; IR $\left(\mathrm{CH}_{2} \mathrm{Cl}_{2}\right) 3078,2984,2934,1732,1642 \mathrm{~cm}^{-1}$; LRMS for $\mathrm{C}_{15} \mathrm{H}_{23} \mathrm{ClO}_{3}\left(\mathrm{EI}, 20 \mathrm{eV}\right.$ ) m/z $286\left(\mathrm{M}^{+}, 9\right), 268$ (9), 251 (70), 205 (87), 164 (76), 122 (52); HRMS (EI) for $\mathrm{C}_{15} \mathrm{H}_{23} \mathrm{ClO}_{3}\left(\mathrm{M}^{+}\right)$: calcd 286.1336, found 286.1331.

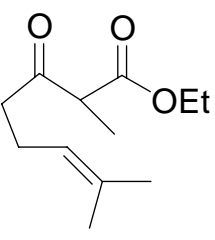

$6 a$

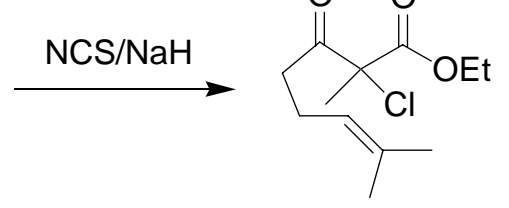

$1 \mathrm{a}$

Typical procedure for $\alpha$-chlorination of olefinic $\beta$-keto esters by NCS/NaH (method A): To a suspension of $\mathrm{NaH}(60 \%$ oil dispersion, $790 \mathrm{mg}, 19.8 \mathrm{mmol})$ in $\mathrm{THF}(50 \mathrm{~mL})$ was added slowly the THF $(10 \mathrm{~mL})$ solution of compound $6 \mathbf{a}(3.50 \mathrm{~g}, 16.5 \mathrm{mmol})$ at $0^{\circ} \mathrm{C}$. After $30 \mathrm{~min}, \mathrm{~N}$ chlorosuccinimide $(2.48 \mathrm{~g}, 18.2 \mathrm{mmol})$ was added in one portion at $0^{\circ} \mathrm{C}$. The mixture was stirred for $3.5 \mathrm{~h}$. After removal of THF, the residue was extracted with $\mathrm{Et}_{2} \mathrm{O}$. The combined extracts were washed with water, dried over $\mathrm{MgSO}_{4}$ and then concentrated. The crude product was purified by flash column chromatography to give 1a $(3.52 \mathrm{~g}, 14.3 \mathrm{mmol}, 86 \%)$ as a colorless oil. Analytical TLC (silica gel 60), 10\% EtOAc in $n$-hexane, $\mathrm{R}_{f}=0.48 ;{ }^{1} \mathrm{H} \mathrm{NMR}\left(300 \mathrm{MHz}, \mathrm{CDCl}_{3}\right)$ $\delta 5.07(\mathrm{t}, J=7.2 \mathrm{~Hz}, 1 \mathrm{H}), 4.27(\mathrm{q}, J=7.1 \mathrm{~Hz}, 2 \mathrm{H}), 2.86-2.75(\mathrm{~m}, 1 \mathrm{H}), 2.71-2.61(\mathrm{~m}, 1 \mathrm{H})$, $2.30(\mathrm{q}, J=7.2 \mathrm{~Hz}, 2 \mathrm{H}), 1.82(\mathrm{~s}, 3 \mathrm{H}), 1.68(\mathrm{~s}, 3 \mathrm{H}), 1.62(\mathrm{~s}, 3 \mathrm{H}), 1.30(\mathrm{t}, J=7.1 \mathrm{~Hz}, 3 \mathrm{H}) ;{ }^{13} \mathrm{C}$ 
NMR (75 MHz, $\mathrm{CDCl}_{3}$; DEPT) $\delta 201.0$ (C), 168.1 (C), 133.2 (C), 122.2 (C), 70.9 (C), 63.0 $\left(\mathrm{CH}_{2}\right), 37.7\left(\mathrm{CH}_{2}\right), 25.7\left(\mathrm{CH}_{3}\right), 24.4\left(\mathrm{CH}_{3}\right), 22.8\left(\mathrm{CH}_{2}\right), 17.7\left(\mathrm{CH}_{3}\right), 13.9\left(\mathrm{CH}_{3}\right)$; IR $\left(\mathrm{CH}_{2} \mathrm{Cl}_{2}\right)$ 2973, 1750, $1730 \mathrm{~cm}^{-1}$; LRMS for $\mathrm{C}_{12} \mathrm{H}_{19} \mathrm{ClO}_{3}(\mathrm{EI}, 20 \mathrm{eV}) \mathrm{m} / \mathrm{z} 245\left(\mathrm{M}^{+}-\mathrm{H}, 14\right), 211\left(\mathrm{M}^{+}-\mathrm{Cl}\right.$, 5), 136 (20), 69 (100); HRMS (EI) for $\mathrm{C}_{12} \mathrm{H}_{19} \mathrm{O}_{3}\left(\mathrm{M}^{+}-\mathrm{Cl}\right)$ : calcd 211.1334, found 211.1343.<smiles>CCOC(=O)C(C(=O)CCC1=CCCC=C1)C(=O)OCC</smiles>

$6 f$

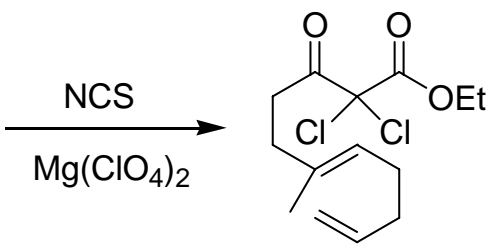

$1 f$

Typical procedure for the Lewis acid-catalyzed $\alpha$-chlorination of olefinic $\beta$-keto ester with NCS (method B): To a stirred solution of substrate $6 \mathbf{f}(630 \mathrm{mg}, 2.3 \mathrm{mmol})$ in EtOAc $(30 \mathrm{~mL})$ was added Lewis acid $\mathrm{Mg}\left(\mathrm{ClO}_{4}\right)_{2}(268 \mathrm{mg}, 1.2 \mathrm{mmol})$ at room temperature. Solid NCS (370 mg, $0.28 \mathrm{mmol}$ ) was added to the above mixture $5 \mathrm{~min}$ later. The reaction was completed $1 \mathrm{~h}$ later. The reaction mixture was diluted with $\mathrm{Et}_{2} \mathrm{O}$ and washed with water then dried over $\mathrm{MgSO}_{4}$. After concentration, the crude product was purified by flash column chromatography using EtOAc/ $n$-hexane as eluents to give 1f $(605 \mathrm{mg}, 85 \%)$ as a colorless oil; Analytical TLC (silica gel 60), 10\% EtOAc in $n$-hexane, $\mathrm{R}_{f}=0.38 ;{ }^{1} \mathrm{H}$ NMR $\left(300 \mathrm{MHz}, \mathrm{CDCl}_{3}\right) \delta 5.84-5.76(\mathrm{~m}, 1 \mathrm{H})$, $5.20-5.18(\mathrm{~m}, 1 \mathrm{H}), 5.04-4.93(\mathrm{~m}, 2 \mathrm{H}), 4.37(\mathrm{q}, J=7.1 \mathrm{~Hz}, 2 \mathrm{H}), 2.94-2.87(\mathrm{~m}, 2 \mathrm{H}), 2.35(\mathrm{t}, J$ $=7.6 \mathrm{~Hz}, 2 \mathrm{H}), 2.09-2.07(\mathrm{~m}, 4 \mathrm{H}), 1.63(\mathrm{~s}, 3 \mathrm{H}), 1.35(\mathrm{t}, J=7.1 \mathrm{~Hz}, 3 \mathrm{H}) ;{ }^{13} \mathrm{C} \mathrm{NMR}(75 \mathrm{MHz}$, $\mathrm{CDCl}_{3}$; DEPT) $\delta 194.1(\mathrm{C}), 163.8(\mathrm{C}), 138.7(\mathrm{CH}), 133.3(\mathrm{C}), 125.7(\mathrm{CH}), 114.9\left(\mathrm{CH}_{2}\right), 82.2(\mathrm{C})$, $64.9\left(\mathrm{CH}_{2}\right), 35.1\left(\mathrm{CH}_{2}\right), 34.3\left(\mathrm{CH}_{2}\right), 34.0\left(\mathrm{CH}_{2}\right), 27.7\left(\mathrm{CH}_{2}\right), 16.3\left(\mathrm{CH}_{3}\right), 14.1\left(\mathrm{CH}_{3}\right) ; \mathrm{IR}\left(\mathrm{CH}_{2} \mathrm{Cl}_{2}\right)$ 2984, $1755 \mathrm{~cm}^{-1}$; LRMS for $\mathrm{C}_{14} \mathrm{H}_{20} \mathrm{Cl}_{2} \mathrm{O}_{3}(\mathrm{EI}, 20 \mathrm{eV}) \mathrm{m} / \mathrm{z} 306\left(\mathrm{M}^{+}, 1\right), 273\left(\mathrm{M}^{+}-\mathrm{Cl}, 2\right), 271$ $\left(\mathrm{M}^{+}-\mathrm{Cl}, 5\right), 241$ (14), 239 (20), 151 (29), 109 (100); HRMS (EI) for $\mathrm{C}_{14} \mathrm{H}_{20} \mathrm{Cl}_{2} \mathrm{O}_{3}\left(\mathrm{M}^{+}\right)$: calcd 306.0790, found 306.0778. 




Compound 1b

Compound 1b: Prepared from 1c similarly to 1a (method A). Yield 82\%, a light yellow oil; analytical TLC (silica gel 60), 10\% EtOAc in $n$-hexane, $\mathrm{R}_{f}=0.63 ;{ }^{1} \mathrm{H} \mathrm{NMR}\left(300 \mathrm{MHz}, \mathrm{CDCl}_{3}\right) \delta$ $5.09(\mathrm{t}, J=7.2 \mathrm{~Hz}, 1 \mathrm{H}), 4.36(\mathrm{q}, J=7.3 \mathrm{~Hz}, 2 \mathrm{H}), 2.84(\mathrm{t}, J=7.3 \mathrm{~Hz}, 2 \mathrm{H}), 2.36(\mathrm{q}, J=7.2 \mathrm{~Hz}$, 2H), $1.68(\mathrm{~s}, 3 \mathrm{H}), 1.63(\mathrm{~s}, 3 \mathrm{H}), 1.35(\mathrm{t}, J=7.1 \mathrm{~Hz}, 3 \mathrm{H}) ;{ }^{13} \mathrm{C} \mathrm{NMR}\left(75 \mathrm{MHz}, \mathrm{CDCl}_{3}\right.$; DEPT) $\delta$ $194.1(\mathrm{C}), 163.8(\mathrm{C}), 134.0(\mathrm{C}), 121.9(\mathrm{CH}), 82.3(\mathrm{C}), 64.9\left(\mathrm{CH}_{2}\right), 36.4\left(\mathrm{CH}_{2}\right), 26.0\left(\mathrm{CH}_{3}\right), 23.3$ $\left(\mathrm{CH}_{2}\right), 18.0\left(\mathrm{CH}_{3}\right), 14.1\left(\mathrm{CH}_{3}\right)$; IR $\left(\mathrm{CH}_{2} \mathrm{Cl}_{2}\right) 2974,1765,1749 \mathrm{~cm}^{-1}$; LRMS for $\mathrm{C}_{11} \mathrm{H}_{16} \mathrm{Cl}_{2} \mathrm{O}_{3}(\mathrm{EI}$, $20 \mathrm{eV}) \mathrm{m} / \mathrm{z} 265\left(\mathrm{M}^{+}-\mathrm{H}, 13\right), 231\left(\mathrm{M}^{+}-\mathrm{Cl}, 69\right), 230\left(\mathrm{M}^{+}-\mathrm{HCl}, 24\right), 195$ (24), 143 (100); HRMS (EI) for $\mathrm{C}_{11} \mathrm{H}_{16} \mathrm{Cl}_{2} \mathrm{O}_{3}\left(\mathrm{M}^{+}\right)$: calcd 266.0477, found 266.0468 .

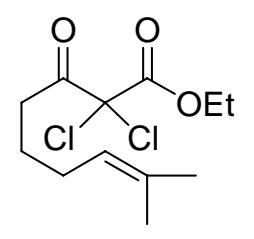

\section{Compound 1d}

Compound 1d: Prepared from 6d similarly to 1a using method A. Yield 76\%, a light yellow oil; analytical TLC (silica gel 60), 5\% EtOAc in $n$-hexane, $\mathrm{R}_{f}=0.56 ;{ }^{1} \mathrm{H}$ NMR $\left(400 \mathrm{MHz}, \mathrm{CDCl}_{3}\right) \delta$ $5.11-5.06(\mathrm{~m}, 1 \mathrm{H}), 4.36(\mathrm{q}, J=7.2 \mathrm{~Hz}, 2 \mathrm{H}), 2.82(\mathrm{t}, J=7.3 \mathrm{~Hz}, 2 \mathrm{H}), 2.03(\mathrm{q}, J=7.2 \mathrm{~Hz}, 2 \mathrm{H})$, $1.76-1.71(\mathrm{~m}, 2 \mathrm{H}), 1.70(\mathrm{~s}, 3 \mathrm{H}), 1.69(\mathrm{~s}, 3 \mathrm{H}), 1.35(\mathrm{t}, J=7.1 \mathrm{~Hz}, 3 \mathrm{H}) ;{ }^{13} \mathrm{C} \mathrm{NMR}(100 \mathrm{MHz}$, $\mathrm{CDCl}_{3}$; DEPT) $\delta 194.6(\mathrm{C}), 163.8(\mathrm{C}), 133.3(\mathrm{C}), 123.4(\mathrm{CH}), 82.2(\mathrm{C}), 64.9\left(\mathrm{CH}_{2}\right), 35.5\left(\mathrm{CH}_{2}\right)$, 
$27.3\left(\mathrm{CH}_{2}\right), 26.0\left(\mathrm{CH}_{3}\right), 24.7\left(\mathrm{CH}_{2}\right), 18.0\left(\mathrm{CH}_{3}\right), 14.1\left(\mathrm{CH}_{3}\right)$; IR $\left(\mathrm{CH}_{2} \mathrm{Cl}_{2}\right) 2970,1765,1748 \mathrm{~cm}^{-1}$; LRMS for $\mathrm{C}_{12} \mathrm{H}_{19} \mathrm{Cl}_{2} \mathrm{O}_{3}$ (EI, $\left.20 \mathrm{eV}\right) \mathrm{m} / \mathrm{z} 280\left(\mathrm{M}^{+}, 1\right), 245\left(\mathrm{M}^{+}-\mathrm{Cl}, 69\right), 244\left(\mathrm{M}^{+}-\mathrm{HCl}, 24\right), 209$ (42), 163 (34), 125 (100); HRMS (EI) for $\mathrm{C}_{12} \mathrm{H}_{18} \mathrm{Cl}_{2} \mathrm{O}_{3}\left(\mathrm{M}^{+}\right)$: calcd 280.0633, found 280.0631.

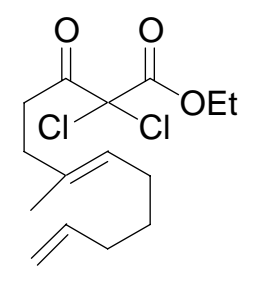

Compound 1g

Compound 1g: Prepared from $\mathbf{6 g}$ similarly to $\mathbf{1 f}$ using method B. Yield 61\%, light yellow oil; analytical TLC (silica gel 60), 10\% EtOAc in $n$-hexane, $\mathrm{R}_{f}=0.46 ;{ }^{1} \mathrm{H} \mathrm{NMR}\left(300 \mathrm{MHz}, \mathrm{CDCl}_{3}\right) \delta$ $5.85-5.75(\mathrm{~m}, 1 \mathrm{H}), 5.19(\mathrm{t}, J=7.2 \mathrm{~Hz}, 1 \mathrm{H}), 5.03-4.93(\mathrm{~m}, 2 \mathrm{H}), 4.37$ (q, $J=7.2 \mathrm{~Hz}, 2 \mathrm{H}), 2.92$ (t, $J=8.0 \mathrm{~Hz}, 2 \mathrm{H}), 2.33(\mathrm{t}, J=7.7 \mathrm{~Hz}, 2 \mathrm{H}), 2.08-1.96(\mathrm{~m}, 4 \mathrm{H}), 1.62(\mathrm{~s}, 3 \mathrm{H}), 1.35(\mathrm{t}, J=7.1 \mathrm{~Hz}$, 3H), $1.43(\mathrm{p}, J=7.3 \mathrm{~Hz}, 2 \mathrm{H}) ;{ }^{13} \mathrm{C}$ NMR (75 MHz, $\mathrm{CDCl}_{3}$; DEPT) $\delta 194.1$ (C), 163.8 (C), 139.1 $(\mathrm{CH}), 133.1(\mathrm{C}), 126.3(\mathrm{CH}), 114.8(\mathrm{CH}), 82.2(\mathrm{C}), 64.9\left(\mathrm{CH}_{2}\right), 36.1\left(\mathrm{CH}_{2}\right), 34.3\left(\mathrm{CH}_{2}\right), 33.6$ $\left(\mathrm{CH}_{2}\right), 29.2\left(\mathrm{CH}_{2}\right), 27.7\left(\mathrm{CH}_{2}\right), 16.3\left(\mathrm{CH}_{3}\right), 14.1\left(\mathrm{CH}_{3}\right)$; IR $\left(\mathrm{CH}_{2} \mathrm{Cl}_{2}\right) 2980,2932,1754,1642$ $\mathrm{cm}^{-1}$; LRMS for $\mathrm{C}_{15} \mathrm{H}_{22} \mathrm{Cl}_{2} \mathrm{O}_{3}(\mathrm{EI}, 20 \mathrm{eV}) \mathrm{m} / \mathrm{z} 287\left(\mathrm{M}^{+}-\mathrm{Cl}, 7\right), 285\left(\mathrm{M}^{+}-\mathrm{Cl}, 27\right), 249$ (6), 239 (45), 203 (30), 165 (100); HRMS (EI) for $\mathrm{C}_{15} \mathrm{H}_{22} \mathrm{ClO}_{3}\left(\mathrm{M}^{+}-\mathrm{Cl}\right)$ : calcd 285.1257, found 285.1256. 


\section{Preparation of Substrate 1e}<smiles>COC(=O)CC(C)=O</smiles>

$5 e$

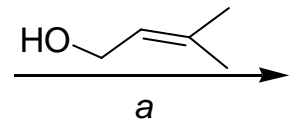<smiles>CC(=O)CC(=O)OCC=C(C)C(C)(C)C</smiles>

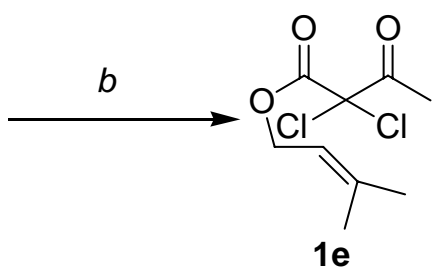

Reagents and conditions: (a) 3-Methyl-2-buten-1-ol, DMAP, toluene, reflux, 60\% yield; (b) NCS (2.5 equiv), $\mathrm{Mg}\left(\mathrm{ClO}_{4}\right)_{2}$ (0.5 equiv), EtOAc, rt, $83 \%$ yield.

Preparation of 6e: Methyl acetoacetate (2.35 g, 20 mmol), 3-methyl-2-buten-1-ol (2.07 g, 24 mmol) and DMAP (1.22 g, $10 \mathrm{mmol})$ were mixed in toluene $(30 \mathrm{~mL})$. The mixture was heated to reflux for $20 \mathrm{~h}$. After removal of solvent, the residue was extracted with $\mathrm{Et}_{2} \mathrm{O}$. The extract was washed with water and then dried over $\mathrm{MgSO}_{4}$. After concentration, the crude product was purified by flash column chromatography to give 6 e $(2.07 \mathrm{~g}, 60 \%)$ as light yellow oil. Analytical TLC (silica gel 60), 10\% EtOAc in $n$-hexane, $\mathrm{R}_{f}=0.32 ;{ }^{1} \mathrm{H}$ NMR $\left(300 \mathrm{MHz}, \mathrm{CDCl}_{3}\right) \delta 12.09(\mathrm{~s}$, 0.1×1H, enol), 5.35 (t, $J=7.3 \mathrm{~Hz}, 1 \mathrm{H}), 4.98(\mathrm{~s}, 0.1 \times 1 \mathrm{H}$, enol), $4.64(\mathrm{~d}, J=7.3 \mathrm{~Hz}, 2 \mathrm{H}), 3.45(\mathrm{~s}$,

$0.9 \times 2 \mathrm{H}$, keto), $2.27(\mathrm{~s}, 3 \mathrm{H}), 1.77(\mathrm{~s}, 3 \mathrm{H}), 1.72(\mathrm{~s}, 3 \mathrm{H}) ;{ }^{13} \mathrm{C} \mathrm{NMR}\left(75 \mathrm{MHz}, \mathrm{CDCl}_{3} ; \mathrm{DEPT}\right) \delta$ major $200.9(\mathrm{C}), 167.4(\mathrm{C}), 140.1(\mathrm{C}), 118.3(\mathrm{CH}), 62.5\left(\mathrm{CH}_{2}\right), 50.4\left(\mathrm{CH}_{2}\right), 30.3\left(\mathrm{CH}_{3}\right), 21.4$ $\left(\mathrm{CH}_{3}\right), 18.3\left(\mathrm{CH}_{3}\right)$; IR $\left(\mathrm{CH}_{2} \mathrm{Cl}_{2}\right) 2976,1741,1716,1649 \mathrm{~cm}^{-1}$; LRMS for $\mathrm{C}_{9} \mathrm{H}_{14} \mathrm{O}_{3}(\mathrm{EI}, 20 \mathrm{eV})$ m/z $170\left(\mathrm{M}^{+}, 4\right), 169\left(\mathrm{M}^{+}-\mathrm{H}, 100\right), 153,147$; HRMS (EI) for $\mathrm{C}_{9} \mathrm{H}_{14} \mathrm{O}_{3}\left(\mathrm{M}^{+}\right)$: calcd 170.0943, found 170.0944 . 


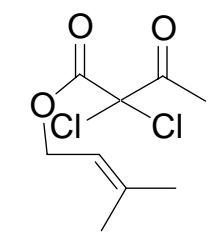

Compound 1e

Compound 1e: Prepared from 6e similarly to 1f using method B with 2.5 equiv of NCS. Yield $83 \%$, light yellow oil; analytical TLC (silica gel 60), 5\% EtOAc in $n$-hexane, $\mathrm{R}_{f}=0.35 ;{ }^{1} \mathrm{H}$ NMR $\left(300 \mathrm{MHz}, \mathrm{CDCl}_{3}\right) \delta 5.37(\mathrm{t}, J=7.4 \mathrm{~Hz}, 1 \mathrm{H}), 4.79(\mathrm{~d}, J=7.3 \mathrm{~Hz}, 2 \mathrm{H}), 2.47(\mathrm{~s}, 3 \mathrm{H}), 1.78(\mathrm{~s}, 3 \mathrm{H})$, $1.74(\mathrm{~s}, 3 \mathrm{H}) ;{ }^{13} \mathrm{C}$ NMR $\left(75 \mathrm{MHz}, \mathrm{CDCl}_{3}\right.$; DEPT) $\delta 191.4(\mathrm{C}), 163.6(\mathrm{C}), 142.2(\mathrm{C}), 116.9(\mathrm{CH})$, $80.8(\mathrm{C}), 65.5\left(\mathrm{CH}_{2}\right), 26.0\left(\mathrm{CH}_{3}\right), 23.8\left(\mathrm{CH}_{3}\right), 18.4\left(\mathrm{CH}_{3}\right)$; IR $\left(\mathrm{CH}_{2} \mathrm{Cl}_{2}\right) 2969,1756,1725 \mathrm{~cm}^{-1}$; LRMS for $\mathrm{C}_{9} \mathrm{H}_{12} \mathrm{Cl}_{2} \mathrm{O}_{3}$ (EI, $\left.20 \mathrm{eV}\right)$ m/z $205\left(\mathrm{M}^{+}-\mathrm{Cl}, 32\right), 203\left(\mathrm{M}^{+}-\mathrm{Cl}, 45\right), 153$ (94), 136 (100), 106 (59); HRMS (EI) for $\mathrm{C}_{9} \mathrm{H}_{12} \mathrm{ClO}_{3}\left(\mathrm{M}^{+}-\mathrm{Cl}\right)$ : calcd 203.0475, found 203.0463.

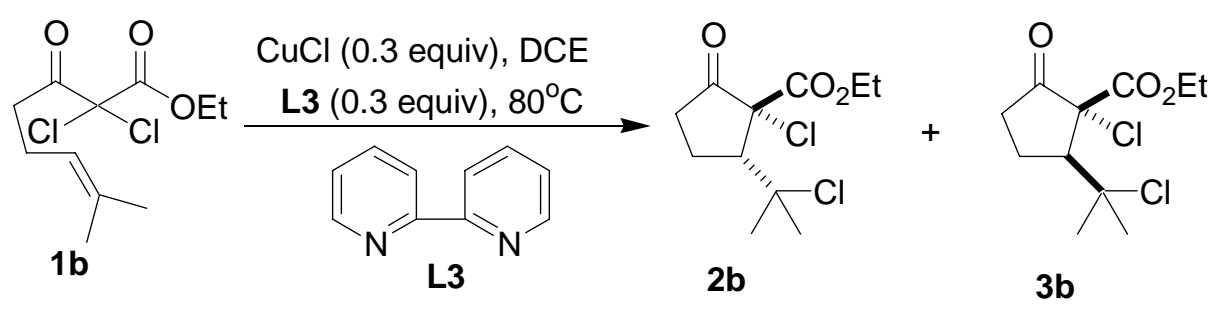

Typical procedure for $\mathrm{CuCl}$ catalyzed chlorine transfer radical cyclization reactions (entry 11, Table 2): A solution of $\mathbf{1 b}(80 \mathrm{mg}, 0.3 \mathrm{mmol})$ in 1,2-dichloroethane (DCE, $10 \mathrm{~mL})$ was deoxygenated with argon at room temperature. Bipyridine ligand L3 (16.6 mg, $0.1 \mathrm{mmol}$ ) was added followed by copper (I) chloride $(10 \mathrm{mg}, 0.1 \mathrm{mmol})$. The reaction heated to $80^{\circ} \mathrm{C}$ (oil-bath) and stirred in a sealed system. The reaction finished $24 \mathrm{~h}$ later. The mixture was diluted with $\mathrm{Et}_{2} \mathrm{O}$ and washed with water. The organic layer was dried over $\mathrm{MgSO}_{4}$ and concentrated. The crude product was purified by column chromatography to give $\mathbf{2 b}(21.8 \mathrm{mg}, 27 \%)$ and $\mathbf{3 b}(32.0$ $\mathrm{mg}, 40 \%)$. 
Compound 2b: A colorless oil; analytical TLC (silica gel 60), 10\% EtOAc in $n$-hexane, $\mathrm{R}_{f}=0.32$; ${ }^{1} \mathrm{H}$ NMR $\left(400 \mathrm{MHz}, \mathrm{CDCl}_{3}\right) \delta 4.32(\mathrm{q}, J=7.3 \mathrm{~Hz}, 2 \mathrm{H}), 3.39(\mathrm{dd}, J=5.9,12.63 \mathrm{~Hz}, 1 \mathrm{H}), 2.79-$ $2.72(\mathrm{~m}, 1 \mathrm{H}), 2.52-2.42(\mathrm{~m}, 1 \mathrm{H}), 2.39-2.32(\mathrm{~m}, 1 \mathrm{H}), 2.26-2.17(\mathrm{~m}, 1 \mathrm{H}), 1.74(\mathrm{~s}, 3 \mathrm{H}), 1.71(\mathrm{~s}$, 3H), $1.33(\mathrm{t}, J=7.1 \mathrm{~Hz}, 3 \mathrm{H}) ;{ }^{13} \mathrm{C}$ NMR (100 MHz, $\mathrm{CDCl}_{3}$; DEPT) $\delta 204.5$ (C), $167.8(\mathrm{C}), 73.1$ (C), $70.2(\mathrm{C}), 63.8\left(\mathrm{CH}_{2}\right), 57.9(\mathrm{CH}), 35.8\left(\mathrm{CH}_{2}\right), 32.0\left(\mathrm{CH}_{3}\right), 31.5\left(\mathrm{CH}_{3}\right), 22.3\left(\mathrm{CH}_{2}\right), 14.3$ $\left(\mathrm{CH}_{3}\right)$; IR $\left(\mathrm{CH}_{2} \mathrm{Cl}_{2}\right) 2984,1770,1752 \mathrm{~cm}^{-1}$; LRMS for $\mathrm{C}_{11} \mathrm{H}_{16} \mathrm{Cl}_{2} \mathrm{O}_{3}(\mathrm{EI}, 20 \mathrm{eV}) \mathrm{m} / \mathrm{z} 268\left(\mathrm{M}^{+}, 4\right)$, $266\left(\mathrm{M}^{+}, 6\right), 230\left(\mathrm{M}^{+}-\mathrm{HCl}, 3\right), 195$ (54), 149 (100); HRMS (EI) for $\mathrm{C}_{11} \mathrm{H}_{16} \mathrm{Cl}_{2} \mathrm{O}_{3}\left(\mathrm{M}^{+}\right)$: calcd 266.0477, found 266.0475 .

Compound 3b: A colorless oil (crystallized slowly, m. p. $78-79^{\circ} \mathrm{C}$ ); analytical TLC (silica gel 60), $10 \%$ EtOAc in $n$-hexane, $\mathrm{R}_{f}=0.25 ;{ }^{1} \mathrm{H} \mathrm{NMR}\left(400 \mathrm{MHz}, \mathrm{CDCl}_{3}\right) \delta 4.29-4.14(\mathrm{~m}, 2 \mathrm{H}), 2.84$ $-2.76(\mathrm{~m}, 1 \mathrm{H}), 2.73-2.68(\mathrm{~m}, 1 \mathrm{H}), 2.54-2.39(\mathrm{~m}, 2 \mathrm{H}), 2.36-2.30(\mathrm{~m}, 1 \mathrm{H}), 1.86(\mathrm{~s}, 3 \mathrm{H})$, $1.70(\mathrm{~s}, 3 \mathrm{H}), 1.29(\mathrm{t}, J=7.2 \mathrm{~Hz}, 3 \mathrm{H}) ;{ }^{13} \mathrm{C} \mathrm{NMR}\left(100 \mathrm{MHz}, \mathrm{CDCl}_{3}\right.$; DEPT) $\delta 206.7(\mathrm{C}), 165.9$ (C), $73.4(\mathrm{C}), 70.5(\mathrm{C}), 63.6\left(\mathrm{CH}_{2}\right), 62.8(\mathrm{CH}), 35.7\left(\mathrm{CH}_{2}\right), 34.1\left(\mathrm{CH}_{3}\right), 31.3\left(\mathrm{CH}_{3}\right), 22.6\left(\mathrm{CH}_{2}\right)$, $14.0\left(\mathrm{CH}_{3}\right)$; IR $\left(\mathrm{CH}_{2} \mathrm{Cl}_{2}\right) 2983,1771,1752 \mathrm{~cm}^{-1}$; LRMS for $\mathrm{C}_{11} \mathrm{H}_{16} \mathrm{Cl}_{2} \mathrm{O}_{3}(\mathrm{EI}, 20 \mathrm{eV}) \mathrm{m} / \mathrm{z} 268$ $\left(\mathrm{M}^{+}, 9\right), 266\left(\mathrm{M}^{+}, 15\right), 230\left(\mathrm{M}^{+}-\mathrm{HCl}, 7\right), 195$ (76), 149 (100); HRMS (EI) for $\mathrm{C}_{11} \mathrm{H}_{16} \mathrm{Cl}_{2} \mathrm{O}_{3}\left(\mathrm{M}^{+}\right)$: calcd 266.0477, found 266.0473. 


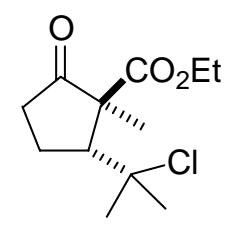

Compound 2a

Compound 2a: A colorless oil; analytical TLC ( silica gel 60), 10\% EtOAc in $n$-hexane, $\mathrm{R}_{f}=0.22$; ${ }^{1} \mathrm{H}$ NMR $\left(300 \mathrm{MHz}, \mathrm{CDCl}_{3}\right) \delta 4.21(\mathrm{q}, J=7.1 \mathrm{~Hz}, 2 \mathrm{H}), 3.12(\mathrm{dd}, J=6.0,12.7 \mathrm{~Hz}, 1 \mathrm{H}), 2.61-$ $2.40(\mathrm{~m}, 2 \mathrm{H}), 2.37-2.23(\mathrm{~m}, 1 \mathrm{H}), 2.13-1.98(\mathrm{~m}, 1 \mathrm{H}), 1.66(\mathrm{~s}, 3 \mathrm{H}), 1.60(\mathrm{~s}, 3 \mathrm{H}), 1.38(\mathrm{~s}, 3 \mathrm{H})$, $1.25(\mathrm{t}, J=7.1 \mathrm{~Hz}, 3 \mathrm{H}) ;{ }^{13} \mathrm{C} \mathrm{NMR}\left(75 \mathrm{MHz}, \mathrm{CDCl}_{3}\right.$; DEPT) $\delta 214.5$ (C), $172.6(\mathrm{C}), 70.6$ (C), $61.7\left(\mathrm{CH}_{2}\right), 60.6(\mathrm{C}), 55.5(\mathrm{CH}), 36.9\left(\mathrm{CH}_{2}\right), 33.2\left(\mathrm{CH}_{3}\right), 31.5\left(\mathrm{CH}_{3}\right), 22.3\left(\mathrm{CH}_{2}\right), 14.6\left(\mathrm{CH}_{3}\right)$, $14.1\left(\mathrm{CH}_{3}\right)$; IR $\left(\mathrm{CH}_{2} \mathrm{Cl}_{2}\right) 2984,1753,1728 \mathrm{~cm}^{-1}$; LRMS for $\mathrm{C}_{12} \mathrm{H}_{19} \mathrm{ClO}_{3}(\mathrm{EI}, 20 \mathrm{eV}) \mathrm{m} / \mathrm{z} 248\left(\mathrm{M}^{+}\right.$, 4), $246\left(\mathrm{M}^{+}, 4\right), 210\left(\mathrm{M}^{+}-\mathrm{HCl}, 17\right), 182$ (14), 155 (100); HRMS (EI) for $\mathrm{C}_{12} \mathrm{H}_{19} \mathrm{ClO}_{3}\left(\mathrm{M}^{+}\right)$: calcd 246.1023, found 246.1019.



Compound 2d

Compound 2d: A light yellow oil; analytical TLC (silica gel 60), 10\% EtOAc in $n$-hexane, $\mathrm{R}_{f}=$ 0.33; ${ }^{1} \mathrm{H}$ NMR $\left(300 \mathrm{MHz}, \mathrm{CDCl}_{3}\right) \delta 4.39-4.20(\mathrm{~m}, 2 \mathrm{H}), 3.17(\mathrm{dd}, J=3.3,12.0 \mathrm{~Hz}, 1 \mathrm{H}), 2.94$ $(\mathrm{dt}, J=6.2,14.4 \mathrm{~Hz}, 1 \mathrm{H}), 2.40-2.48(\mathrm{~m}, 1 \mathrm{H}), 2.29-2.22(\mathrm{~m}, 1 \mathrm{H}), 2.19-2.10(\mathrm{~m}, 1 \mathrm{H}), 2.09-$ $1.98(\mathrm{~m}, 1 \mathrm{H}), 1.76-1.61(\mathrm{~m}, 1 \mathrm{H}), 1.73(\mathrm{~s}, 3 \mathrm{H}), 1.68(\mathrm{~s}, 3 \mathrm{H}), 1.35(\mathrm{t}, J=7.1 \mathrm{~Hz}, 3 \mathrm{H}) ;{ }^{13} \mathrm{C} \mathrm{NMR}$ (75 MHz, $\mathrm{CDCl}_{3}$; DEPT) $\delta 199.6(\mathrm{C}), 168.2(\mathrm{C}), 76.5(\mathrm{C}), 72.7(\mathrm{C}), 63.4\left(\mathrm{CH}_{2}\right), 54.7(\mathrm{CH}), 36,4$ $\left(\mathrm{CH}_{2}\right), 32.0\left(\mathrm{CH}_{3}\right), 31.6\left(\mathrm{CH}_{3}\right), 24.2\left(\mathrm{CH}_{2}\right), 23.6\left(\mathrm{CH}_{2}\right), 14.2\left(\mathrm{CH}_{3}\right) ; \mathrm{IR}\left(\mathrm{CH}_{2} \mathrm{Cl}_{2}\right) 2984,1756$, 
$1723 \mathrm{~cm}^{-1}$; LRMS for $\mathrm{C}_{12} \mathrm{H}_{18} \mathrm{Cl}_{2} \mathrm{O}_{3}(\mathrm{EI}, 20 \mathrm{eV}) \mathrm{m} / \mathrm{z} 282\left(\mathrm{M}^{+}, 23\right), 280\left(\mathrm{M}^{+}, 36\right), 245\left(\mathrm{M}^{+}-\mathrm{Cl}, 7\right)$, $244\left(\mathrm{M}^{+}-\mathrm{HCl}, 22\right), 209$ (97), 163 (100); HRMS (EI) for $\mathrm{C}_{12} \mathrm{H}_{18} \mathrm{Cl}_{2} \mathrm{O}_{3}\left(\mathrm{M}^{+}\right)$: calcd 280.0633, found 280.0651 .



Compound 3d

Compound 3d: A light yellow oil; analytical TLC (silica gel 60), 10\% EtOAc in $n$-hexane, $\mathrm{R}_{f}=$ 0.28; ${ }^{1} \mathrm{H}$ NMR $\left(300 \mathrm{MHz}, \mathrm{CDCl}_{3}\right) \delta 4.15-4.34(\mathrm{~m}, 2 \mathrm{H}), 2.82(\mathrm{~m}, 2 \mathrm{H}), 2.58(\mathrm{dd}, J=3.5,11.7$ $\mathrm{Hz}, 1 \mathrm{H}), 2.35-2.24(\mathrm{~m}, 2 \mathrm{H}), 2.22-2.06(\mathrm{~m}, 1 \mathrm{H}), 1.88-1.78(\mathrm{~m}, 1 \mathrm{H}), 1.82(\mathrm{~s}, 3 \mathrm{H}), 1.80(\mathrm{~s}$, 3H), $1.32(\mathrm{t}, J=7.2 \mathrm{~Hz}, 3 \mathrm{H}) ;{ }^{13} \mathrm{C}$ NMR $\left(75 \mathrm{MHz}, \mathrm{CDCl}_{3}\right.$; DEPT $\delta 200.3(\mathrm{C}), 165.9(\mathrm{C}), 80.8(\mathrm{C})$, $73.2(\mathrm{C}), 63.7\left(\mathrm{CH}_{2}\right), 60.9(\mathrm{CH}), 37.6\left(\mathrm{CH}_{2}\right), 35.3\left(\mathrm{CH}_{3}\right), 31.6\left(\mathrm{CH}_{3}\right), 25.1\left(\mathrm{CH}_{2}\right), 21.7\left(\mathrm{CH}_{2}\right)$, $14.0\left(\mathrm{CH}_{3}\right)$; IR $\left(\mathrm{CH}_{2} \mathrm{Cl}_{2}\right) 2985,1755,1730 \mathrm{~cm}^{-1}$; LRMS for $\mathrm{C}_{12} \mathrm{H}_{19} \mathrm{Cl}_{2} \mathrm{O}_{3}(\mathrm{EI}, 20 \mathrm{eV}) \mathrm{m} / \mathrm{z} 282$ $\left(\mathrm{M}^{+}, 17\right), 280\left(\mathrm{M}^{+}, 26\right), 244\left(\mathrm{M}^{+}-\mathrm{HCl}, 15\right), 209$ (80), 181 (79), 163 (100); HRMS (EI) for $\mathrm{C}_{12} \mathrm{H}_{18} \mathrm{Cl}_{2} \mathrm{O}_{3}\left(\mathrm{M}^{+}\right)$: calcd 280.0633, found 280.0650.

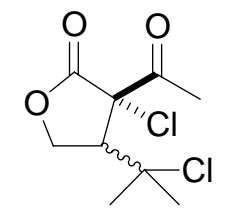

\section{Compound 2e/3e (isomer 1)}

Compound 2e/3e (isomer 1): A light yellow oil; analytical TLC (silica gel 60), 15\% EtOAc in $n$ hexane, $\mathrm{R}_{f}=0.28 ;{ }^{1} \mathrm{H}$ NMR $\left(300 \mathrm{MHz}, \mathrm{CDCl}_{3}\right) \delta 4.62-4.51(\mathrm{~m}, 2 \mathrm{H}), 3.93(\mathrm{dd}, J=7.5,10.1 \mathrm{~Hz}$, 
1H), $2.64(\mathrm{~s}, 3 \mathrm{H}), 1.70(\mathrm{~s}, 6 \mathrm{H}) ;{ }^{13} \mathrm{C} \mathrm{NMR}\left(75 \mathrm{MHz}, \mathrm{CDCl}_{3}\right.$; DEPT) $\delta 200.4(\mathrm{C}), 169.6(\mathrm{C}), 70.6$ (C), $68.1\left(\mathrm{CH}_{2}\right), 66.9(\mathrm{C}), 53.6(\mathrm{CH}), 33.0\left(\mathrm{CH}_{3}\right), 31.6\left(\mathrm{CH}_{3}\right), 27.9\left(\mathrm{CH}_{3}\right)$; IR $\left(\mathrm{CH}_{2} \mathrm{Cl}_{2}\right)$ 2996, 1786, $1723 \mathrm{~cm}^{-1}$; LRMS for $\mathrm{C}_{9} \mathrm{H}_{12} \mathrm{Cl}_{2} \mathrm{O}_{3}$ (EI, $\left.20 \mathrm{eV}\right) \mathrm{m} / \mathrm{z} 239\left(\mathrm{M}^{+}, 1\right), 196$ (20), 121 (29), 119 (100); HRMS (EI) for $\mathrm{C}_{9} \mathrm{H}_{13} \mathrm{Cl}_{2} \mathrm{O}_{3}\left(\mathrm{M}^{+}+\mathrm{H}\right)$ : calcd 239.0242, found 239.0261.

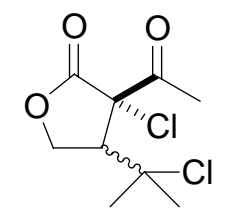

\section{Compound 2e/3e (isomer 2)}

Compound 2e/3e (isomer 2): A light yellow oil; analytical TLC (silica gel 60), 15\% EtOAc in $n$ hexane, $\mathrm{R}_{f}=0.15 ;{ }^{1} \mathrm{H}$ NMR $\left(300 \mathrm{MHz}, \mathrm{CDCl}_{3}\right) \delta 4.62-4.50(\mathrm{~m}, 2 \mathrm{H}), 3.24(\mathrm{dd}, J=7.8,10.6 \mathrm{~Hz}$, 1H), $2.59(\mathrm{~s}, 3 \mathrm{H}), 1.85(\mathrm{~s}, 3 \mathrm{H}), 1.64(\mathrm{~s}, 3 \mathrm{H}) ;{ }^{13} \mathrm{C} \mathrm{NMR}\left(75 \mathrm{MHz}, \mathrm{CDCl}_{3}\right.$; DEPT) $\delta 200.7(\mathrm{C})$, $170.7(\mathrm{C}), 73.3(\mathrm{C}), 67.7\left(\mathrm{CH}_{2}\right), 66.3(\mathrm{C}), 63.2(\mathrm{CH}), 33.2\left(\mathrm{CH}_{3}\right), 32.0\left(\mathrm{CH}_{3}\right), 30.5\left(\mathrm{CH}_{3}\right)$; IR $\left(\mathrm{CH}_{2} \mathrm{Cl}_{2}\right) 2988,1797,1713 \mathrm{~cm}^{-1}$; LRMS for $\mathrm{C}_{9} \mathrm{H}_{12} \mathrm{Cl}_{2} \mathrm{O}_{3}(\mathrm{EI}, 20 \mathrm{eV}) \mathrm{m} / \mathrm{z} 241\left(\mathrm{M}^{+}+\mathrm{H}, 1\right), 239$ $\left(\mathrm{M}^{+}+\mathrm{H}, 1\right), 203\left(\mathrm{M}^{+}-\mathrm{Cl}, 1\right), 196$ (17), 121 (26), 119 (100); HRMS (EI) for $\mathrm{C}_{9} \mathrm{H}_{12} \mathrm{ClO}_{3}\left(\mathrm{M}^{+}\right)$: calcd 239.0242, found 239.0264.

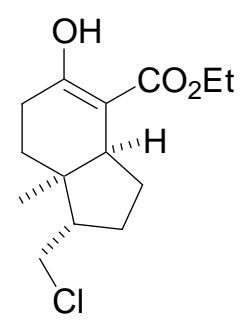

\section{Compound $\mathbf{4 f}$}

Compound 4f: A light yellow oil; analytical TLC (silica gel 60), 15\% EtOAc in $n$-hexane, $\mathrm{R}_{f}=$ 0.56; ${ }^{1} \mathrm{H}$ NMR $\left(300 \mathrm{MHz}, \mathrm{CDCl}_{3}\right) \delta 12.30(\mathrm{~s}, \mathrm{OH}), 4.31-4.11(\mathrm{~m}, 2 \mathrm{H}), 3.67(\mathrm{dd}, J=5.5,10.6$ 
$\mathrm{Hz}, 1 \mathrm{H}), 3.42(\mathrm{dd}, J=7.9,10.6 \mathrm{~Hz}, 1 \mathrm{H}), 2.46-2.37(\mathrm{~m}, 1 \mathrm{H}), 2.34-2.15(\mathrm{~m}, 3 \mathrm{H}), 2.10-1.94$ $(\mathrm{m}, 2 \mathrm{H}), 1.75-1.66(\mathrm{~m}, 1 \mathrm{H}), 1.57-1.39(\mathrm{~m}, 2 \mathrm{H}), 1.31(\mathrm{t}, J=7.1 \mathrm{~Hz}, 3 \mathrm{H}), 1.23-1.32(\mathrm{~m}, 1 \mathrm{H})$ $0.99(\mathrm{~s}, 3 \mathrm{H}) ;{ }^{13} \mathrm{C}$ NMR $\left(75 \mathrm{MHz}, \mathrm{CDCl}_{3}\right.$; DEPT) $\delta 173.3(\mathrm{C}), 171.3(\mathrm{C}), 101.0(\mathrm{C}), 60.6\left(\mathrm{CH}_{2}\right)$, $50.3(\mathrm{CH}), 47.7\left(\mathrm{CH}_{2}\right), 45.1(\mathrm{CH}), 32.5\left(\mathrm{CH}_{2}\right), 32.2\left(\mathrm{CH}_{2}\right), 29.5\left(\mathrm{CH}_{2}\right), 26.5\left(\mathrm{CH}_{2}\right), 20.6\left(\mathrm{CH}_{3}\right)$, 14.6 $\left(\mathrm{CH}_{3}\right)$; IR $\left(\mathrm{CH}_{2} \mathrm{Cl}_{2}\right) 3017,2965,1739,1649 \mathrm{~cm}^{-1}$; LRMS for $\mathrm{C}_{14} \mathrm{H}_{21} \mathrm{ClO}_{3}(\mathrm{EI}, 20 \mathrm{eV}) \mathrm{m} / \mathrm{z}$ $274\left(\mathrm{M}^{+}, 19\right), 272\left(\mathrm{M}^{+}, 65\right), 237\left(\mathrm{M}^{+}-\mathrm{Cl}, 88\right), 225$ (30), 191 (100); HRMS (EI) for $\mathrm{C}_{14} \mathrm{H}_{21} \mathrm{ClO}_{3}$ $\left(\mathrm{M}^{+}\right)$: calcd 272.1179, found 272.1177.

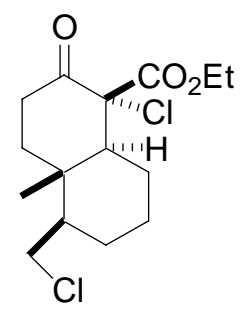

\section{Compound 3g}

Compound 3g: A light yellow oil; analytical TLC (silica gel 60), 15\% EtOAc in $n$-hexane, $\mathrm{R}_{f}=$ 0.31; ${ }^{1} \mathrm{H}$ NMR (300 MHz, $\left.\mathrm{CDCl}_{3}\right) \delta 4.23(\mathrm{q}, J=7.2 \mathrm{~Hz}, 2 \mathrm{H}), 3.73(\mathrm{dd}, J=7.0,10.7 \mathrm{~Hz}, 1 \mathrm{H})$, $3.20(\mathrm{dd}, J=9.6,10.7 \mathrm{~Hz}, 1 \mathrm{H}), 2.96(\mathrm{dt}, J=6.0,14.7 \mathrm{~Hz}, 1 \mathrm{H}), 2.73-2.66(\mathrm{~m}, 1 \mathrm{H}), 2.28-2.23$ (m, 1H), $2.20-2.12(\mathrm{~m}, 1 \mathrm{H}), 2.09-2.02(\mathrm{~m}, 1 \mathrm{H}), 1.99-1.96(\mathrm{~m}, 2 \mathrm{H}), 1.82-1.77(\mathrm{~m}, 1 \mathrm{H})$ $1.63(\mathrm{dd}, J=5.5,14.4 \mathrm{~Hz}, 1 \mathrm{H}), 1.45-1.40(\mathrm{~m}, 1 \mathrm{H}), 1.30(\mathrm{t}, J=7.1 \mathrm{~Hz}, 3 \mathrm{H}), 1.38-1.21(\mathrm{~m}, 2 \mathrm{H})$, 0.95 (s, 3H); ${ }^{13} \mathrm{C}$ NMR (75 MHz, $\mathrm{CDCl}_{3}$; DEPT) $\delta 198.7$ (C), $168.2(\mathrm{C}), 78.2(\mathrm{C}), 63.1\left(\mathrm{CH}_{2}\right)$, $59.3(\mathrm{CH}), 52.8(\mathrm{CH}), 45.9\left(\mathrm{CH}_{2}\right), 39.9(\mathrm{C}), 39.1\left(\mathrm{CH}_{2}\right), 37.1\left(\mathrm{CH}_{2}\right), 26.6\left(\mathrm{CH}_{2}\right), 26.1\left(\mathrm{CH}_{2}\right)$, $24.8\left(\mathrm{CH}_{2}\right), 14.1\left(\mathrm{CH}_{3}\right), 12.7\left(\mathrm{CH}_{3}\right)$; IR $\left(\mathrm{CH}_{2} \mathrm{Cl}_{2}\right)$ 2942, 1744, 1733, $1719 \mathrm{~cm}^{-1}$; LRMS for $\mathrm{C}_{15} \mathrm{H}_{22} \mathrm{Cl}_{2} \mathrm{O}_{3}(\mathrm{EI}, 20 \mathrm{eV}) \mathrm{m} / \mathrm{z} 322\left(\mathrm{M}^{+}, 23\right), 320\left(\mathrm{M}^{+}, 25\right), 285\left(\mathrm{M}^{+}-\mathrm{Cl}, 100\right), 239$ (72), 193 (92), 183 (79); HRMS (EI) for $\mathrm{C}_{15} \mathrm{H}_{22} \mathrm{Cl}_{2} \mathrm{O}_{3}\left(\mathrm{M}^{+}\right)$: calcd 320.0946, found 320.0934. 




Compound $\mathbf{4 g}$

Compound 4g: A colorless oil; analytical TLC (silica gel 60), 20\% EtOAc in $n$-hexane, $\mathrm{R}_{f}=0.30$; ${ }^{1} \mathrm{H}$ NMR $\left(400 \mathrm{MHz}, \mathrm{CDCl}_{3}\right) \delta 4.27-4.19(\mathrm{~m}, 2 \mathrm{H}), 3.79(\mathrm{dd}, J=3.2,10.7 \mathrm{~Hz}, 1 \mathrm{H}), 3.24(\mathrm{dd}, J=$ 9.9, $10.6 \mathrm{~Hz}, 1 \mathrm{H}), 3.21(\mathrm{~d}, J=12.9 \mathrm{~Hz}, 1 \mathrm{H}), 2.47-2.43(\mathrm{~m}, 2 \mathrm{H}), 2.12-2.06(\mathrm{~m}, 2 \mathrm{H}), 2.02-$ $1.94(\mathrm{~m}, 1 \mathrm{H}), 1.86-1.81(\mathrm{~m}, 1 \mathrm{H}), 1.67-1.66(\mathrm{~m}, 1 \mathrm{H}), 1.58-1.43(\mathrm{~m}, 1 \mathrm{H}), 1.43-1.23(\mathrm{~m}, 4 \mathrm{H})$ $1.28(\mathrm{t}, J=7.1 \mathrm{~Hz}, 3 \mathrm{H}), 1.02(\mathrm{~s}, 3 \mathrm{H}) ;{ }^{13} \mathrm{C} \mathrm{NMR}\left(75 \mathrm{MHz}, \mathrm{CDCl}_{3}\right.$; DEPT) $\delta 205.4(\mathrm{C}), 170.0(\mathrm{C})$, $61.3\left(\mathrm{CH}_{2}\right), 60.0(\mathrm{CH}), 51.0(\mathrm{CH}), 48.4(\mathrm{CH}), 46.3\left(\mathrm{CH}_{2}\right), 37.8\left(\mathrm{CH}_{2}\right), 37.7\left(\mathrm{CH}_{2}\right), 36.7(\mathrm{C}), 27.3$ $\left(\mathrm{CH}_{2}\right), 25.8\left(\mathrm{CH}_{2}\right), 25.7\left(\mathrm{CH}_{2}\right), 14.5\left(\mathrm{CH}_{3}\right), 12.2\left(\mathrm{CH}_{3}\right)$; IR $\left(\mathrm{CH}_{2} \mathrm{Cl}_{2}\right) 2938,1741,1712 \mathrm{~cm}^{-1}$; LRMS for $\mathrm{C}_{15} \mathrm{H}_{23} \mathrm{ClO}_{3}(\mathrm{EI}, 20 \mathrm{eV}) \mathrm{m} / \mathrm{z} 288\left(\mathrm{M}^{+}, 4\right), 286\left(\mathrm{M}^{+}, 13\right), 251\left(\mathrm{M}^{+}-\mathrm{Cl}, 40\right), 232$ (23), 223 (28), 144 (35), 143 (100); HRMS (EI) for $\mathrm{C}_{15} \mathrm{H}_{23} \mathrm{ClO}_{3}\left(\mathrm{M}^{+}\right)$: calcd 286.1336, found 286.1338. 
Figure S1. The NOESY spectrum of compound $4 \mathrm{f}$

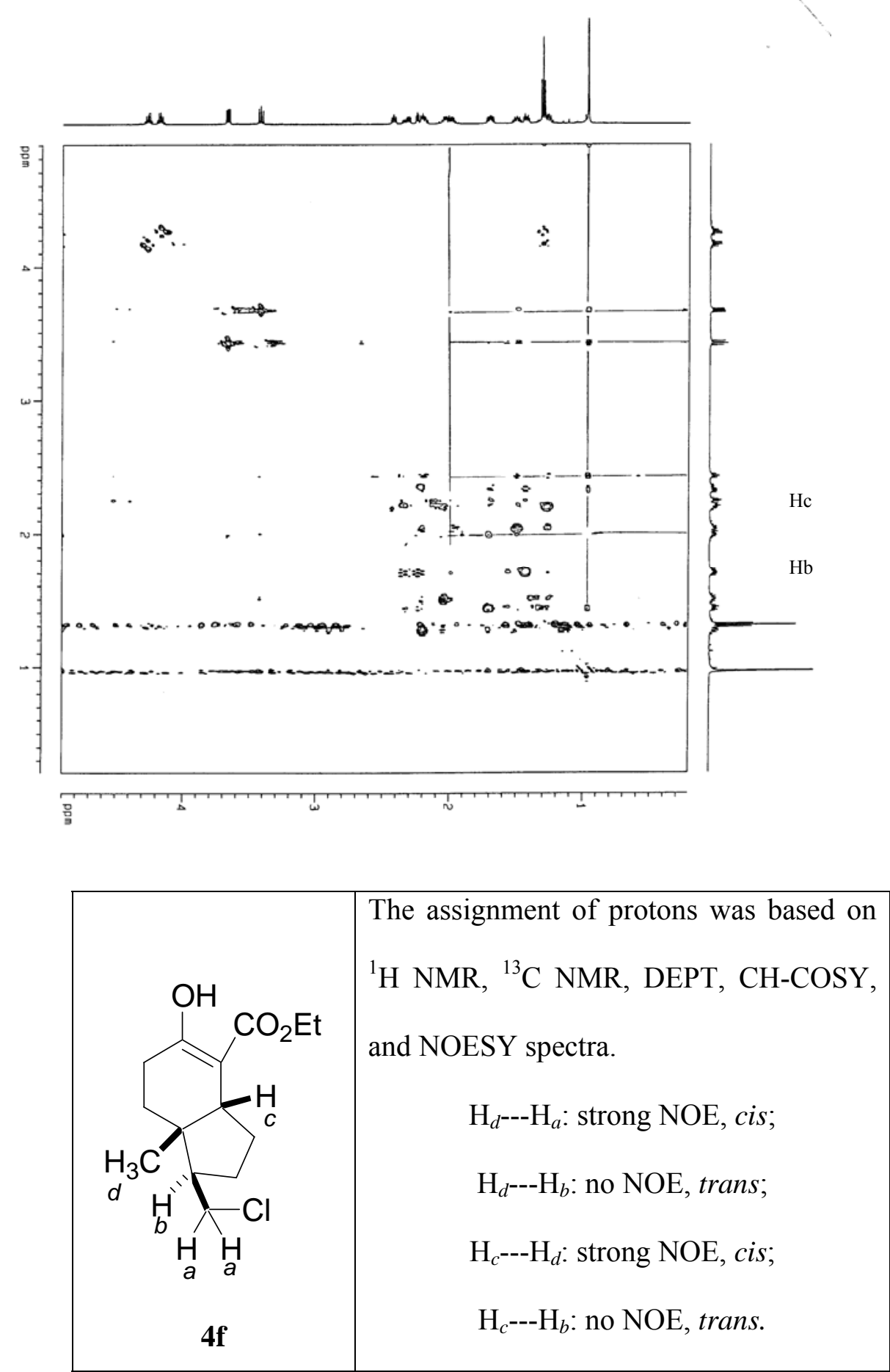


Figure S2. X-ray analysis of compound 3b

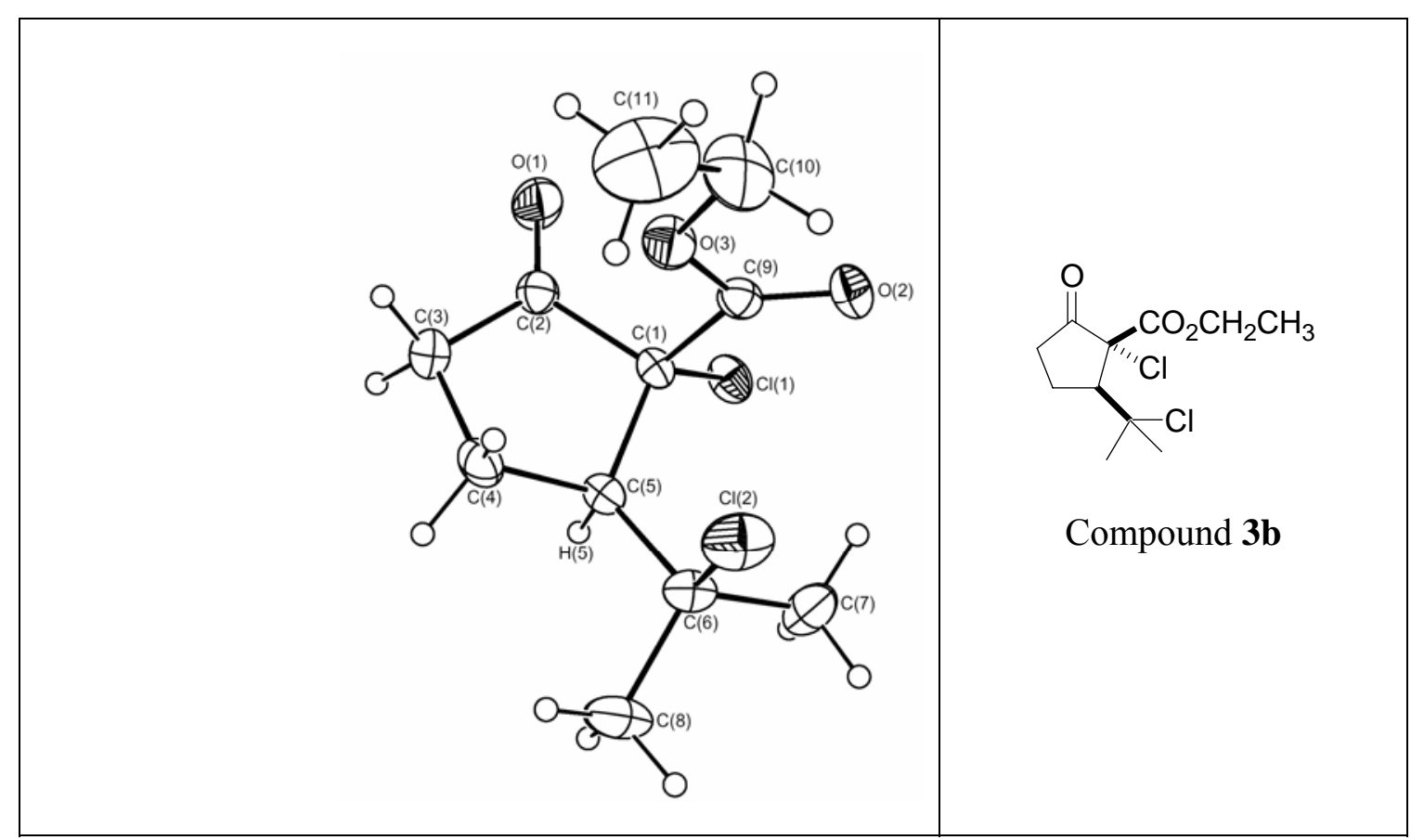


Table 1. Crystal data and structure refinement for mar677a.

Identification code

Empirical formula

Formula weight

Temperature

Wavelength

Crystal system

Space group

Unit cell dimensions

Volume

Z

Density (calculated)

Absorption coefficient

$\mathrm{F}(000)$

Crystal size

Theta range for data collection

Index ranges

Reflections collected

Independent reflections

Completeness to theta $=25.58^{\circ}$

Absorption correction

Max. and min. transmission

Refinement method

Data / restraints / parameters

Goodness-of-fit on $\mathrm{F}^{2}$

Final $\mathrm{R}$ indices [I $>2 \operatorname{sigma}(\mathrm{I})]$

$\mathrm{R}$ indices (all data)

Largest diff. peak and hole mar677a

$\mathrm{C}_{11} \mathrm{H}_{16} \mathrm{Cl}_{2} \mathrm{O}_{3}$

267.14

253(2) K

$0.71073 \AA$

Monoclinic

P $2{ }_{1} / \mathrm{c}$

$\mathrm{a}=13.035(3) \AA$

$\alpha=90^{\circ}$.

$\mathrm{b}=8.1030(16) \AA$

$\beta=97.73(3)^{\circ}$.

$\mathrm{c}=12.377(3) \AA$

$\gamma=90^{\circ}$.

1295.4(4) $\AA^{3}$

4

$1.370 \mathrm{Mg} / \mathrm{m}^{3}$

$0.491 \mathrm{~mm}^{-1}$

560

$0.30 \times 0.20 \times 0.10 \mathrm{~mm}^{3}$

2.97 to $25.58^{\circ}$.

$-15<=\mathrm{h}<=15,-9<=\mathrm{k}<=9,-14<=1<=14$

6828

$2377[\mathrm{R}(\mathrm{int})=0.0326]$

$97.4 \%$

None

0.9525 and 0.8667

Full-matrix least-squares on $\mathrm{F}^{2}$

2377 / 0 / 148

1.137

$\mathrm{R} 1=0.0373, \mathrm{wR} 2=0.1081$

$\mathrm{R} 1=0.0493, \mathrm{wR} 2=0.1123$

0.383 and -0.260 e. $\AA^{-3}$ 
Table 2. Atomic coordinates $\left(\times 10^{4}\right)$ and equivalent isotropic displacement parameters $\left(\AA^{2} \times 10^{3}\right)$ for mar677a. $U(e q)$ is defined as one third of the trace of the orthogonalized $U^{i j}$ tensor.

\begin{tabular}{lrrrr}
\hline & $\mathrm{x}$ & $\mathrm{y}$ & $\mathrm{z}$ & $\mathrm{U}(\mathrm{eq})$ \\
\hline $\mathrm{Cl}(1)$ & & & & \\
$\mathrm{Cl}(2)$ & $4250(1)$ & $164(1)$ & $8048(1)$ & $45(1)$ \\
$\mathrm{O}(1)$ & $1256(1)$ & $-583(1)$ & $9730(1)$ & $66(1)$ \\
$\mathrm{O}(2)$ & $4259(1)$ & $3808(2)$ & $8598(1)$ & $62(1)$ \\
$\mathrm{O}(3)$ & $2046(1)$ & $706(2)$ & $7313(1)$ & $51(1)$ \\
$\mathrm{C}(1)$ & $1895(1)$ & $2755(2)$ & $8495(1)$ & $52(1)$ \\
$\mathrm{C}(2)$ & $3393(1)$ & $1188(2)$ & $8833(1)$ & $33(1)$ \\
$\mathrm{C}(3)$ & $3877(2)$ & $2900(2)$ & $9198(2)$ & $40(1)$ \\
$\mathrm{C}(4)$ & $3798(2)$ & $3154(3)$ & $10376(2)$ & $49(1)$ \\
$\mathrm{C}(5)$ & $3157(2)$ & $1735(2)$ & $10718(2)$ & $45(1)$ \\
$\mathrm{C}(6)$ & $3316(2)$ & $322(2)$ & $9938(1)$ & $35(1)$ \\
$\mathrm{C}(7)$ & $2622(2)$ & $-1193(2)$ & $9987(2)$ & $45(1)$ \\
$\mathrm{C}(8)$ & $2770(2)$ & $-2505(3)$ & $9145(2)$ & $62(1)$ \\
$\mathrm{C}(9)$ & $2794(2)$ & $-1916(3)$ & $11139(2)$ & $69(1)$ \\
$\mathrm{C}(10)$ & $2365(2)$ & $1485(2)$ & $8109(2)$ & $38(1)$ \\
$\mathrm{C}(11)$ & $852(2)$ & $3124(4)$ & $7976(3)$ & $90(1)$ \\
& $325(3)$ & $4031(5)$ & $8751(4)$ & $122(1)$ \\
\hline
\end{tabular}


Table 3. Bond lengths $[\AA]$ and angles $\left[{ }^{\circ}\right]$ for mar677a.

\begin{tabular}{|c|c|c|c|}
\hline $\mathrm{Cl}(1)-\mathrm{C}(1)$ & $1.7817(19)$ & $C(8)-C(6)-C(5)$ & $109.32(18)$ \\
\hline $\mathrm{Cl}(2)-\mathrm{C}(6)$ & $1.833(2)$ & $\mathrm{C}(7)-\mathrm{C}(6)-\mathrm{Cl}(2)$ & $106.22(15)$ \\
\hline $\mathrm{O}(1)-\mathrm{C}(2)$ & $1.200(2)$ & $\mathrm{C}(8)-\mathrm{C}(6)-\mathrm{Cl}(2)$ & $106.43(16)$ \\
\hline $\mathrm{O}(2)-\mathrm{C}(9)$ & $1.196(2)$ & $\mathrm{C}(5)-\mathrm{C}(6)-\mathrm{Cl}(2)$ & $109.99(13)$ \\
\hline $\mathrm{O}(3)-\mathrm{C}(9)$ & $1.320(2)$ & $\mathrm{O}(2)-\mathrm{C}(9)-\mathrm{O}(3)$ & $125.58(18)$ \\
\hline $\mathrm{O}(3)-\mathrm{C}(10)$ & $1.454(3)$ & $\mathrm{O}(2)-\mathrm{C}(9)-\mathrm{C}(1)$ & $125.53(18)$ \\
\hline $\mathrm{C}(1)-\mathrm{C}(9)$ & $1.527(3)$ & $\mathrm{O}(3)-\mathrm{C}(9)-\mathrm{C}(1)$ & $108.89(16)$ \\
\hline$C(1)-C(5)$ & $1.552(2)$ & $\mathrm{C}(11)-\mathrm{C}(10)-\mathrm{O}(3)$ & $108.0(2)$ \\
\hline$C(1)-C(2)$ & $1.565(3)$ & & \\
\hline$C(2)-C(3)$ & $1.489(3)$ & & \\
\hline$C(3)-C(4)$ & $1.515(3)$ & & \\
\hline$C(4)-C(5)$ & $1.530(3)$ & & \\
\hline$C(5)-C(6)$ & $1.531(3)$ & & \\
\hline$C(6)-C(7)$ & $1.519(3)$ & & \\
\hline $\mathrm{C}(6)-\mathrm{C}(8)$ & $1.529(3)$ & & \\
\hline $\mathrm{C}(10)-\mathrm{C}(11)$ & $1.452(4)$ & & \\
\hline $\mathrm{C}(9)-\mathrm{O}(3)-\mathrm{C}(10)$ & $116.92(18)$ & & \\
\hline $\mathrm{C}(9)-\mathrm{C}(1)-\mathrm{C}(5)$ & $115.65(15)$ & & \\
\hline $\mathrm{C}(9)-\mathrm{C}(1)-\mathrm{C}(2)$ & $108.53(15)$ & & \\
\hline$C(5)-C(1)-C(2)$ & $102.63(14)$ & & \\
\hline $\mathrm{C}(9)-\mathrm{C}(1)-\mathrm{Cl}(1)$ & $108.53(12)$ & & \\
\hline $\mathrm{C}(5)-\mathrm{C}(1)-\mathrm{Cl}(1)$ & $112.91(12)$ & & \\
\hline $\mathrm{C}(2)-\mathrm{C}(1)-\mathrm{Cl}(1)$ & $108.16(13)$ & & \\
\hline $\mathrm{O}(1)-\mathrm{C}(2)-\mathrm{C}(3)$ & $127.63(19)$ & & \\
\hline $\mathrm{O}(1)-\mathrm{C}(2)-\mathrm{C}(1)$ & $123.35(18)$ & & \\
\hline$C(3)-C(2)-C(1)$ & $109.01(16)$ & & \\
\hline$C(2)-C(3)-C(4)$ & $106.44(16)$ & & \\
\hline$C(3)-C(4)-C(5)$ & $105.34(15)$ & & \\
\hline$C(4)-C(5)-C(6)$ & $116.34(16)$ & & \\
\hline$C(4)-C(5)-C(1)$ & $104.35(14)$ & & \\
\hline$C(6)-C(5)-C(1)$ & $120.46(16)$ & & \\
\hline$C(7)-C(6)-C(8)$ & $110.82(18)$ & & \\
\hline$C(7)-C(6)-C(5)$ & $113.77(17)$ & & \\
\hline
\end{tabular}


Symmetry transformations used to generate equivalent atoms: 
Table 4. Anisotropic displacement parameters $\left(\AA^{2} \times 10^{3}\right)$ for mar677a. The anisotropic displacement factor exponent takes the form: $-2 \pi^{2}\left[h^{2} a^{* 2} U^{11}+\ldots+2 h k\right.$ a* b* $\left.U^{12}\right]$

\begin{tabular}{lcccccc}
\hline & $\mathrm{U}^{11}$ & $\mathrm{U}^{22}$ & $\mathrm{U}^{33}$ & $\mathrm{U}^{23}$ & $\mathrm{U}^{13}$ & $\mathrm{U}^{12}$ \\
\hline $\mathrm{Cl}(1)$ & $44(1)$ & $57(1)$ & $35(1)$ & $-4(1)$ & $10(1)$ & $8(1)$ \\
$\mathrm{Cl}(2)$ & $42(1)$ & $71(1)$ & $88(1)$ & $19(1)$ & $14(1)$ & $-5(1)$ \\
$\mathrm{O}(1)$ & $75(1)$ & $58(1)$ & $56(1)$ & $2(1)$ & $16(1)$ & $-26(1)$ \\
$\mathrm{O}(2)$ & $49(1)$ & $61(1)$ & $41(1)$ & $-5(1)$ & $-7(1)$ & $-4(1)$ \\
$\mathrm{O}(3)$ & $41(1)$ & $53(1)$ & $59(1)$ & $-2(1)$ & $-5(1)$ & $13(1)$ \\
$\mathrm{C}(1)$ & $34(1)$ & $38(1)$ & $26(1)$ & $-1(1)$ & $3(1)$ & $2(1)$ \\
$\mathrm{C}(2)$ & $38(1)$ & $41(1)$ & $39(1)$ & $0(1)$ & $2(1)$ & $-3(1)$ \\
$\mathrm{C}(3)$ & $61(1)$ & $43(1)$ & $42(1)$ & $-9(1)$ & $3(1)$ & $-2(1)$ \\
$\mathrm{C}(4)$ & $55(1)$ & $52(1)$ & $30(1)$ & $-2(1)$ & $8(1)$ & $7(1)$ \\
$\mathrm{C}(5)$ & $35(1)$ & $39(1)$ & $30(1)$ & $3(1)$ & $4(1)$ & $4(1)$ \\
$\mathrm{C}(6)$ & $46(1)$ & $44(1)$ & $47(1)$ & $11(1)$ & $9(1)$ & $1(1)$ \\
$\mathrm{C}(7)$ & $72(2)$ & $40(1)$ & $74(2)$ & $-2(1)$ & $13(1)$ & $-8(1)$ \\
$\mathrm{C}(8)$ & $79(2)$ & $68(2)$ & $61(2)$ & $32(1)$ & $11(1)$ & $-1(1)$ \\
$\mathrm{C}(9)$ & $37(1)$ & $40(1)$ & $36(1)$ & $5(1)$ & $5(1)$ & $-2(1)$ \\
$\mathrm{C}(10)$ & $56(2)$ & $101(2)$ & $103(2)$ & $-8(2)$ & $-26(2)$ & $37(2)$ \\
$\mathrm{C}(11)$ & $61(2)$ & $123(3)$ & $184(4)$ & $22(3)$ & $24(2)$ & $38(2)$ \\
& & & & & \\
\hline
\end{tabular}


Table 5. Hydrogen coordinates ( $\left.\mathrm{x} 10^{4}\right)$ and isotropic displacement parameters $\left(\AA^{2} \times 10^{3}\right)$ for mar677a.

\begin{tabular}{|c|c|c|c|c|}
\hline & $\mathrm{x}$ & y & $\mathrm{z}$ & $\mathrm{U}(\mathrm{eq})$ \\
\hline $\mathrm{H}(3 \mathrm{~A})$ & 3464 & 4199 & 10485 & 58 \\
\hline $\mathrm{H}(3 \mathrm{~B})$ & 4480 & 3155 & 10799 & 58 \\
\hline $\mathrm{H}(4 \mathrm{~A})$ & 2432 & 2039 & 10656 & 55 \\
\hline $\mathrm{H}(4 \mathrm{~B})$ & 3392 & 1418 & 11467 & 55 \\
\hline $\mathrm{H}(5)$ & 4018 & -80 & 10182 & 42 \\
\hline $\mathrm{H}(7 \mathrm{~A})$ & 2570 & -2067 & 8427 & 93 \\
\hline $\mathrm{H}(7 \mathrm{~B})$ & 2348 & -3447 & 9252 & 93 \\
\hline $\mathrm{H}(7 \mathrm{C})$ & 3484 & -2830 & 9224 & 93 \\
\hline $\mathrm{H}(8 \mathrm{~A})$ & 3516 & -2159 & 11337 & 104 \\
\hline $\mathrm{H}(8 \mathrm{~B})$ & 2398 & -2911 & 11159 & 104 \\
\hline $\mathrm{H}(8 \mathrm{C})$ & 2578 & -1131 & 11643 & 104 \\
\hline $\mathrm{H}(10 \mathrm{~A})$ & 879 & 3781 & 7325 & 108 \\
\hline $\mathrm{H}(10 \mathrm{~B})$ & 483 & 2109 & 7767 & 108 \\
\hline $\mathrm{H}(11 \mathrm{~A})$ & 329 & 3391 & 9404 & 183 \\
\hline $\mathrm{H}(11 \mathrm{~B})$ & -377 & 4243 & 8437 & 183 \\
\hline $\mathrm{H}(11 \mathrm{C})$ & 675 & 5059 & 8923 & 183 \\
\hline
\end{tabular}


Figure S3. X-ray analysis of compound 3g

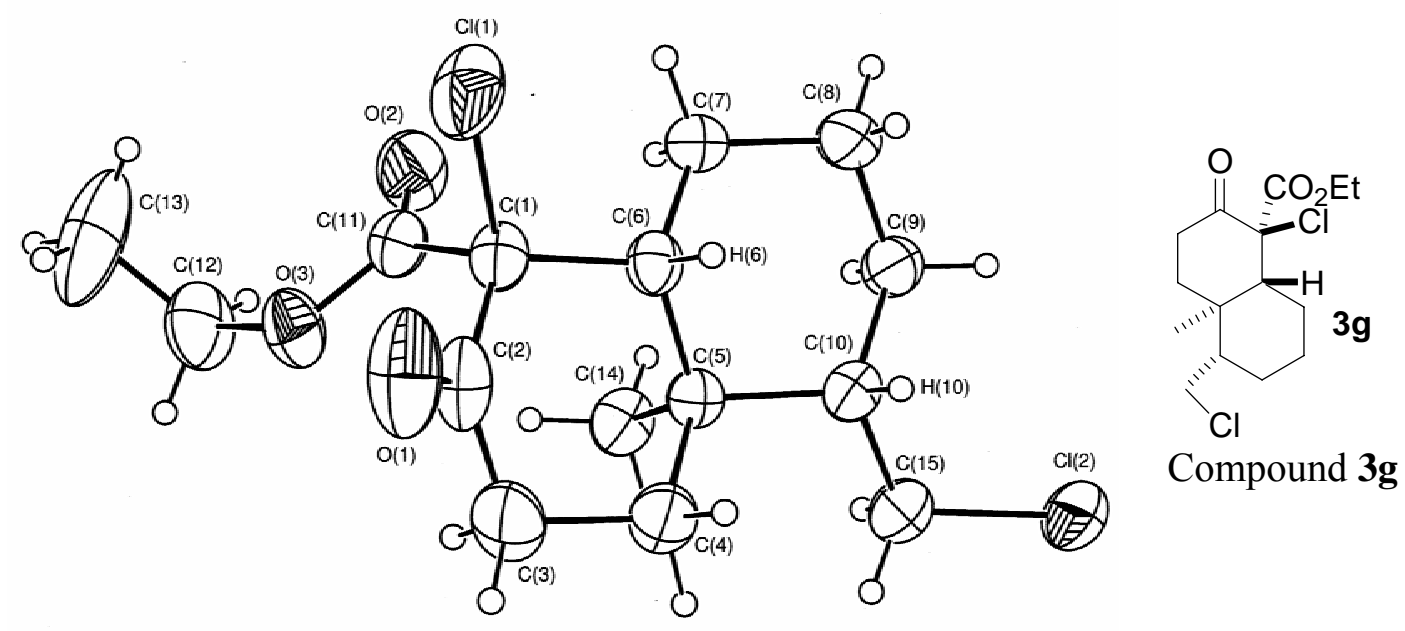


Table 1. Crystal data and structure refinement for mar793.

Identification code

Empirical formula

Formula weight

Temperature

Wavelength

Crystal system

Space group

Unit cell dimensions

Volume

Z

Density (calculated)

Absorption coefficient

$\mathrm{F}(000)$

Crystal size

Theta range for data collection

Index ranges

Reflections collected

Independent reflections

Completeness to theta $=25.15^{\circ}$

Absorption correction

Refinement method

Data / restraints / parameters

Goodness-of-fit on $\mathrm{F}^{2}$

Final R indices $[\mathrm{I}>2 \operatorname{sigma}(\mathrm{I})]$

$\mathrm{R}$ indices (all data)

Largest diff. peak and hole mar793

$\mathrm{C}_{15} \mathrm{H}_{22} \mathrm{Cl}_{2} \mathrm{O}_{3}$

321.23

253(2) K

$0.71073 \AA$

Triclinic

$\mathrm{P}-1(\# 2)$

$\mathrm{a}=8.154(2) \AA$

$=105.69(3)^{\circ}$

$\mathrm{b}=8.433(2) \AA$

$=96.24(3)^{\circ}$.

$\mathrm{c}=12.531(3) \AA$

$=94.71(3)^{\circ}$.
2

$1.303 \mathrm{Mg} / \mathrm{m}^{3}$

$0.400 \mathrm{~mm}^{-1}$

340

$0.5 \times 0.2 \times 0.1 \mathrm{~mm}^{3}$

1.70 to $25.15^{\circ}$.

$-8<=\mathrm{h}<=9,-9<=\mathrm{k}<=9,-13<=\mathrm{l}<=13$

2350

$1600[\mathrm{R}($ int $)=0.0331]$

$54.7 \%$

None

Full-matrix least-squares on $\mathrm{F}^{2}$

$1600 / 0 / 183$

1.020

$\mathrm{R} 1=0.0553, \mathrm{wR} 2=0.1640$

$\mathrm{R} 1=0.0756, \mathrm{wR} 2=0.1853$

0.274 and -0.337 e. $\AA^{-3}$ 
Table 2. Atomic coordinates $\left(\times 10^{4}\right)$ and equivalent isotropic displacement parameters $\left(\AA^{2} \times 10^{3}\right)$ for mar793. $U(e q)$ is defined as one third of the trace of the orthogonalized $U^{i j}$ tensor.

\begin{tabular}{|c|c|c|c|c|}
\hline & $\mathrm{x}$ & $\mathrm{y}$ & $\mathrm{z}$ & $\mathrm{U}(\mathrm{eq})$ \\
\hline $\mathrm{Cl}(1)$ & $8056(2)$ & $5488(2)$ & $4296(1)$ & $117(1)$ \\
\hline $\mathrm{Cl}(2)$ & $-575(2)$ & $2114(2)$ & $519(1)$ & $107(1)$ \\
\hline $\mathrm{O}(1)$ & $5919(6)$ & $7682(5)$ & $5448(3)$ & $127(2)$ \\
\hline $\mathrm{O}(2)$ & $8101(5)$ & $6825(4)$ & 2183(4) & $104(1)$ \\
\hline $\mathrm{O}(3)$ & $7380(4)$ & $8961(4)$ & $3457(3)$ & $87(1)$ \\
\hline $\mathrm{C}(1)$ & $6421(5)$ & $6287(5)$ & $3568(3)$ & $63(1)$ \\
\hline$C(2)$ & $5469(7)$ & $7345(6)$ & $4455(4)$ & $83(2)$ \\
\hline$C(3)$ & $3899(8)$ & $7844(6)$ & $3976(5)$ & $102(2)$ \\
\hline$C(4)$ & $2763(6)$ & $6334(6)$ & $3213(4)$ & $80(1)$ \\
\hline$C(5)$ & $3617(5)$ & $5305(4)$ & $2253(3)$ & $55(1)$ \\
\hline$C(6)$ & $5211(5)$ & $4802(5)$ & 2783(3) & $58(1)$ \\
\hline$C(7)$ & $6066(6)$ & $3612(5)$ & 1919(4) & $79(1)$ \\
\hline$C(8)$ & 4919(6) & $2000(6)$ & $1381(5)$ & $97(2)$ \\
\hline$C(9)$ & $3335(6)$ & $2374(5)$ & $818(4)$ & $78(1)$ \\
\hline$C(10)$ & $2462(5)$ & $3636(5)$ & $1635(4)$ & $63(1)$ \\
\hline$C(14)$ & $3944(6)$ & $6330(5)$ & $1434(4)$ & $73(1)$ \\
\hline$C(11)$ & $7404(6)$ & $7359(6)$ & $2976(4)$ & $68(1)$ \\
\hline$C(12)$ & $8255(9)$ & $10126(7)$ & $2948(5)$ & $109(2)$ \\
\hline$C(13)$ & $9912(11)$ & $10577(11)$ & $3456(6)$ & $192(4)$ \\
\hline$C(15)$ & $867(6)$ & $3939(6)$ & $1042(5)$ & $90(2)$ \\
\hline
\end{tabular}


Table 3. Bond lengths $[\AA]$ and angles $\left[^{\circ}\right]$ for mar793.

\begin{tabular}{|c|c|c|c|}
\hline $\mathrm{Cl}(1)-\mathrm{C}(1)$ & $1.811(4)$ & $C(6)-C(5)-C(14)$ & $113.2(3)$ \\
\hline $\mathrm{Cl}(2)-\mathrm{C}(15)$ & $1.781(5)$ & $\mathrm{C}(6)-\mathrm{C}(5)-\mathrm{C}(4)$ & 107.8(3) \\
\hline $\mathrm{O}(1)-\mathrm{C}(2)$ & $1.207(5)$ & $C(14)-C(5)-C(4)$ & 109.3(3) \\
\hline $\mathrm{O}(2)-\mathrm{C}(11)$ & $1.199(5)$ & $C(6)-C(5)-C(10)$ & 106.3(3) \\
\hline $\mathrm{O}(3)-\mathrm{C}(11)$ & $1.325(5)$ & $\mathrm{C}(14)-\mathrm{C}(5)-\mathrm{C}(10)$ & $110.9(3)$ \\
\hline $\mathrm{O}(3)-\mathrm{C}(12)$ & $1.485(6)$ & $C(4)-C(5)-C(10)$ & 109.2(3) \\
\hline$C(1)-C(11)$ & $1.543(6)$ & $C(7)-C(6)-C(5)$ & $112.4(3)$ \\
\hline $\mathrm{C}(1)-\mathrm{C}(2)$ & $1.549(7)$ & $\mathrm{C}(7)-\mathrm{C}(6)-\mathrm{C}(1)$ & 113.3(3) \\
\hline$C(1)-C(6)$ & $1.559(5)$ & $\mathrm{C}(5)-\mathrm{C}(6)-\mathrm{C}(1)$ & $114.2(3)$ \\
\hline$C(2)-C(3)$ & $1.495(8)$ & $C(8)-C(7)-C(6)$ & $110.0(4)$ \\
\hline$C(3)-C(4)$ & $1.532(7)$ & $\mathrm{C}(9)-\mathrm{C}(8)-\mathrm{C}(7)$ & $109.5(4)$ \\
\hline$C(4)-C(5)$ & $1.550(5)$ & $\mathrm{C}(8)-\mathrm{C}(9)-\mathrm{C}(10)$ & $112.0(4)$ \\
\hline$C(5)-C(6)$ & $1.538(5)$ & $\mathrm{C}(15)-\mathrm{C}(10)-\mathrm{C}(9)$ & $110.1(4)$ \\
\hline$C(5)-C(14)$ & $1.540(5)$ & $C(15)-C(10)-C(5)$ & $112.1(3)$ \\
\hline$C(5)-C(10)$ & $1.582(6)$ & $\mathrm{C}(9)-\mathrm{C}(10)-\mathrm{C}(5)$ & $113.2(3)$ \\
\hline$C(6)-C(7)$ & $1.536(5)$ & $\mathrm{O}(2)-\mathrm{C}(11)-\mathrm{O}(3)$ & $123.9(4)$ \\
\hline$C(7)-C(8)$ & $1.529(7)$ & $\mathrm{O}(2)-\mathrm{C}(11)-\mathrm{C}(1)$ & $124.9(4)$ \\
\hline $\mathrm{C}(8)-\mathrm{C}(9)$ & $1.501(7)$ & $\mathrm{O}(3)-\mathrm{C}(11)-\mathrm{C}(1)$ & $111.3(4)$ \\
\hline$C(9)-C(10)$ & $1.543(6)$ & $\mathrm{C}(13)-\mathrm{C}(12)-\mathrm{O}(3)$ & $110.8(5)$ \\
\hline$C(10)-C(15)$ & $1.502(6)$ & $\mathrm{C}(10)-\mathrm{C}(15)-\mathrm{Cl}(2)$ & $112.7(3)$ \\
\hline $\mathrm{C}(12)-\mathrm{C}(13)$ & $1.405(9)$ & & \\
\hline $\mathrm{C}(11)-\mathrm{O}(3)-\mathrm{C}(12)$ & $116.5(4)$ & & \\
\hline$C(11)-C(1)-C(2)$ & $111.9(3)$ & & \\
\hline $\mathrm{C}(11)-\mathrm{C}(1)-\mathrm{C}(6)$ & $115.7(3)$ & & \\
\hline $\mathrm{C}(2)-\mathrm{C}(1)-\mathrm{C}(6)$ & 109.4(3) & & \\
\hline $\mathrm{C}(11)-\mathrm{C}(1)-\mathrm{Cl}(1)$ & $102.4(3)$ & & \\
\hline $\mathrm{C}(2)-\mathrm{C}(1)-\mathrm{Cl}(1)$ & 107.9(3) & & \\
\hline $\mathrm{C}(6)-\mathrm{C}(1)-\mathrm{Cl}(1)$ & $108.9(3)$ & & \\
\hline $\mathrm{O}(1)-\mathrm{C}(2)-\mathrm{C}(3)$ & $122.8(5)$ & & \\
\hline $\mathrm{O}(1)-\mathrm{C}(2)-\mathrm{C}(1)$ & $123.0(6)$ & & \\
\hline$C(3)-C(2)-C(1)$ & $114.1(4)$ & & \\
\hline $\mathrm{C}(2)-\mathrm{C}(3)-\mathrm{C}(4)$ & $111.3(4)$ & & \\
\hline$C(3)-C(4)-C(5)$ & $113.1(4)$ & & \\
\hline
\end{tabular}


Symmetry transformations used to generate equivalent atoms: 
Table 4. Anisotropic displacement parameters $\left(\AA^{2} \times 10^{3}\right)$ for mar793. The anisotropic displacement factor exponent takes the form: $-2 \quad{ }^{2}\left[\mathrm{~h}^{2} \mathrm{a}^{* 2} \mathrm{U}^{11}+\ldots+2 \mathrm{~h} \mathrm{k} \mathrm{a}^{*} \mathrm{~b}^{*} \mathrm{U}^{12}\right]$

\begin{tabular}{|c|c|c|c|c|c|c|}
\hline & $\mathrm{U}^{11}$ & $\mathrm{U}^{22}$ & $\mathrm{U}^{33}$ & $\mathrm{U}^{23}$ & $\mathrm{U}^{13}$ & $\mathrm{U}^{12}$ \\
\hline $\mathrm{Cl}(1)$ & $104(1)$ & 111(1) & $130(1)$ & $62(1)$ & $-51(1)$ & $-20(1)$ \\
\hline $\mathrm{Cl}(2)$ & $61(1)$ & $96(1)$ & $137(1)$ & $3(1)$ & $-11(1)$ & $-2(1)$ \\
\hline $\mathrm{O}(1)$ & 171(4) & 133(3) & $51(2)$ & $10(2)$ & $3(2)$ & $-60(3)$ \\
\hline $\mathrm{O}(2)$ & $99(3)$ & $94(3)$ & $122(3)$ & $27(2)$ & $52(3)$ & $-5(2)$ \\
\hline $\mathrm{O}(3)$ & $106(3)$ & $60(2)$ & $95(2)$ & $26(2)$ & $20(2)$ & $-8(2)$ \\
\hline $\mathrm{C}(1)$ & $67(3)$ & $63(3)$ & $59(3)$ & $25(2)$ & $-5(2)$ & $-4(2)$ \\
\hline$C(2)$ & $99(4)$ & $76(3)$ & $60(3)$ & $12(3)$ & $10(3)$ & $-32(3)$ \\
\hline$C(3)$ & $104(4)$ & $91(4)$ & $93(4)$ & $-14(3)$ & $48(4)$ & $2(3)$ \\
\hline$C(4)$ & $74(3)$ & $83(3)$ & $72(3)$ & $4(3)$ & $22(3)$ & $3(2)$ \\
\hline$C(5)$ & $58(3)$ & $57(2)$ & $52(2)$ & $17(2)$ & $10(2)$ & $4(2)$ \\
\hline$C(6)$ & $62(3)$ & $57(2)$ & $57(2)$ & $21(2)$ & $3(2)$ & $-1(2)$ \\
\hline$C(7)$ & $61(3)$ & $65(3)$ & 103(3) & $10(3)$ & $4(3)$ & $13(2)$ \\
\hline$C(8)$ & $68(3)$ & $66(3)$ & 133(4) & $-7(3)$ & $-3(3)$ & $15(3)$ \\
\hline$C(9)$ & $64(3)$ & $71(3)$ & $81(3)$ & $-6(2)$ & $6(3)$ & $7(2)$ \\
\hline$C(10)$ & $55(3)$ & $67(3)$ & $67(3)$ & $20(2)$ & $7(2)$ & $2(2)$ \\
\hline$C(14)$ & $73(3)$ & $82(3)$ & $70(3)$ & $37(2)$ & $6(3)$ & $2(2)$ \\
\hline $\mathrm{C}(11)$ & $64(3)$ & $69(3)$ & $71(3)$ & $22(3)$ & $9(3)$ & $-4(2)$ \\
\hline$C(12)$ & $127(5)$ & $83(4)$ & $124(4)$ & $53(3)$ & $11(4)$ & $-15(3)$ \\
\hline $\mathrm{C}(13)$ & $160(8)$ & $225(9)$ & 183(7) & $120(7)$ & $-57(7)$ & $-118(7)$ \\
\hline$C(15)$ & $60(3)$ & $84(3)$ & $115(4)$ & $18(3)$ & $-12(3)$ & $5(2)$ \\
\hline
\end{tabular}


Table 5. Hydrogen coordinates ( $\left.\mathrm{x} 10^{4}\right)$ and isotropic displacement parameters $\left(\AA^{2} \mathrm{x} 10^{3}\right)$ for $\operatorname{mar} 793$.

\begin{tabular}{|c|c|c|c|c|}
\hline & $\mathrm{x}$ & $\mathrm{y}$ & $\mathrm{z}$ & $\mathrm{U}(\mathrm{eq})$ \\
\hline $\mathrm{H}(3 \mathrm{~A})$ & 4165 & 8614 & 3552 & 122 \\
\hline $\mathrm{H}(3 \mathrm{~B})$ & 3320 & 8405 & 4580 & 122 \\
\hline $\mathrm{H}(4 \mathrm{~A})$ & 1783 & 6709 & 2892 & 96 \\
\hline $\mathrm{H}(4 \mathrm{~B})$ & 2404 & 5628 & 3659 & 96 \\
\hline $\mathrm{H}(6)$ & 4839 & 4153 & 3272 & 70 \\
\hline $\mathrm{H}(7 \mathrm{~A})$ & 6339 & 4128 & 1347 & 95 \\
\hline $\mathrm{H}(7 \mathrm{~B})$ & 7091 & 3373 & 2282 & 95 \\
\hline $\mathrm{H}(8 \mathrm{~A})$ & 5463 & 1251 & 835 & 117 \\
\hline $\mathrm{H}(8 \mathrm{~B})$ & 4675 & 1463 & 1949 & 117 \\
\hline $\mathrm{H}(9 \mathrm{~A})$ & 2592 & 1354 & 507 & 93 \\
\hline $\mathrm{H}(9 \mathrm{~B})$ & 3579 & 2812 & 205 & 93 \\
\hline $\mathrm{H}(10)$ & 2166 & 3122 & 2212 & 76 \\
\hline $\mathrm{H}(14 \mathrm{~A})$ & 4713 & 7293 & 1812 & 109 \\
\hline $\mathrm{H}(14 \mathrm{~B})$ & 2919 & 6668 & 1168 & 109 \\
\hline $\mathrm{H}(14 \mathrm{C})$ & 4404 & 5667 & 813 & 109 \\
\hline $\mathrm{H}(12 \mathrm{~A})$ & 8229 & 9606 & 2154 & 131 \\
\hline $\mathrm{H}(12 \mathrm{~B})$ & 7689 & 11112 & 3035 & 131 \\
\hline $\mathrm{H}(13 \mathrm{~A})$ & 9939 & 11041 & 4248 & 287 \\
\hline $\mathrm{H}(13 \mathrm{~B})$ & 10433 & 11385 & 3150 & 287 \\
\hline $\mathrm{H}(13 \mathrm{C})$ & 10496 & 9614 & 3318 & 287 \\
\hline $\mathrm{H}(15 \mathrm{~A})$ & 1110 & 4366 & 424 & 108 \\
\hline $\mathrm{H}(15 \mathrm{~B})$ & 364 & 4776 & 1556 & 108 \\
\hline
\end{tabular}




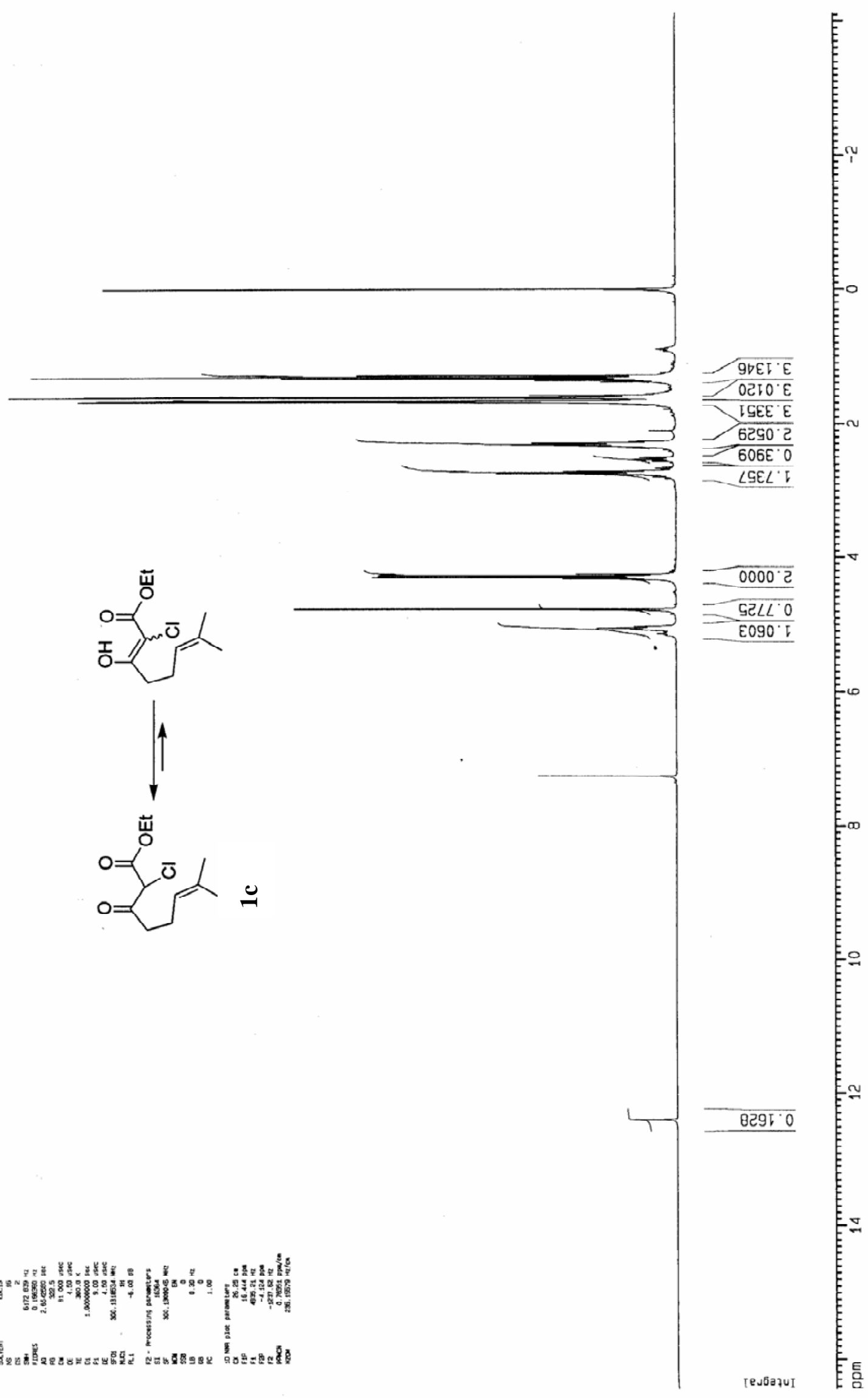




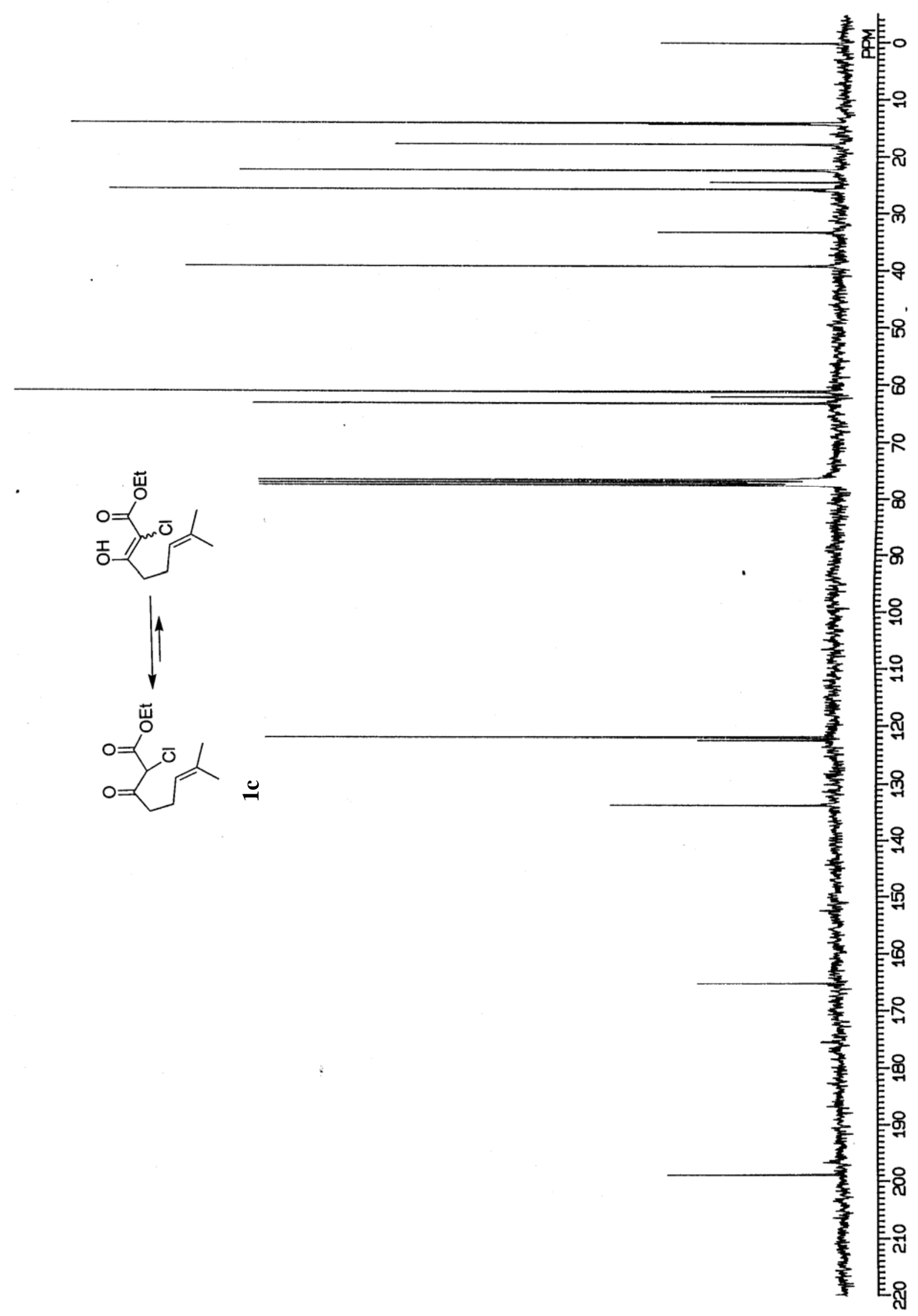




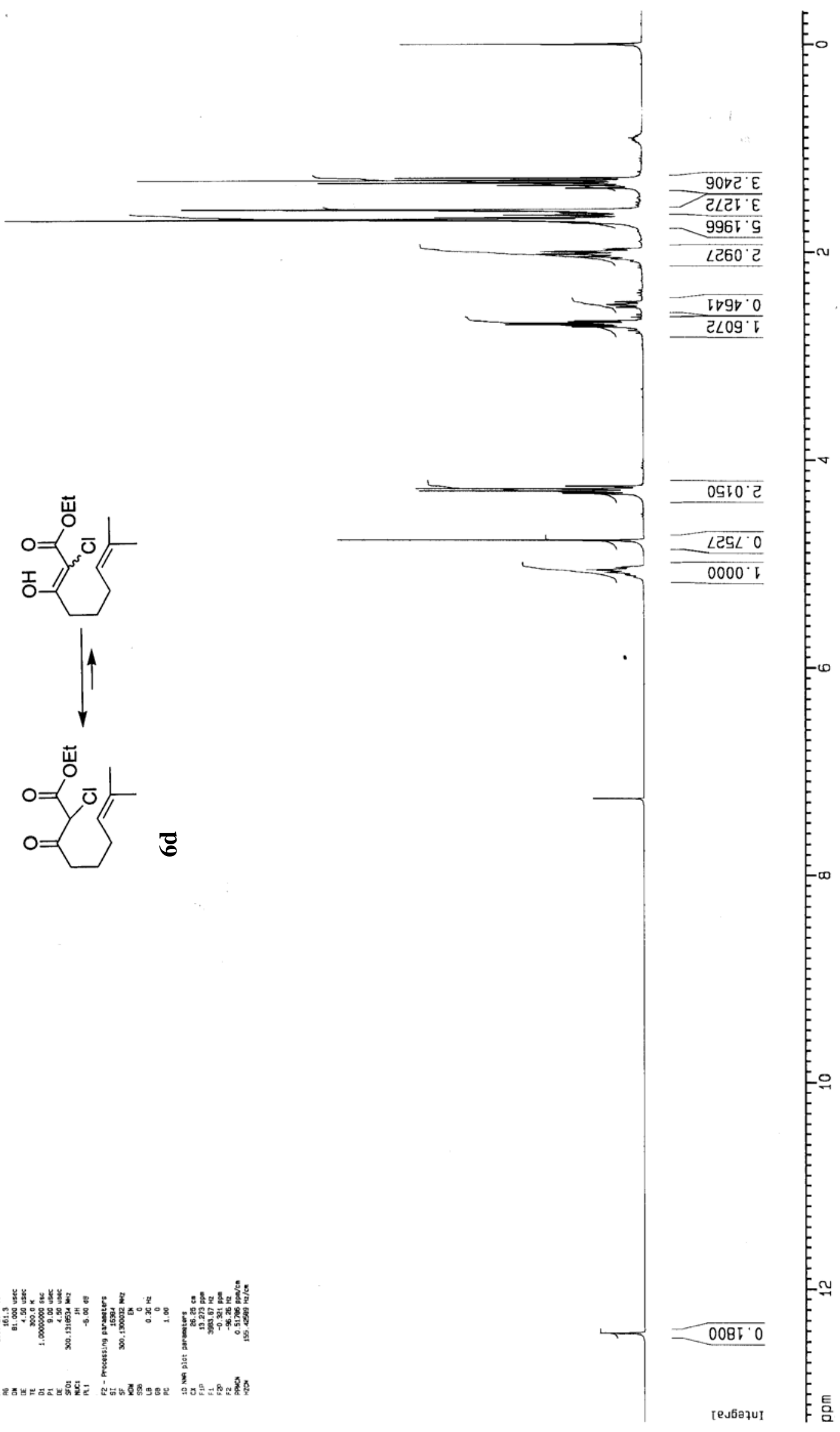


696 EI

$62 \angle{ }^{\prime} \angle T$

$899^{\circ} \varepsilon 己$

gटL 92

$690^{\circ} \mathrm{L}$

टBE $8 E$
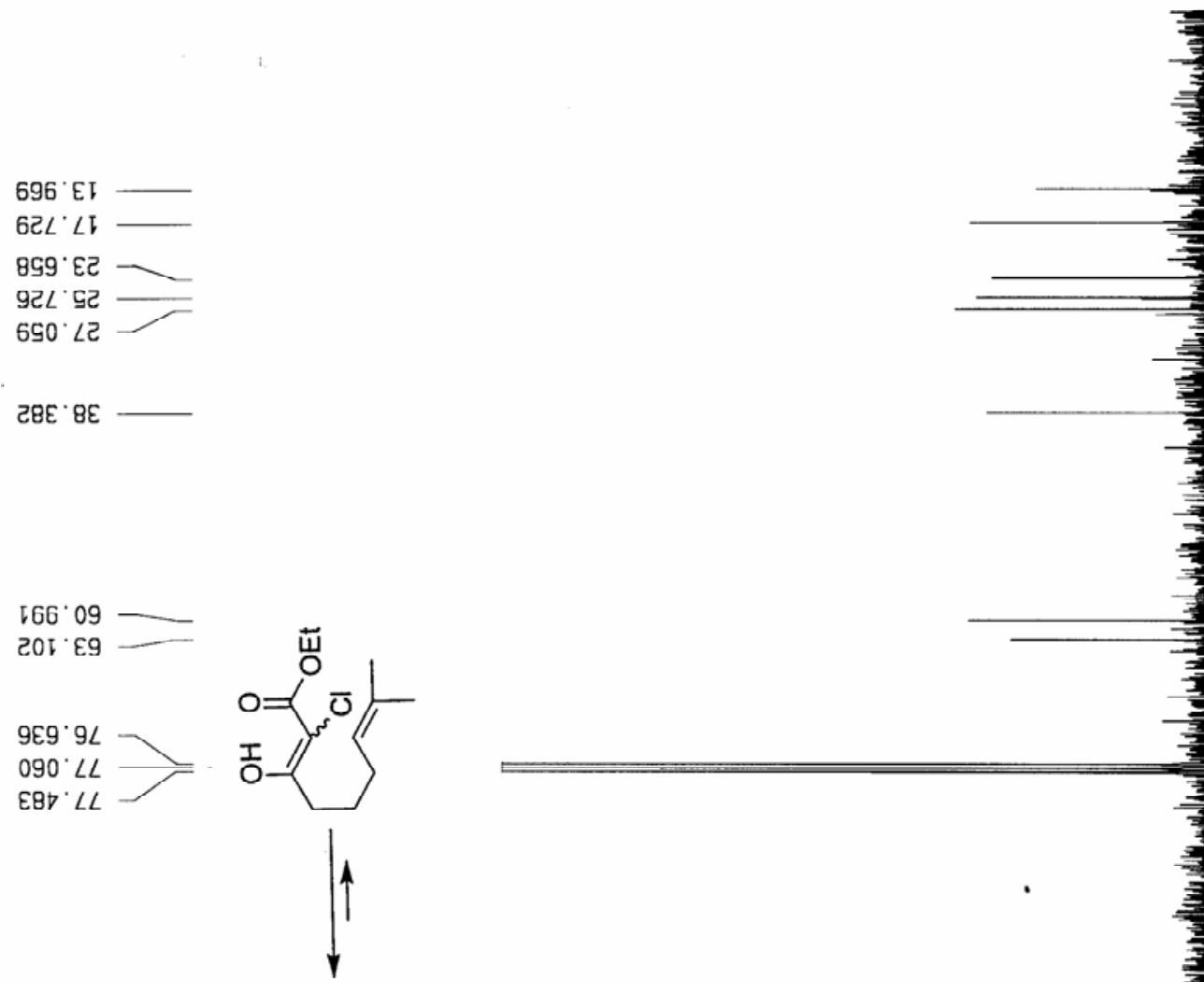

$\downarrow$

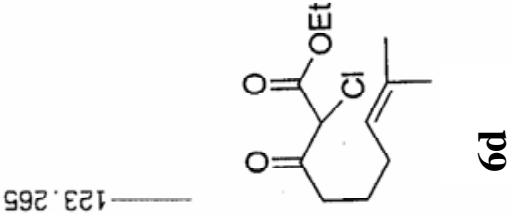

छ

G98. टEI-

टนเ 991
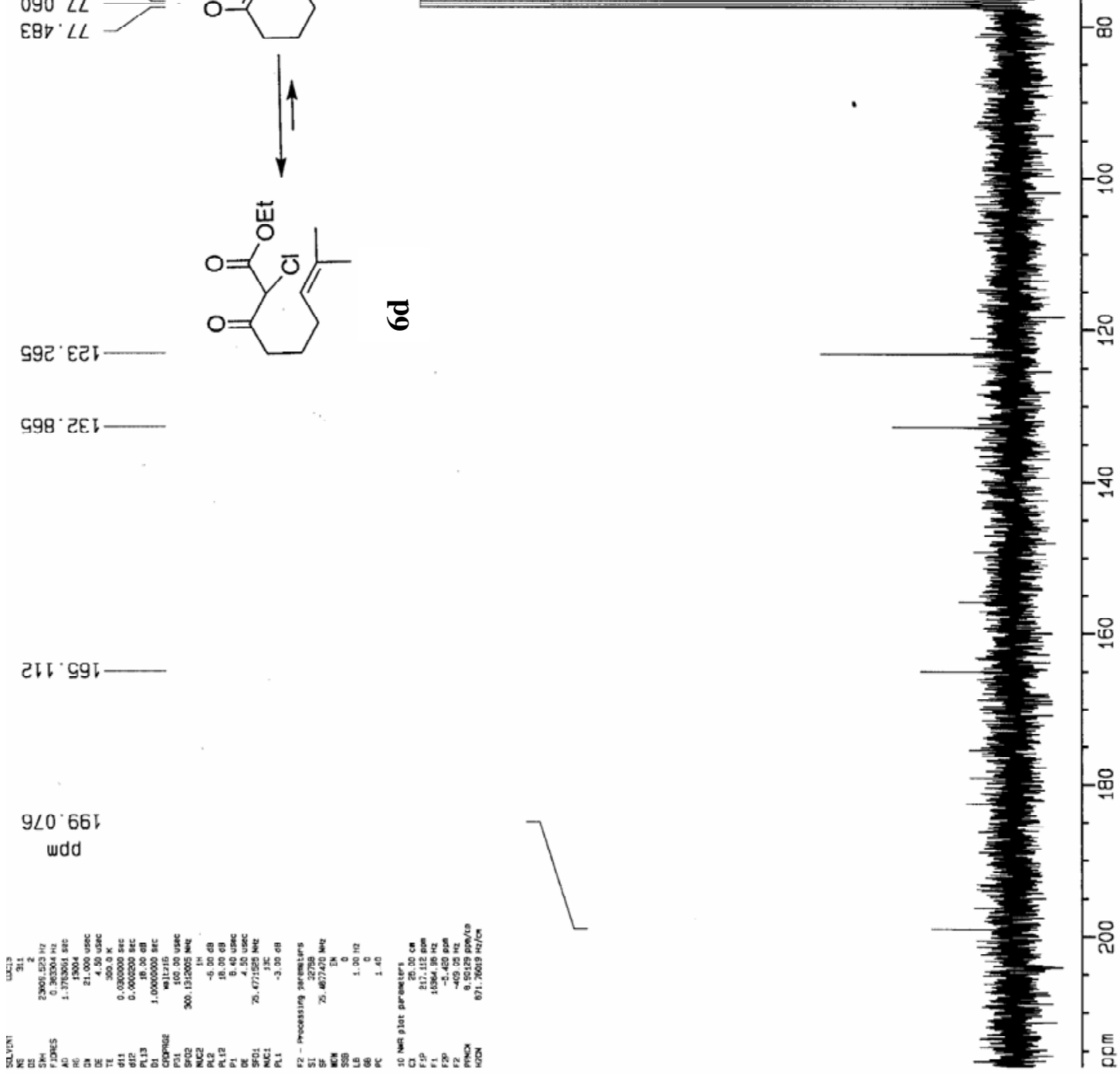


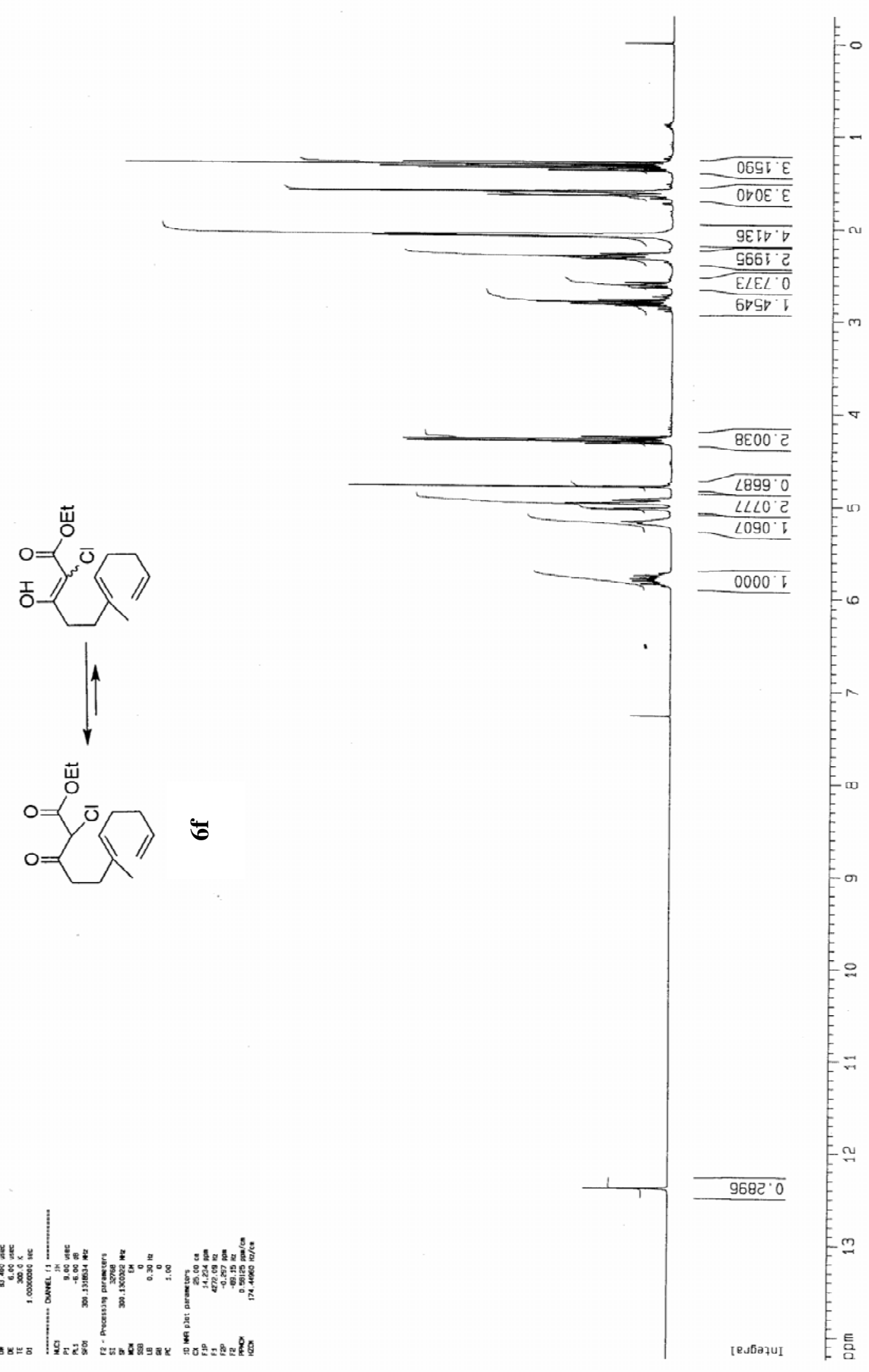



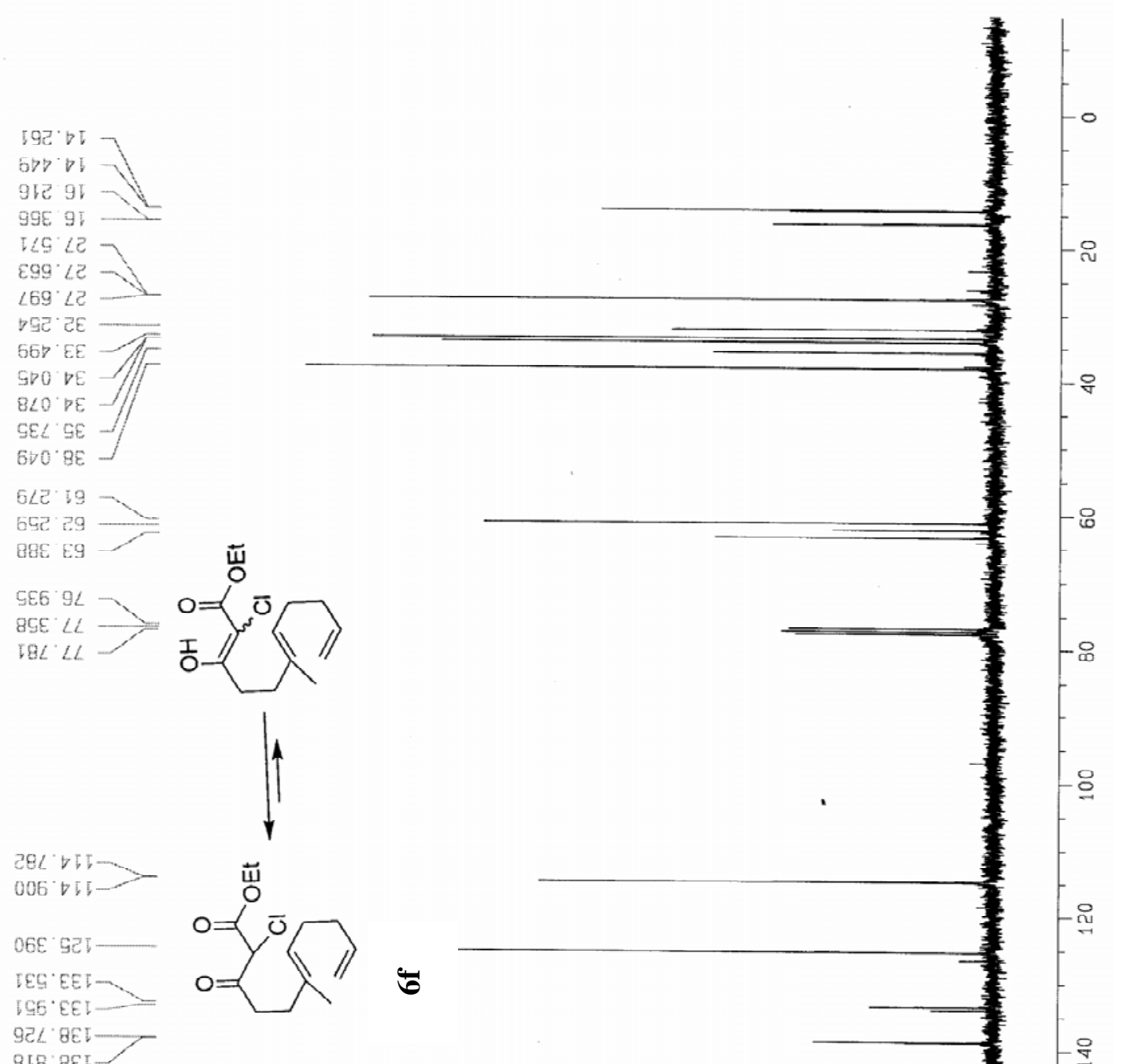

8LB BE

$\bar{\sigma}$

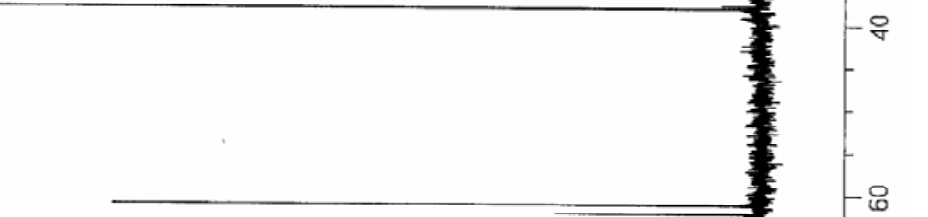

¿GE G96-_-

$199^{\circ} 9 \angle \mathrm{L}$

$568^{\circ} 865$

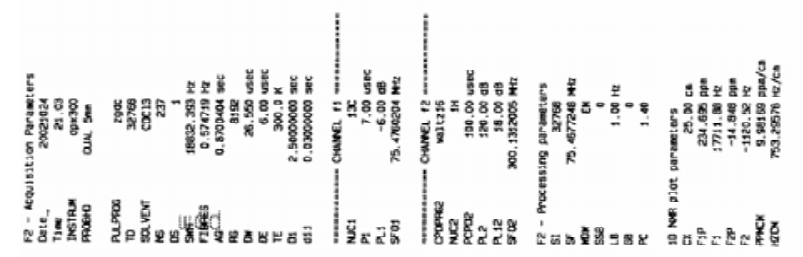




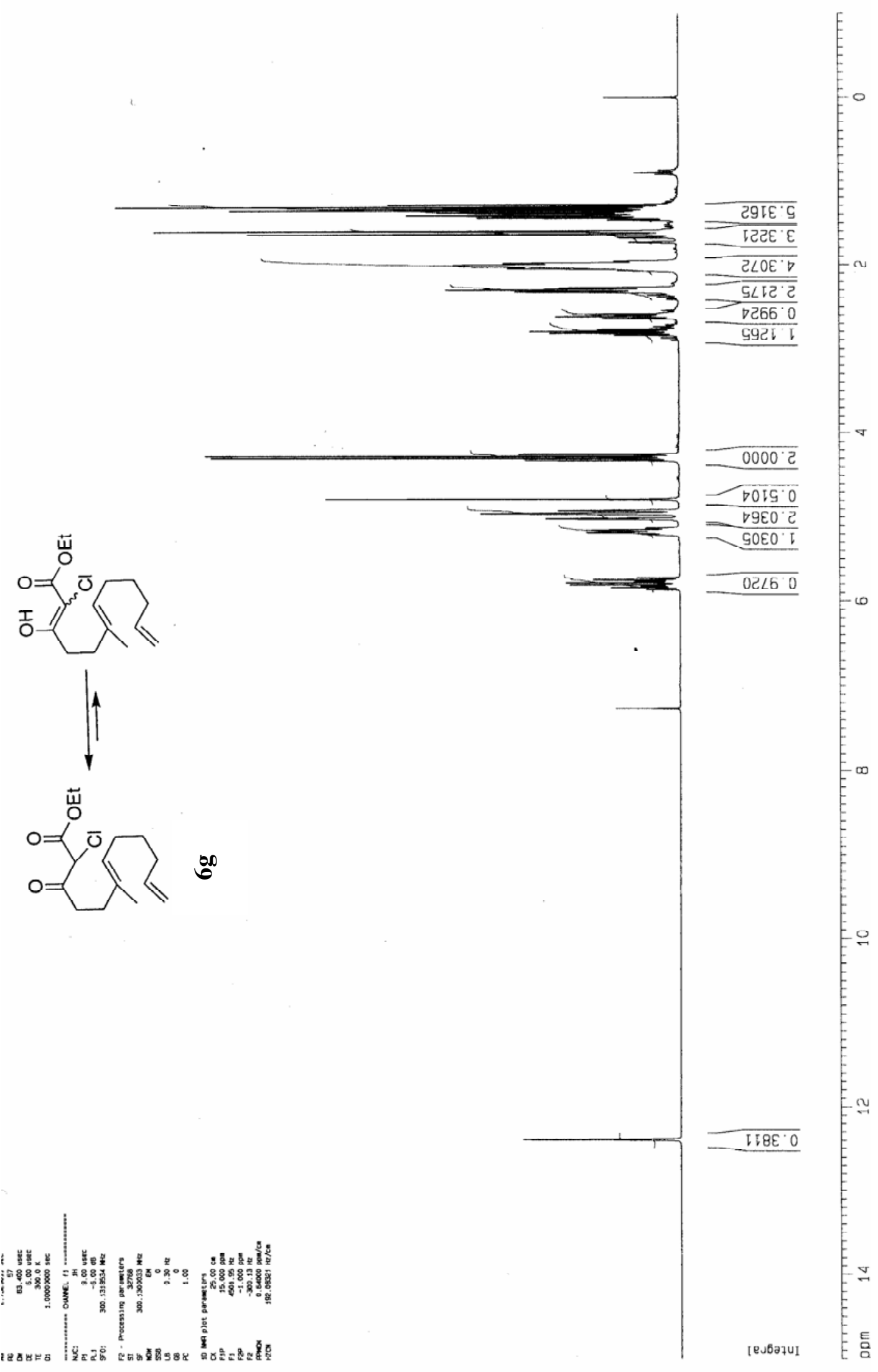



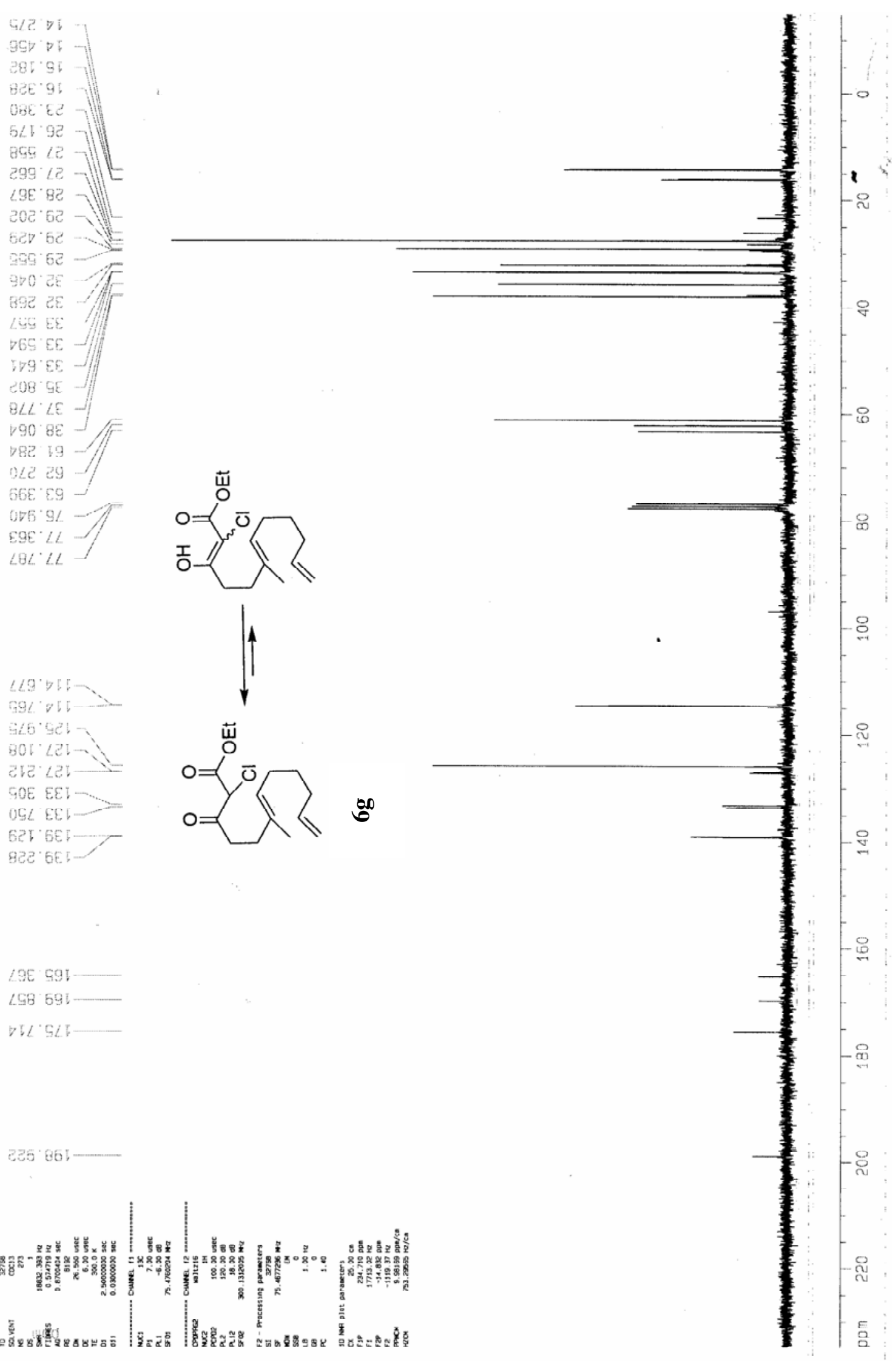


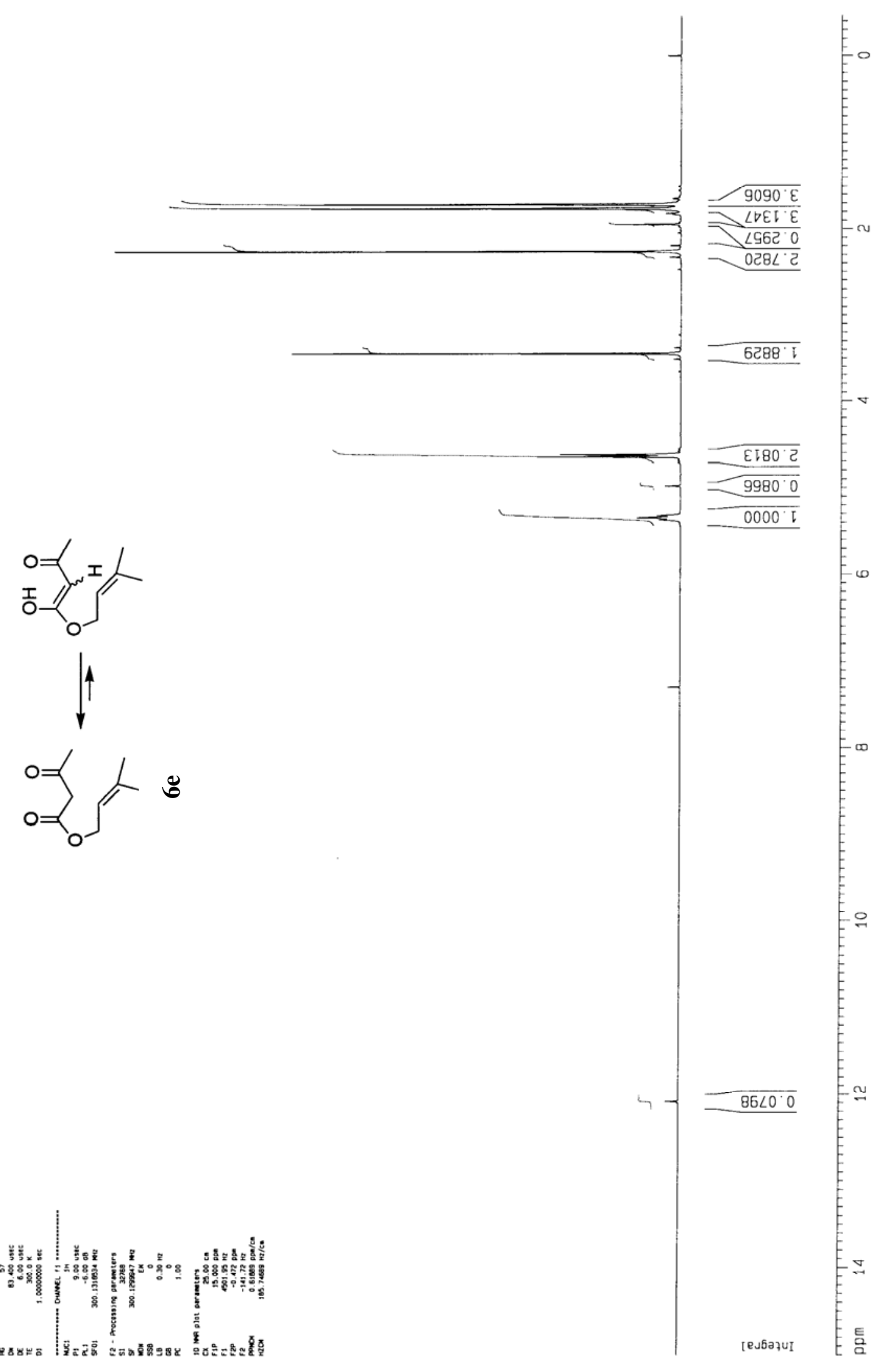



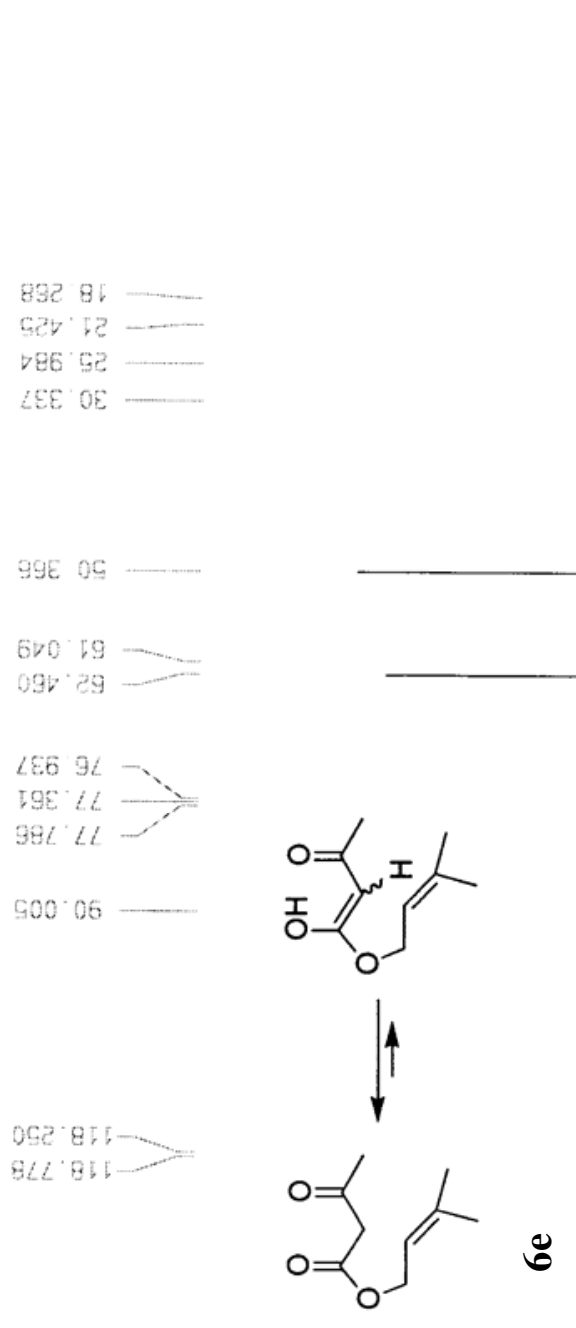

en: $00:-$

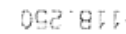

Q $\angle 2$ Q

$206 / 95$
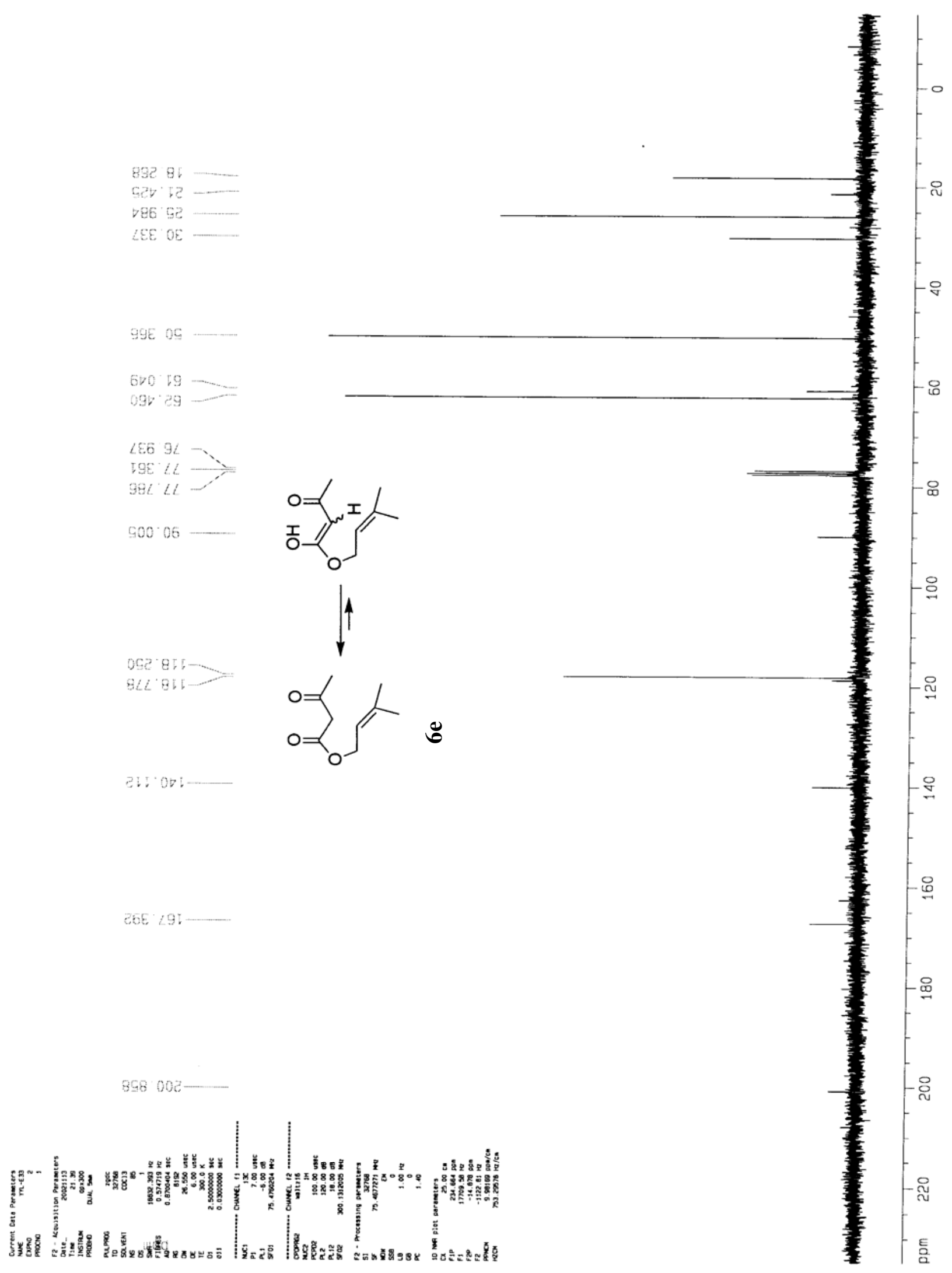


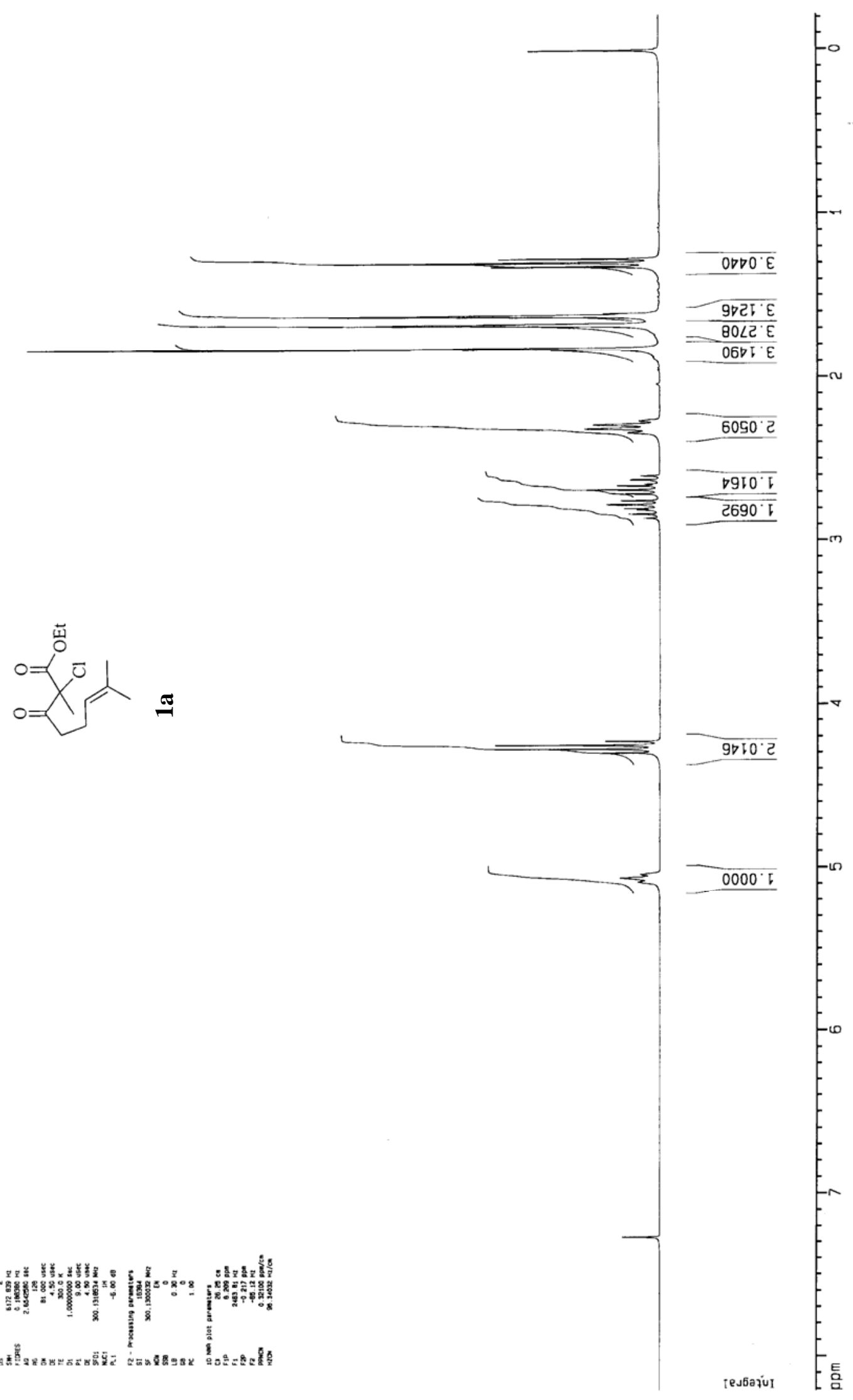


$100 \%$
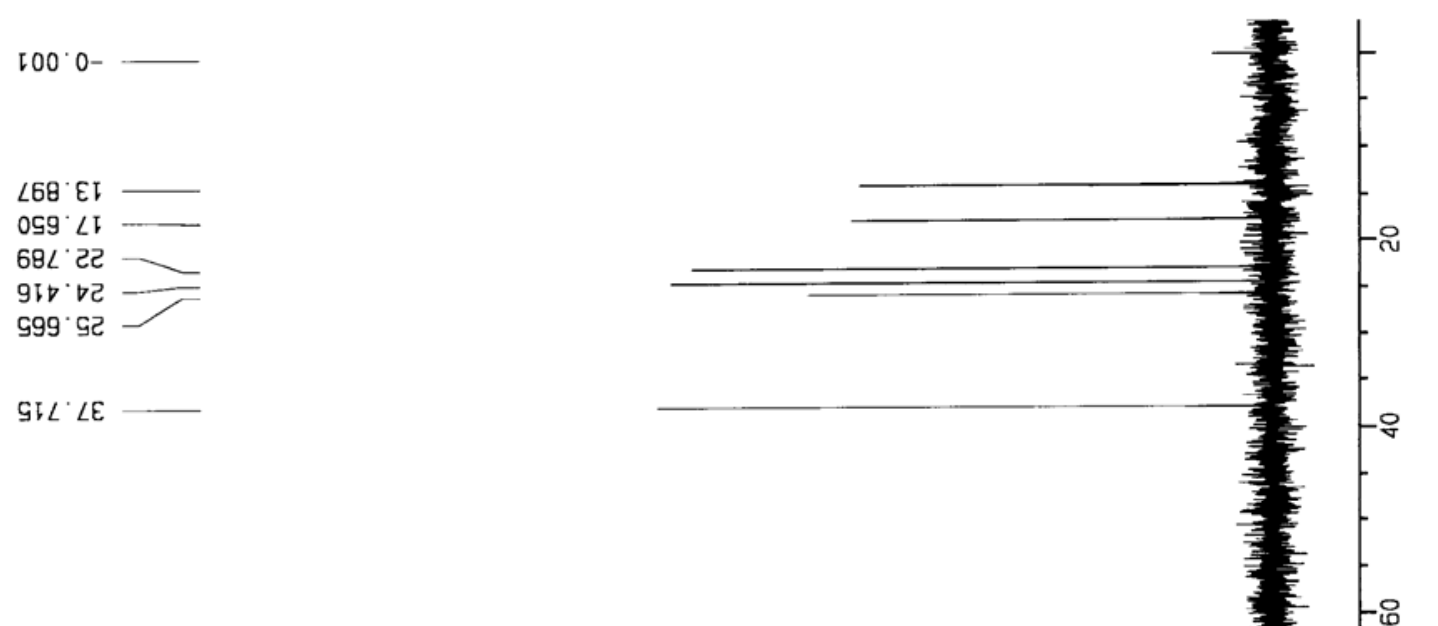

966'29

$568 \cdot 02$

$\angle I 9.9 \angle$

$O \forall 0^{\circ} \angle L$

$\checkmark 9 \sigma^{\circ} L L$

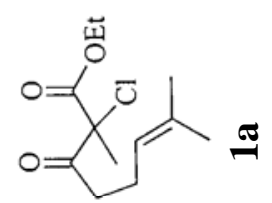

GL' ट己เ -

6GI EE -
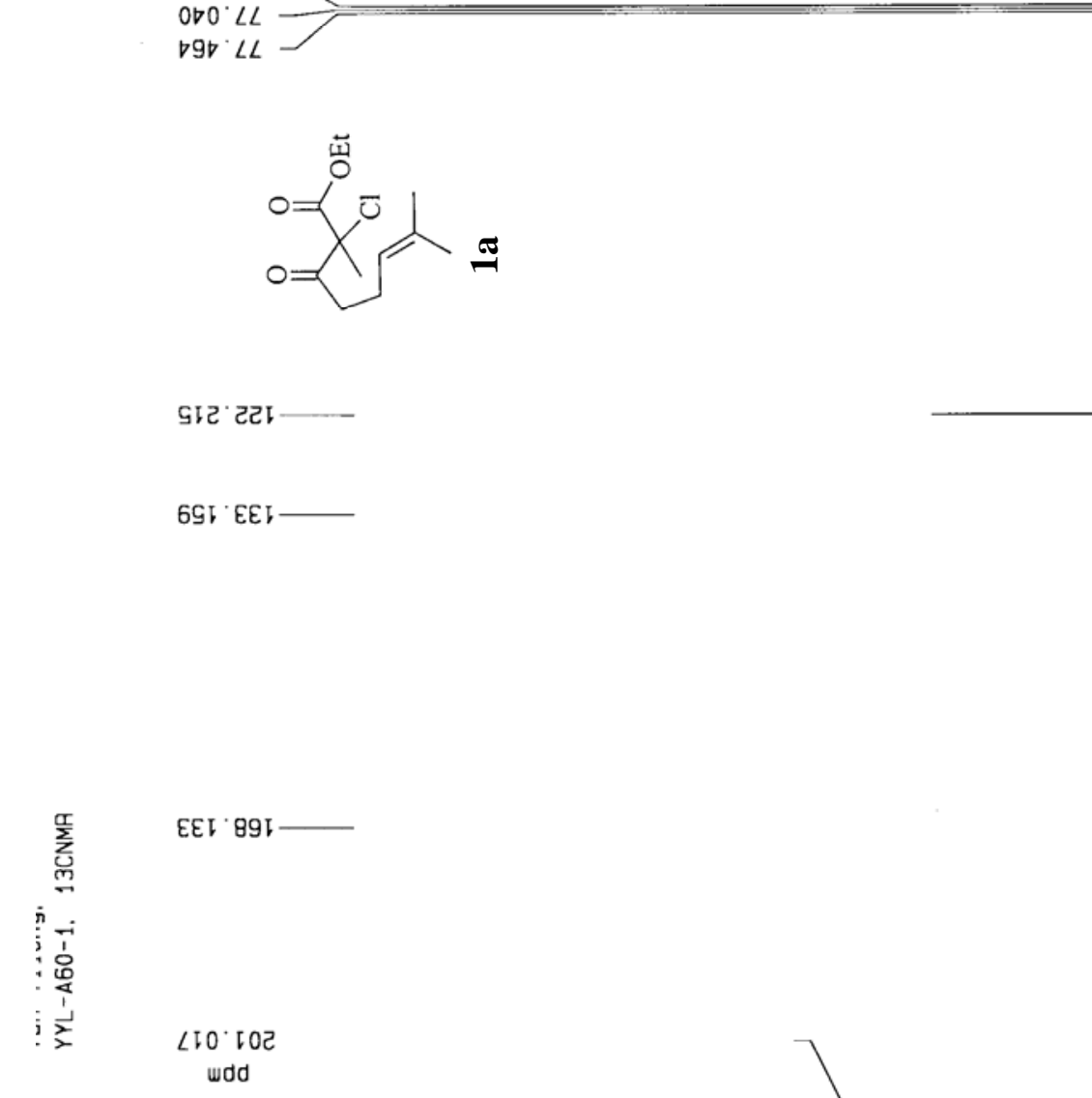

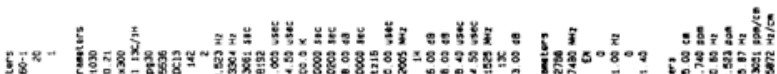

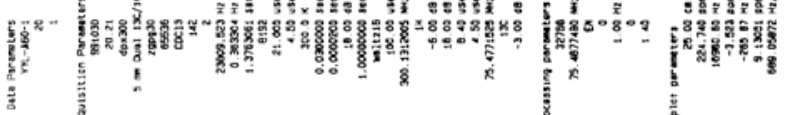

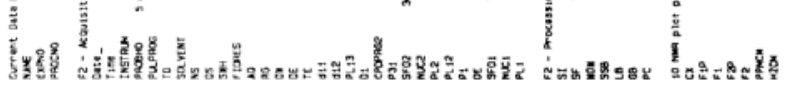

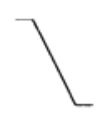



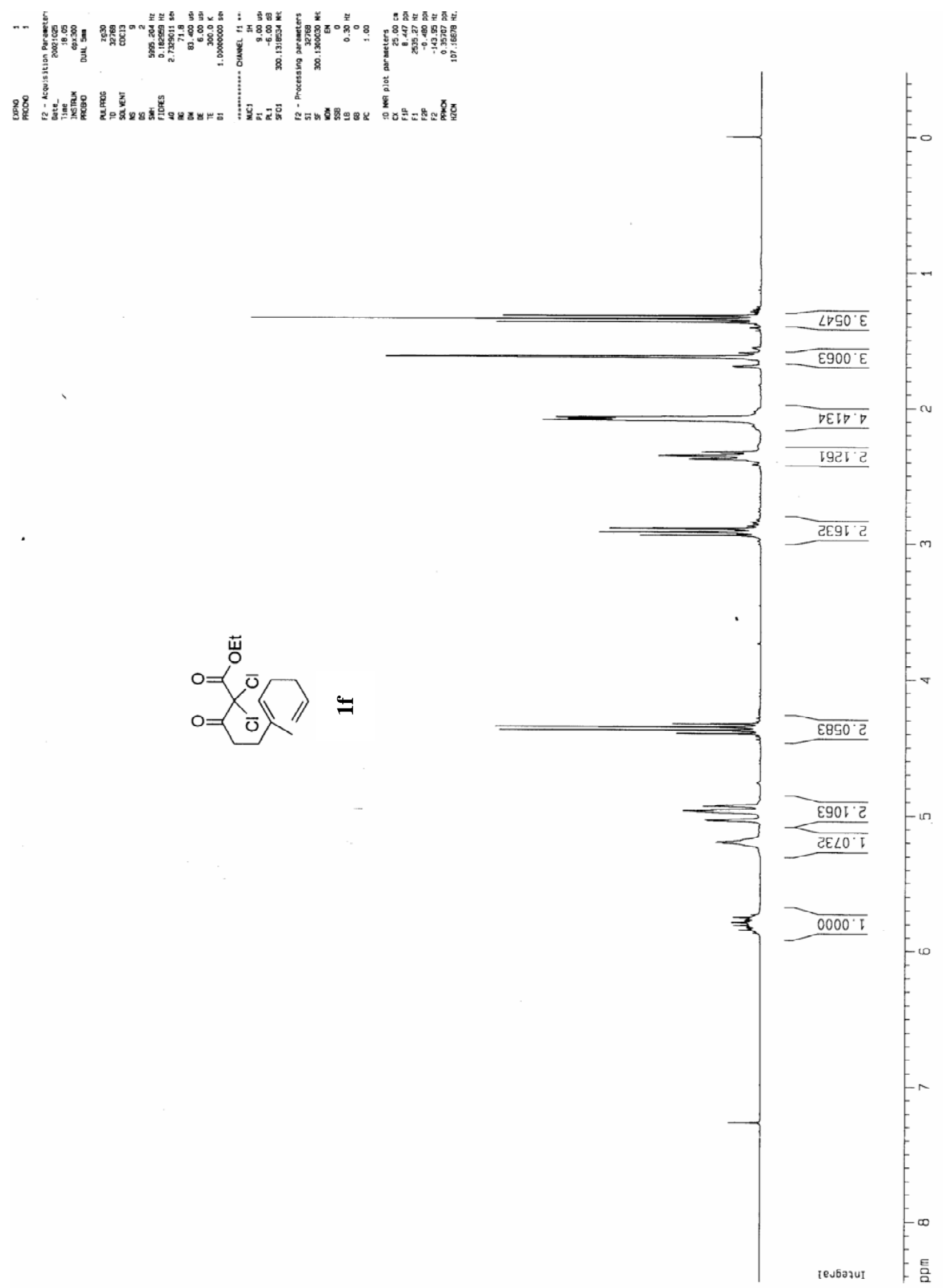


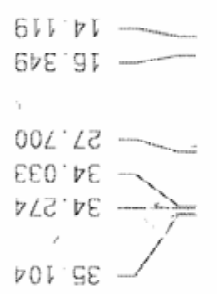<smiles>C=CCCC1(C(=O)[O-])CCC(=O)CC1=O</smiles>

$926 \circ I-$
$E \angle 0^{\circ} \mathrm{GI}$

$269^{\circ} \mathrm{GटI- \cdots -m-}$

GEE 'EEI-

हटL'BEL

8GL'E9I-

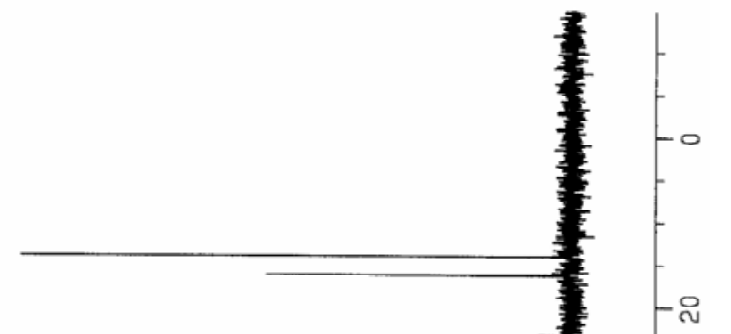

५06 $\triangleright 9$

EE6 $9 L$

$\angle G E \angle L$

65228

890 D6I -

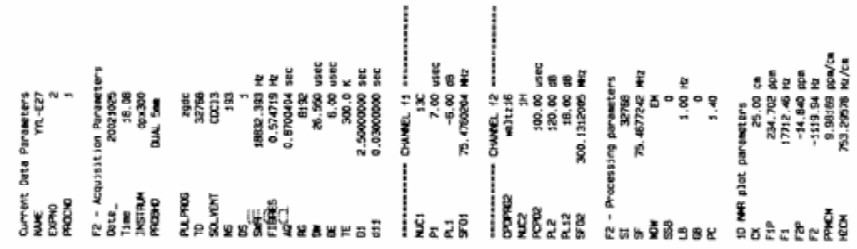




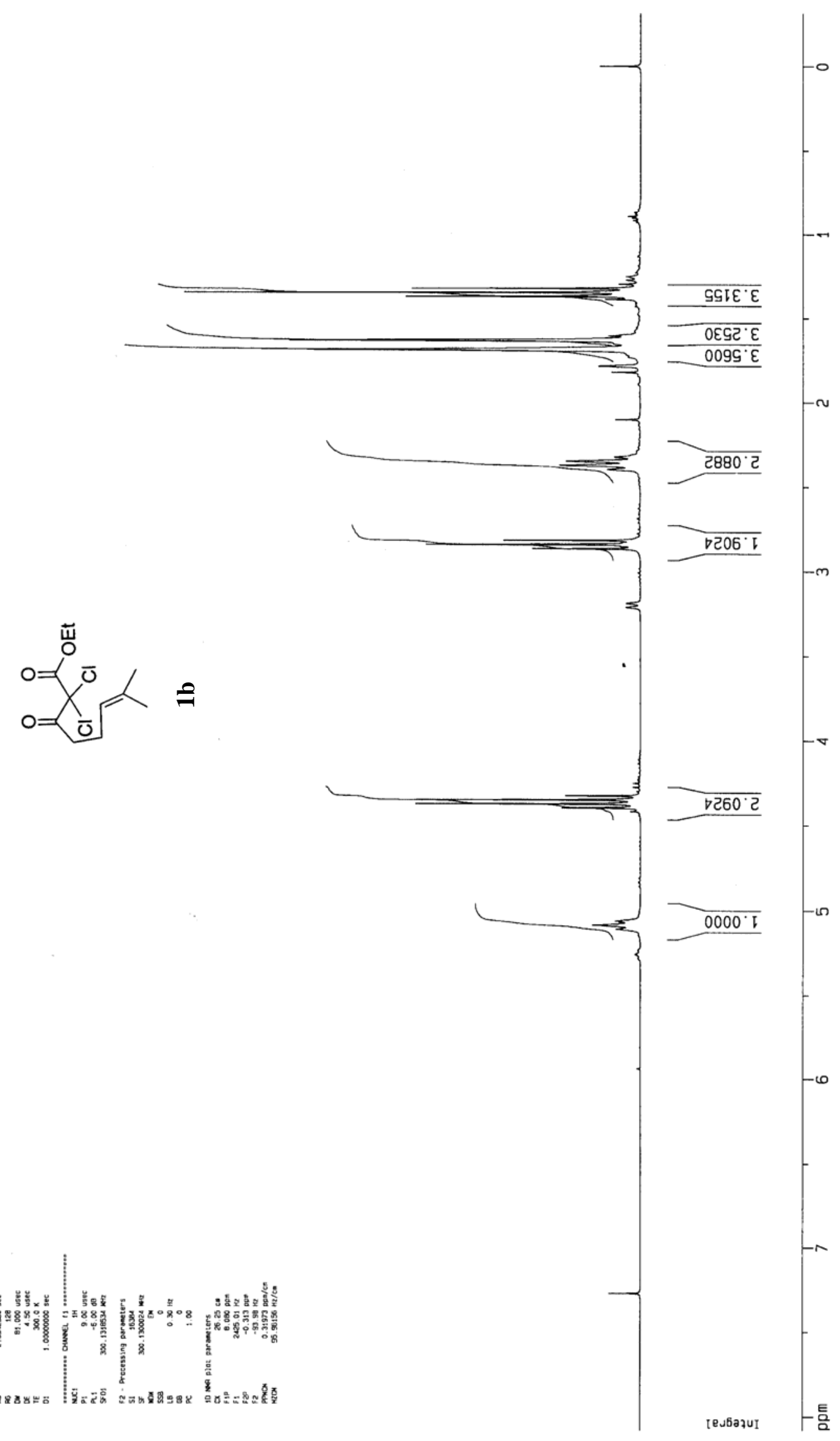


GOปจ

$\nabla \angle 6^{\circ} \angle L$

IOE $E 己$

896 '

6 เ $9 \varepsilon$

$\angle 06^{\circ} \nabla 9$

OE6 $9 L$

$\checkmark G E^{\circ} \angle L$

$\angle L L^{\circ} \angle L$

$62{ }^{\circ} 28$

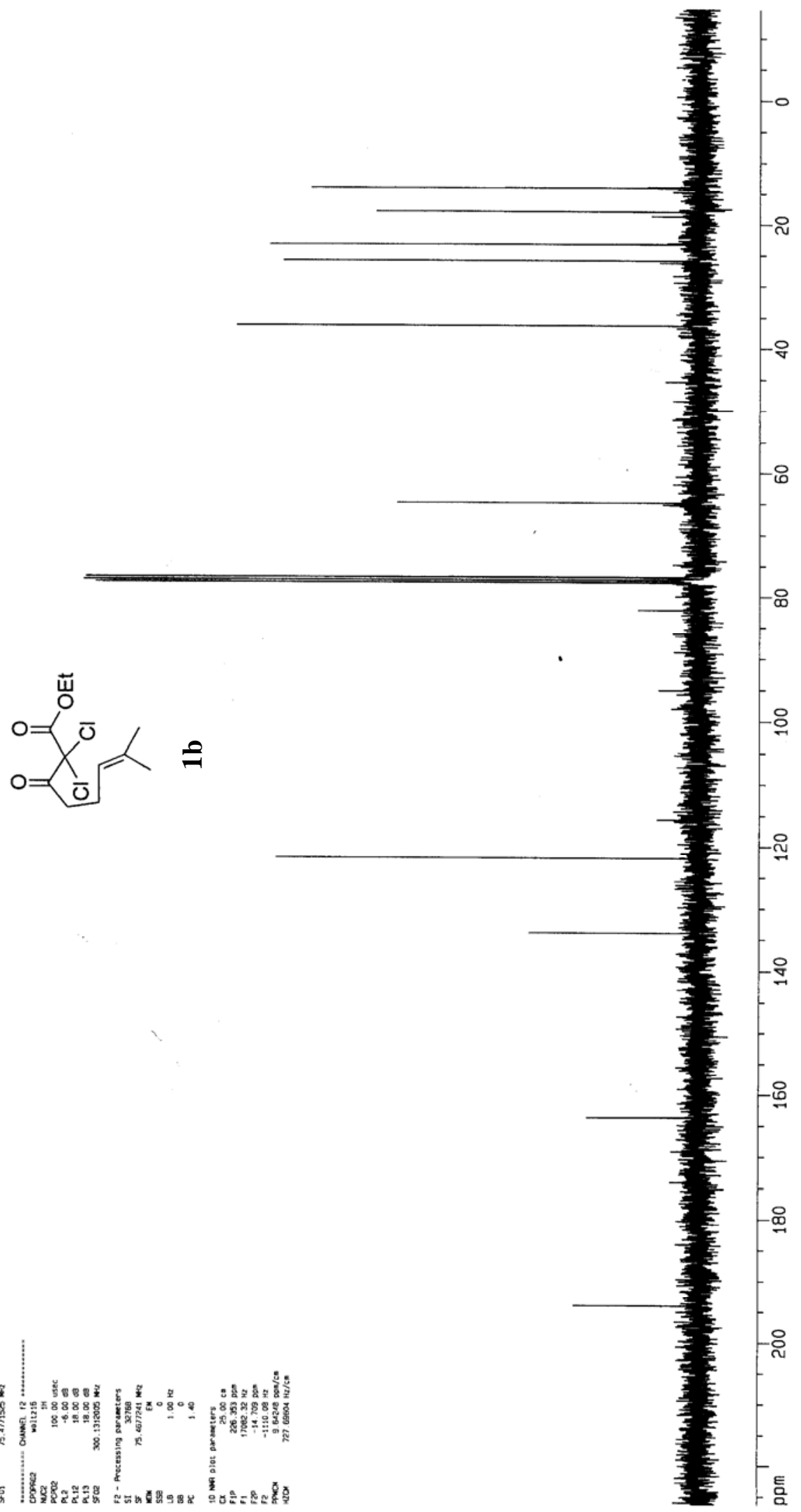

LE6 I I -

$\varepsilon 己 0^{\circ} \nabla \varepsilon$

เ $9 L^{\circ} \varepsilon 9$ -

I $\angle 0^{\circ} \circ 6 \mathrm{~L}$

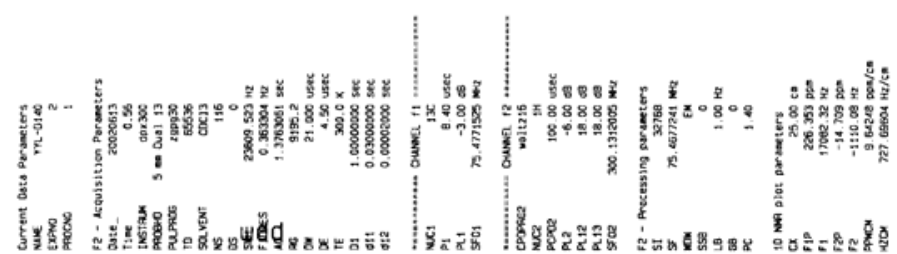




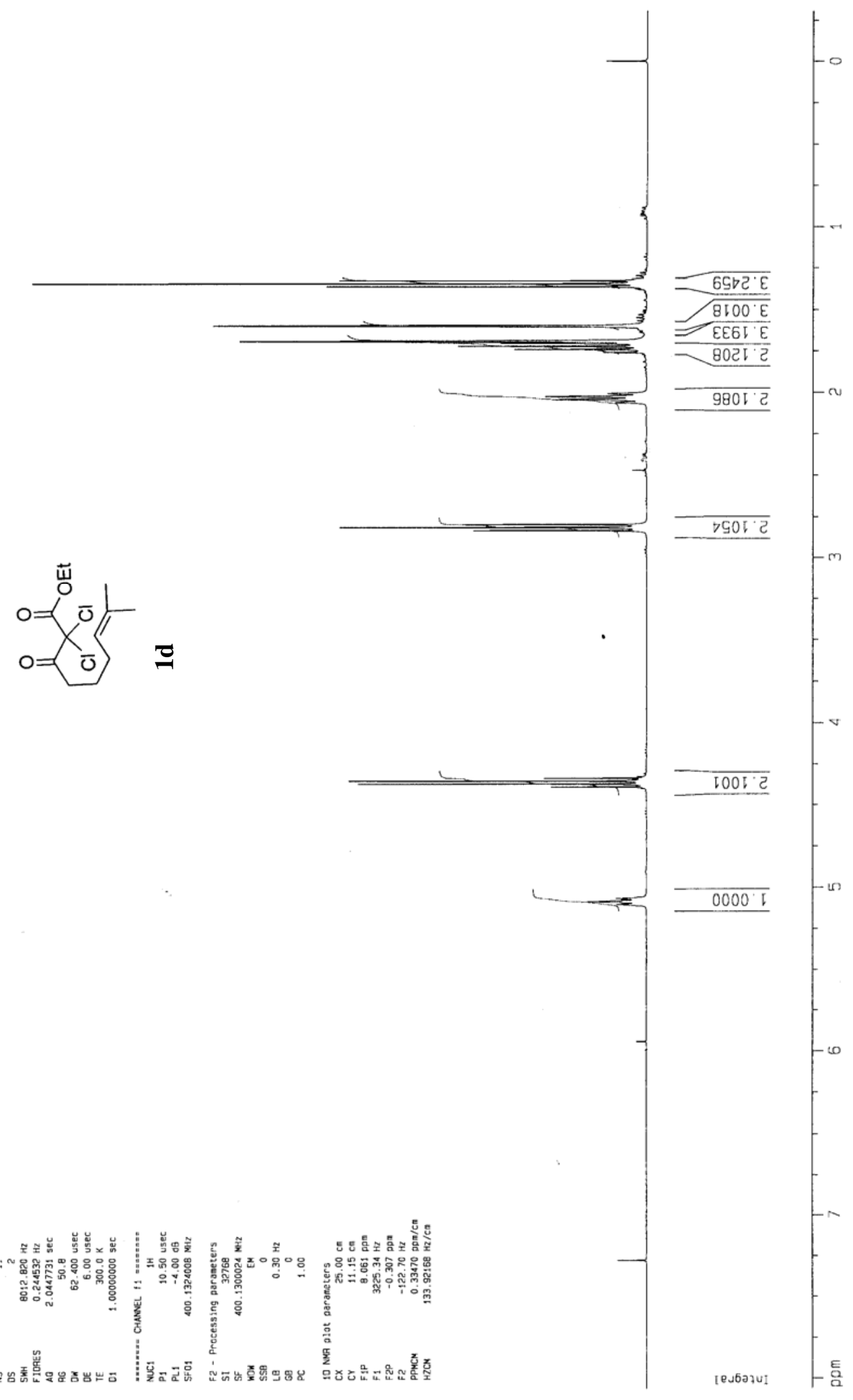



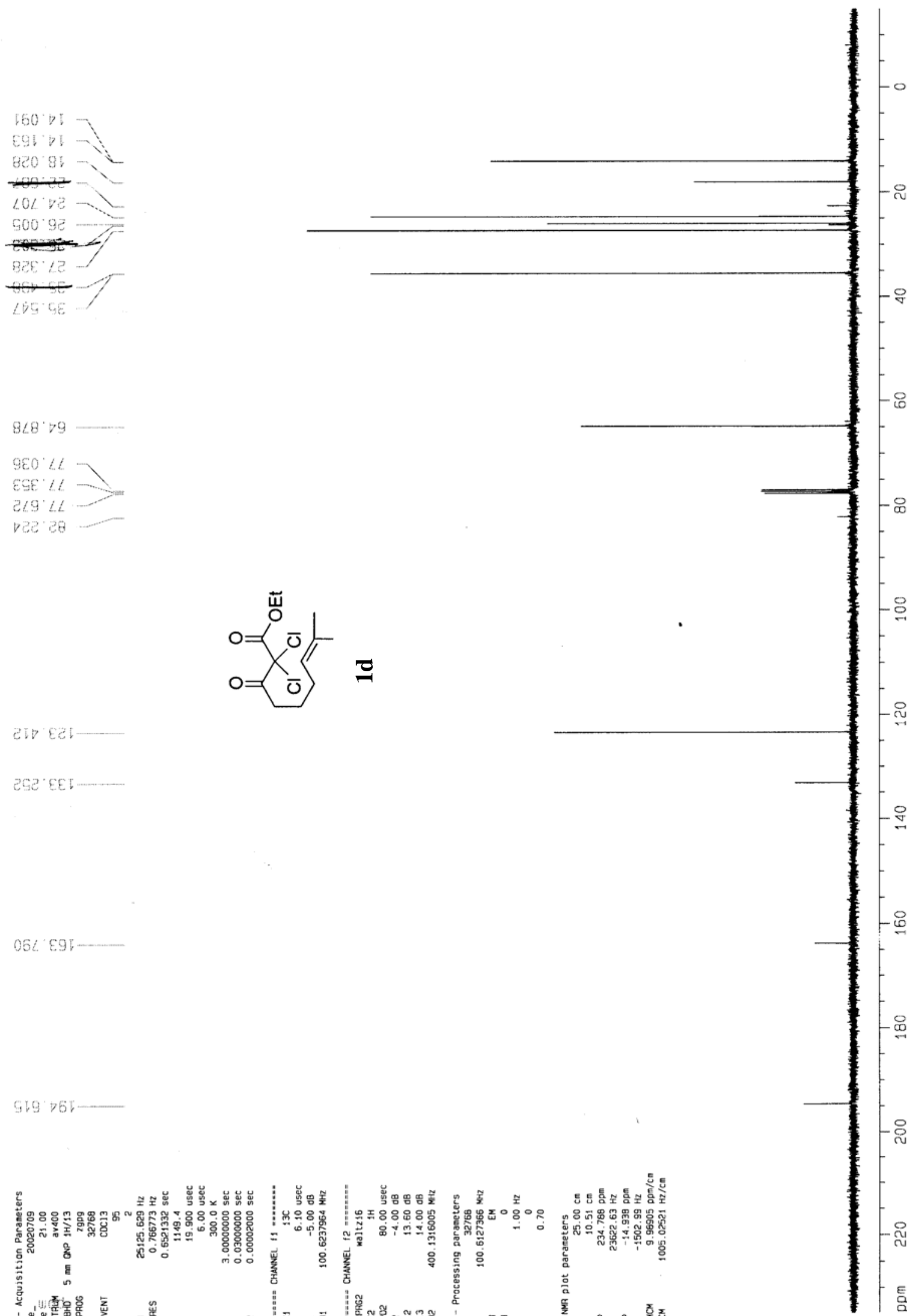

$8 \angle 8^{\circ} 79$

$9 E 0^{\circ} \angle L$

egE $/ t$

2/9'L

$\checkmark 2 e^{\circ} 20$

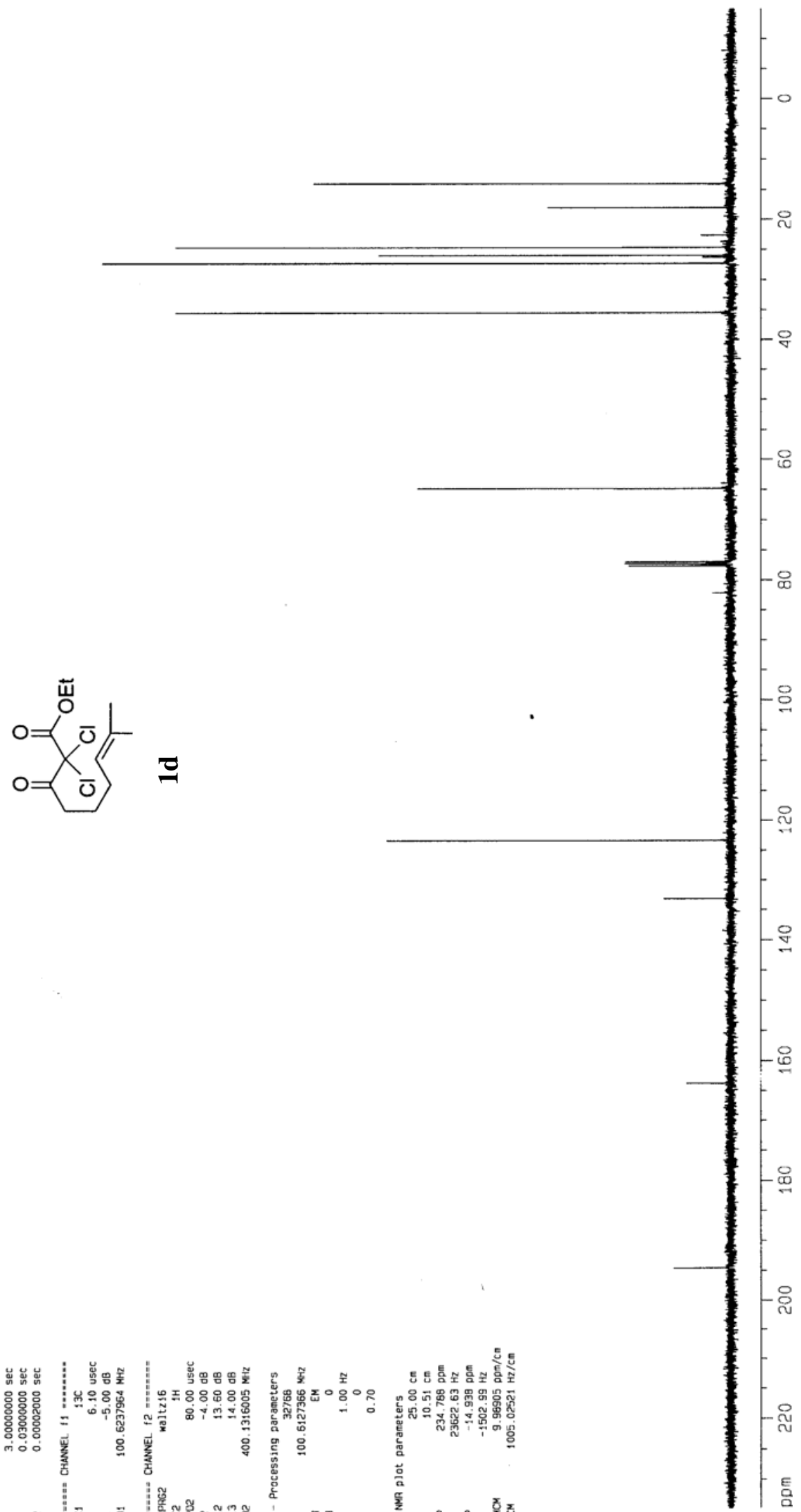

ZTV Ed -

2Ge EEL ..............

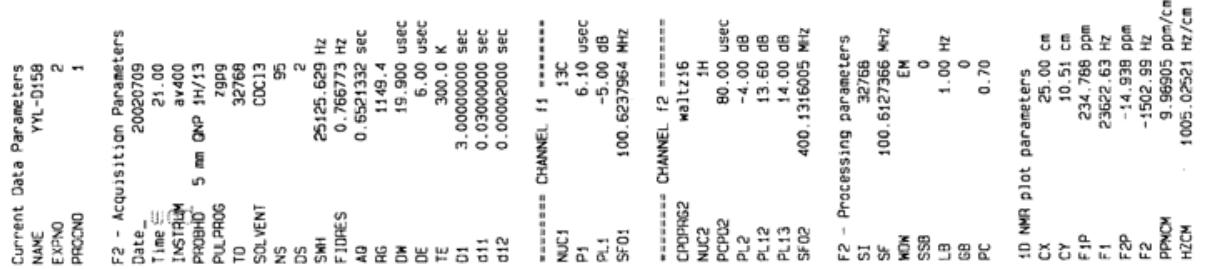




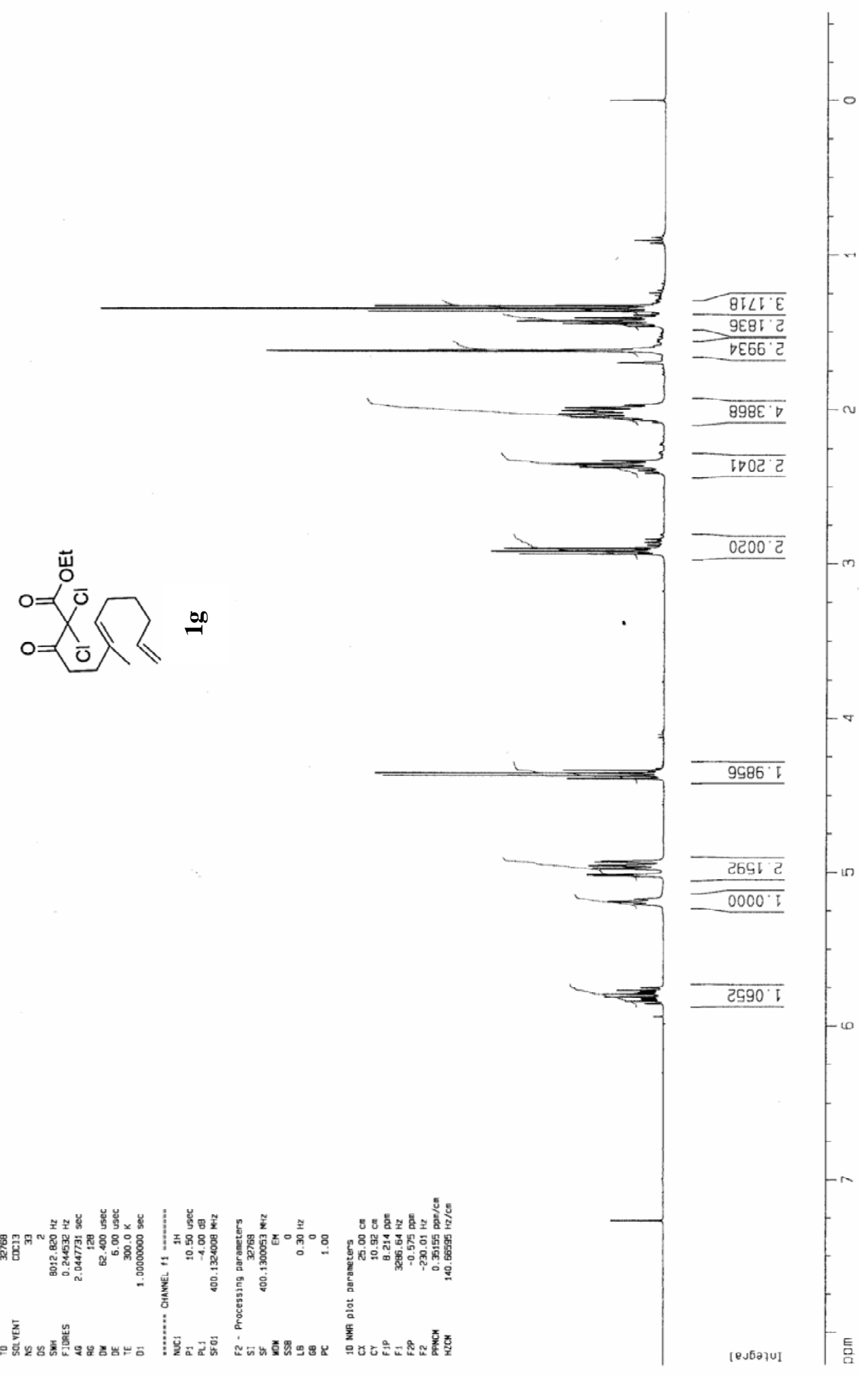




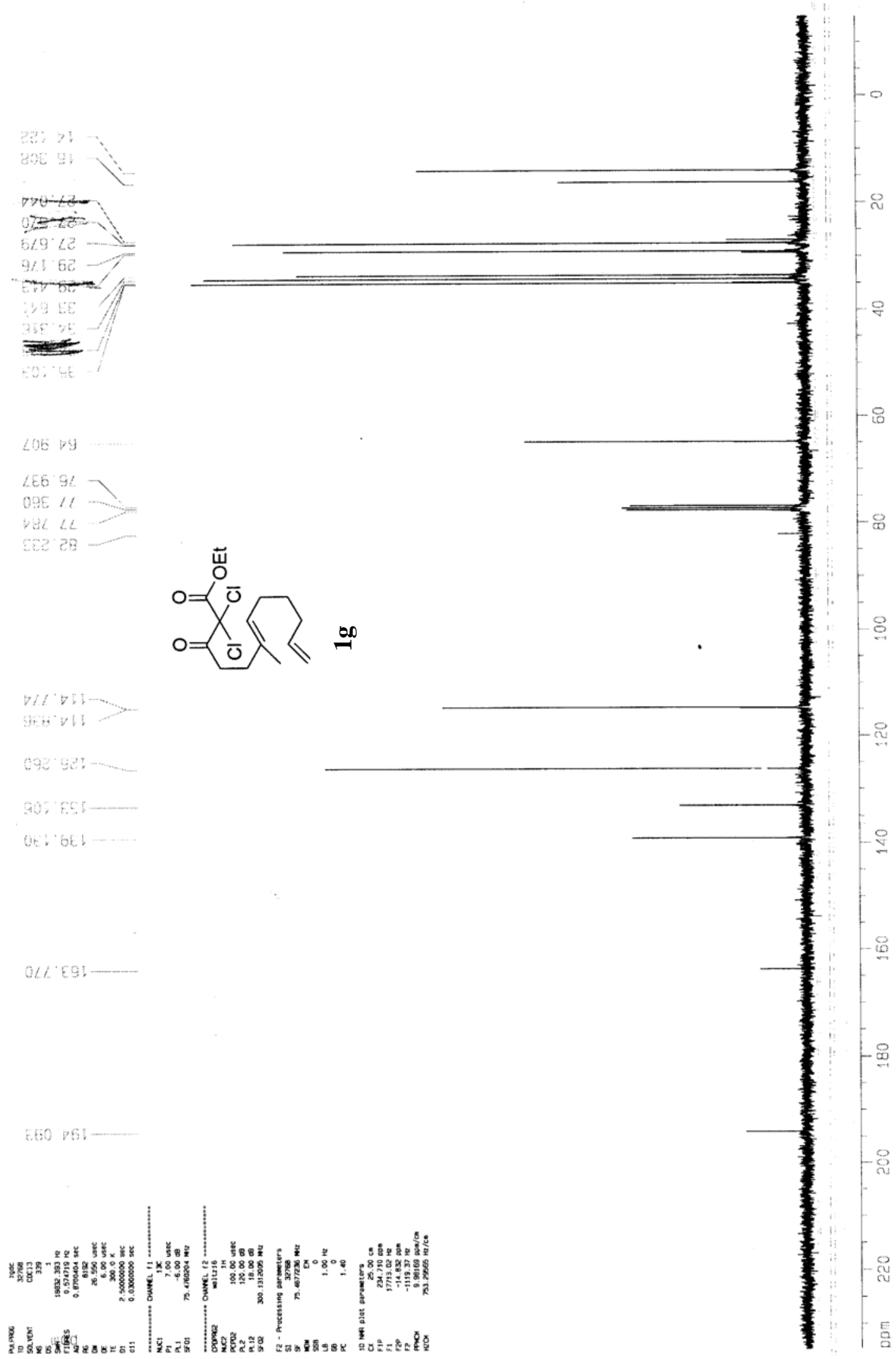




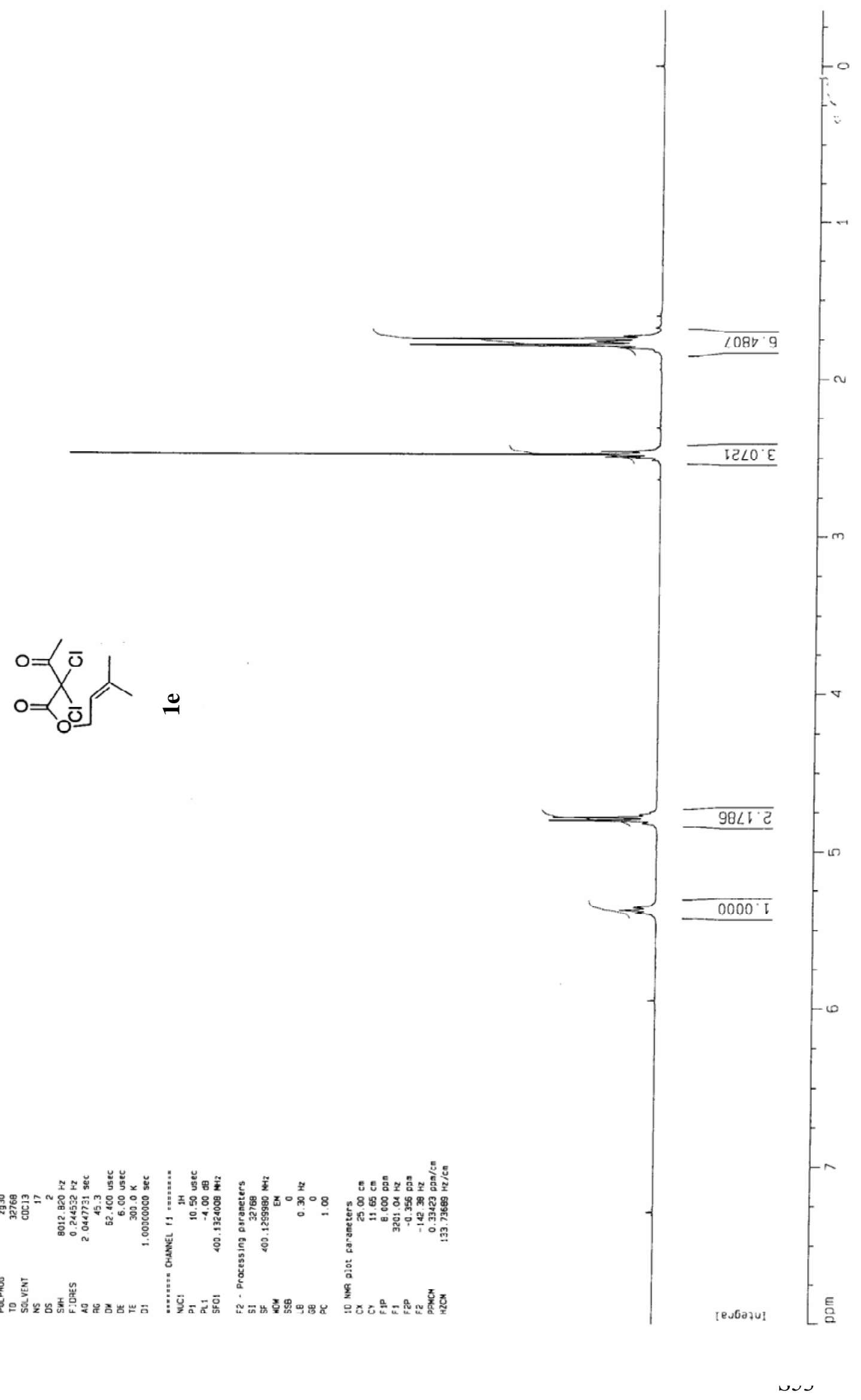



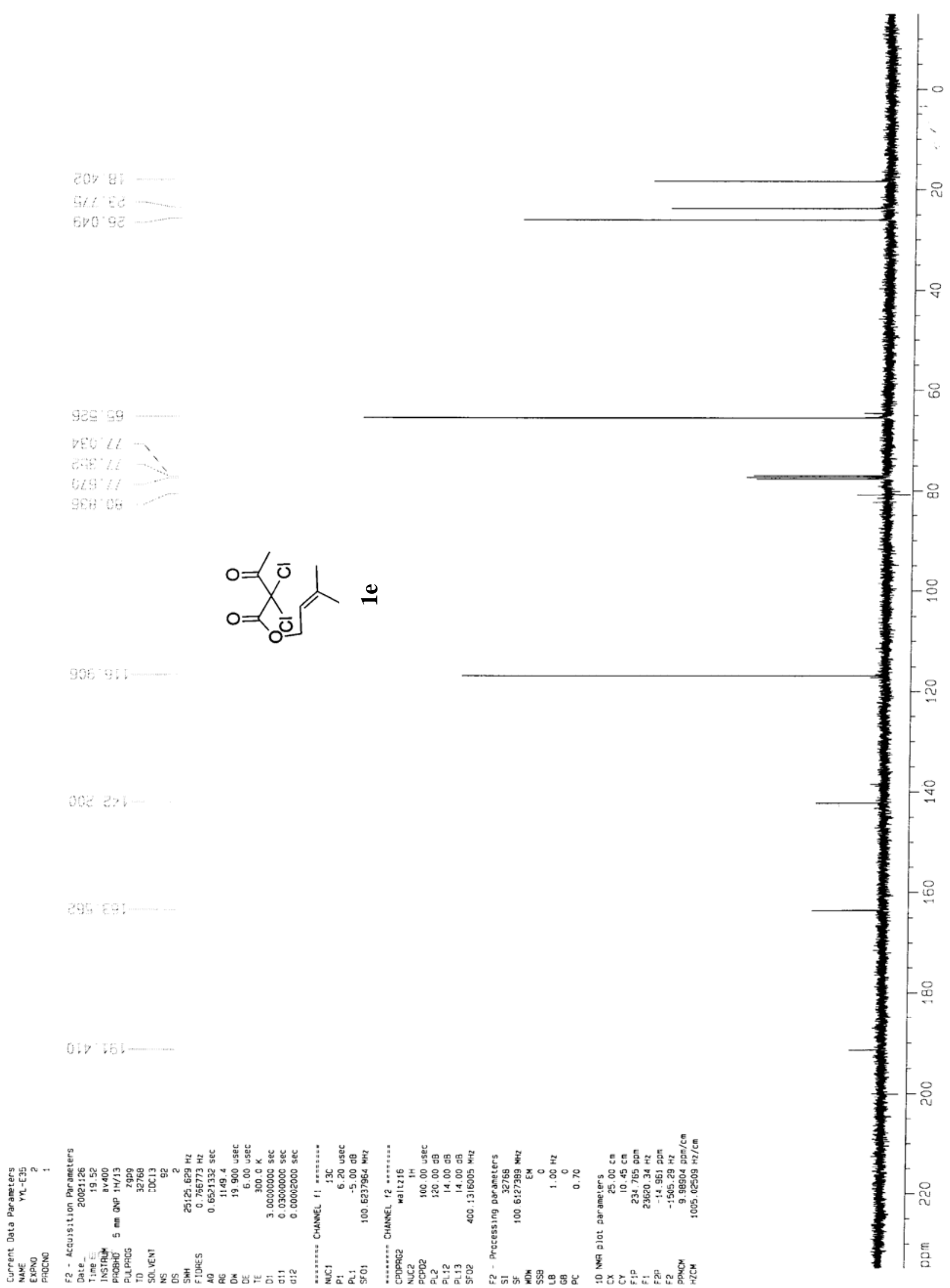


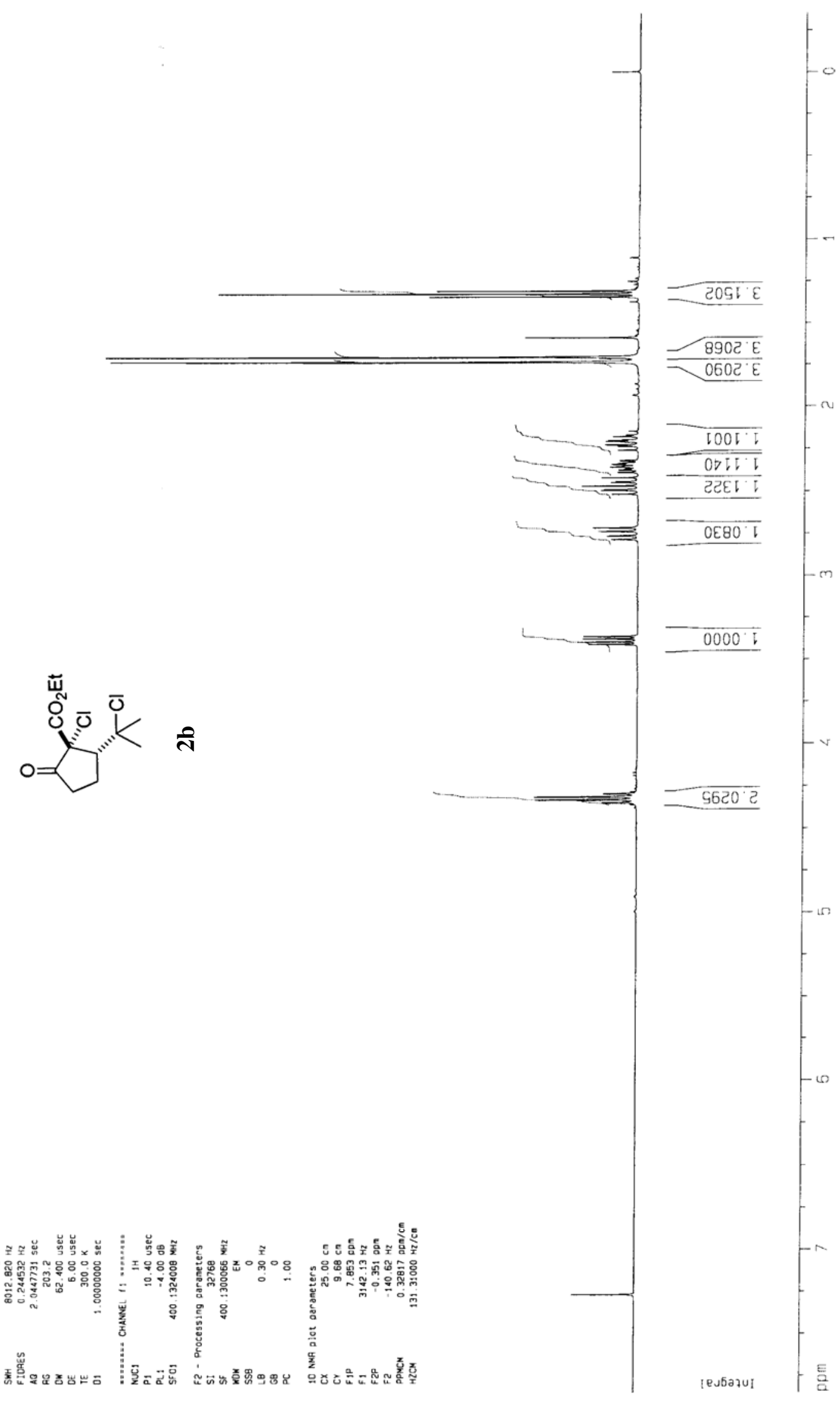




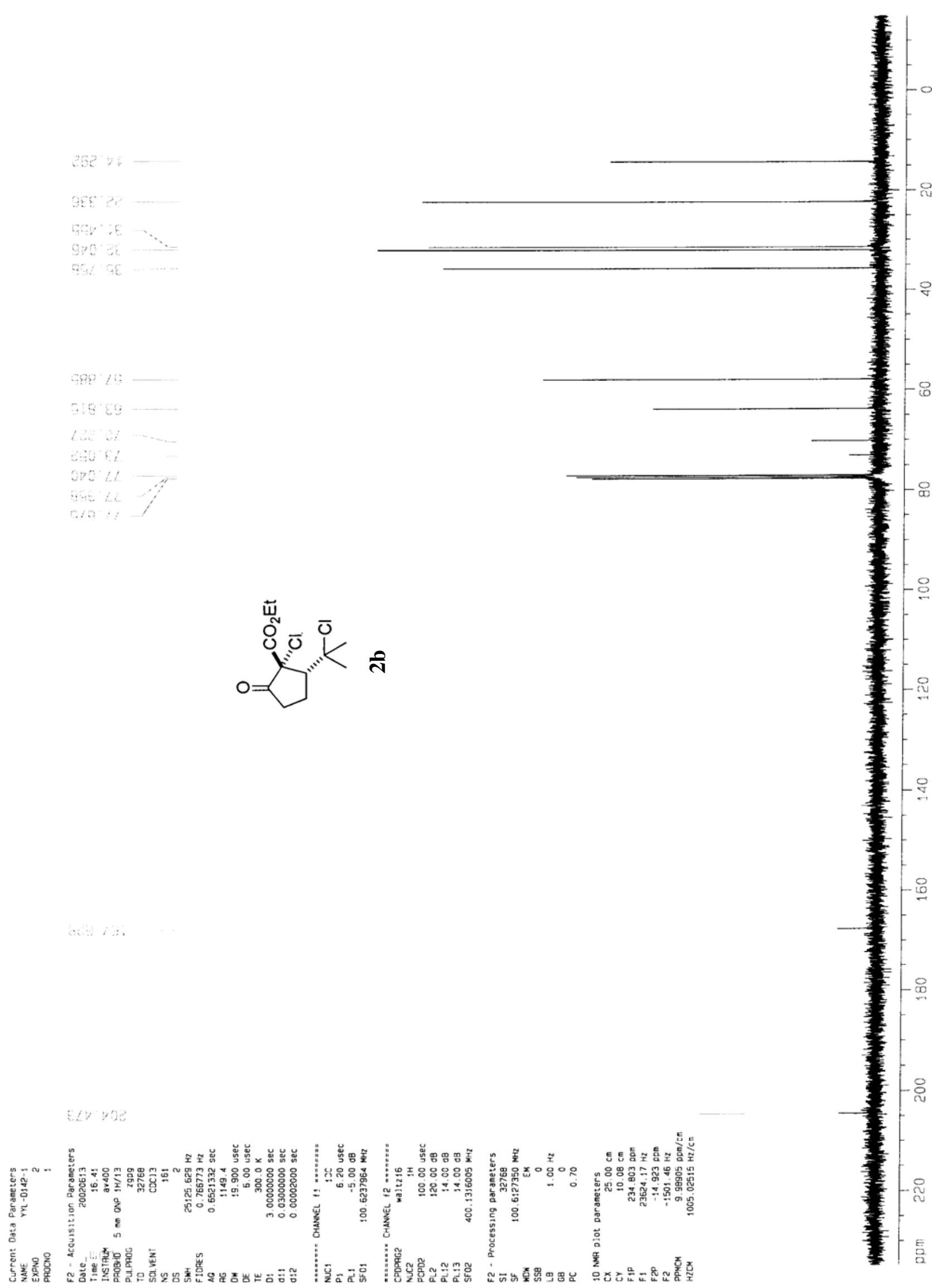




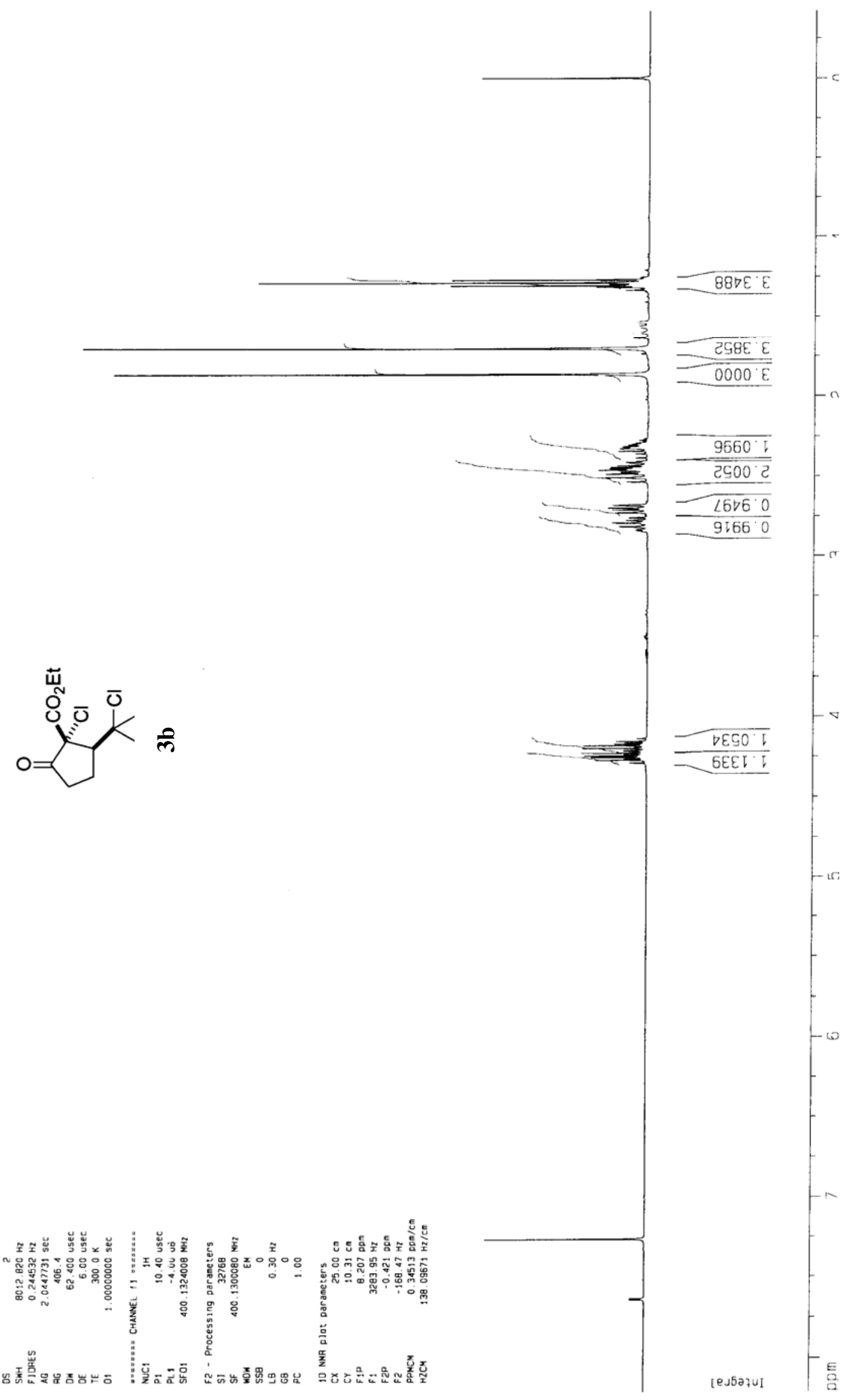




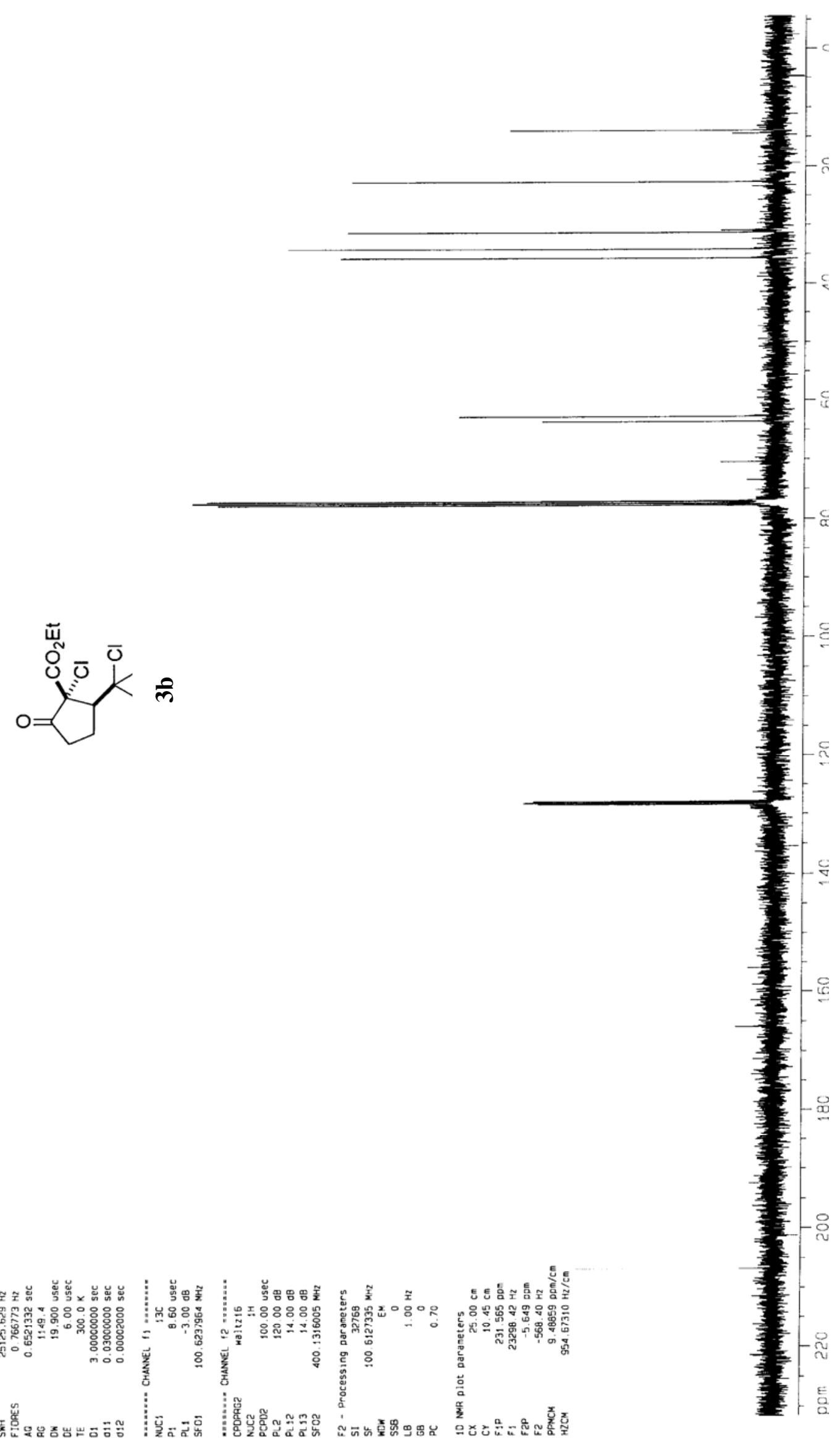




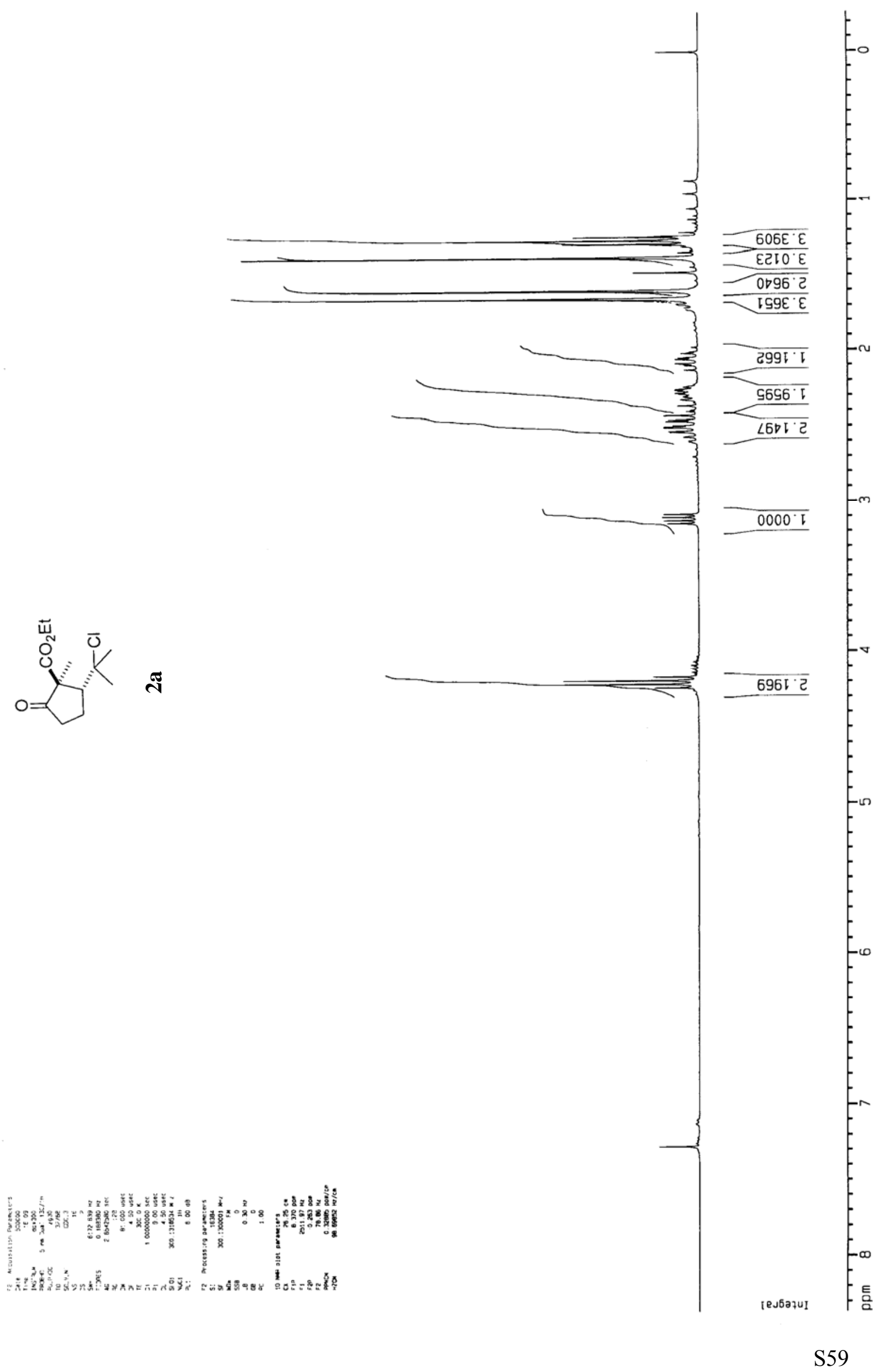



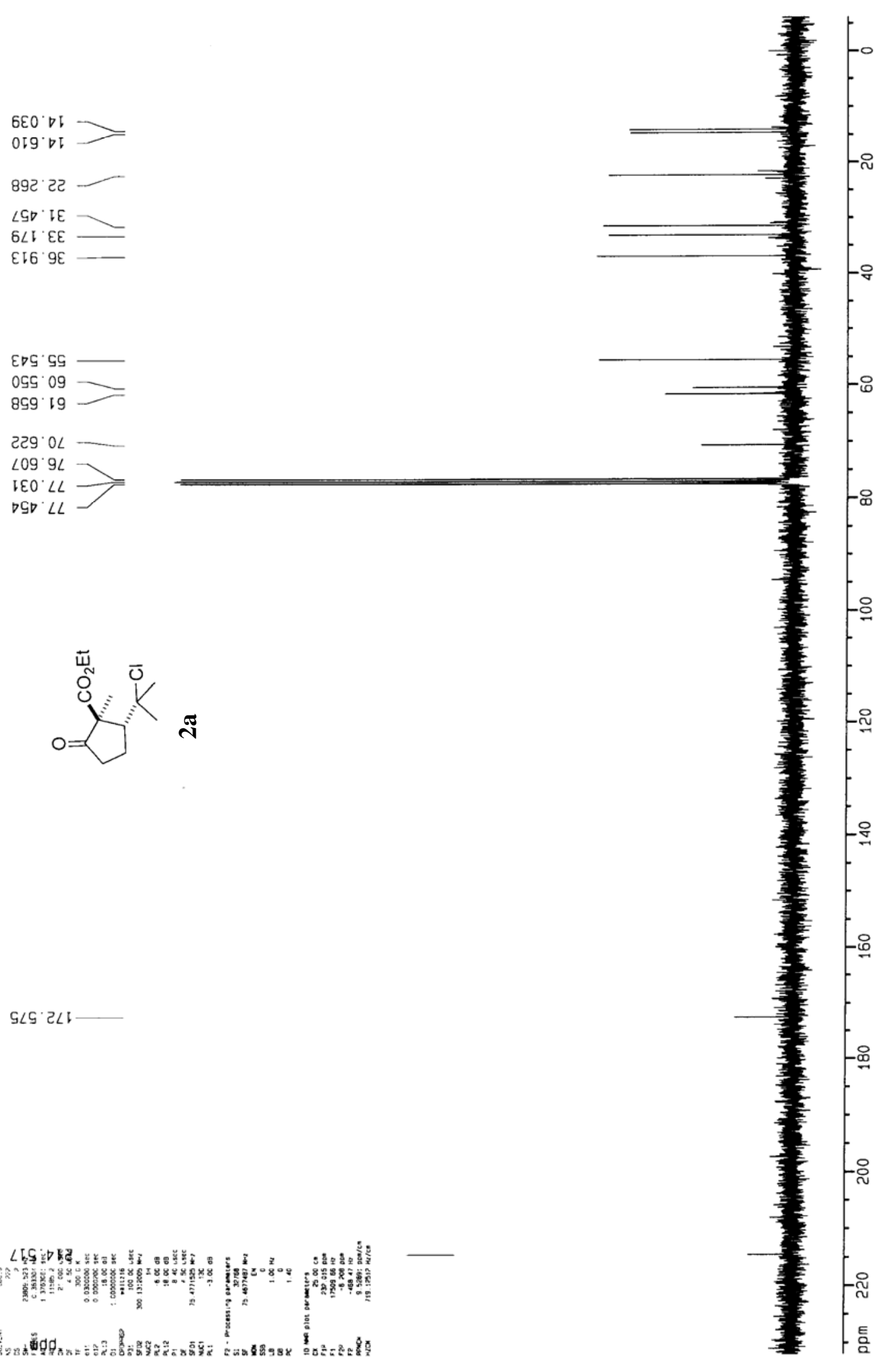

$S \angle S^{\circ} 2 \angle L$

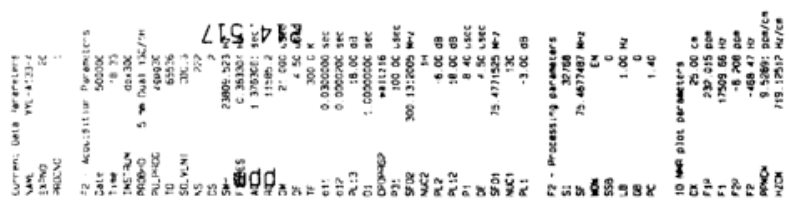




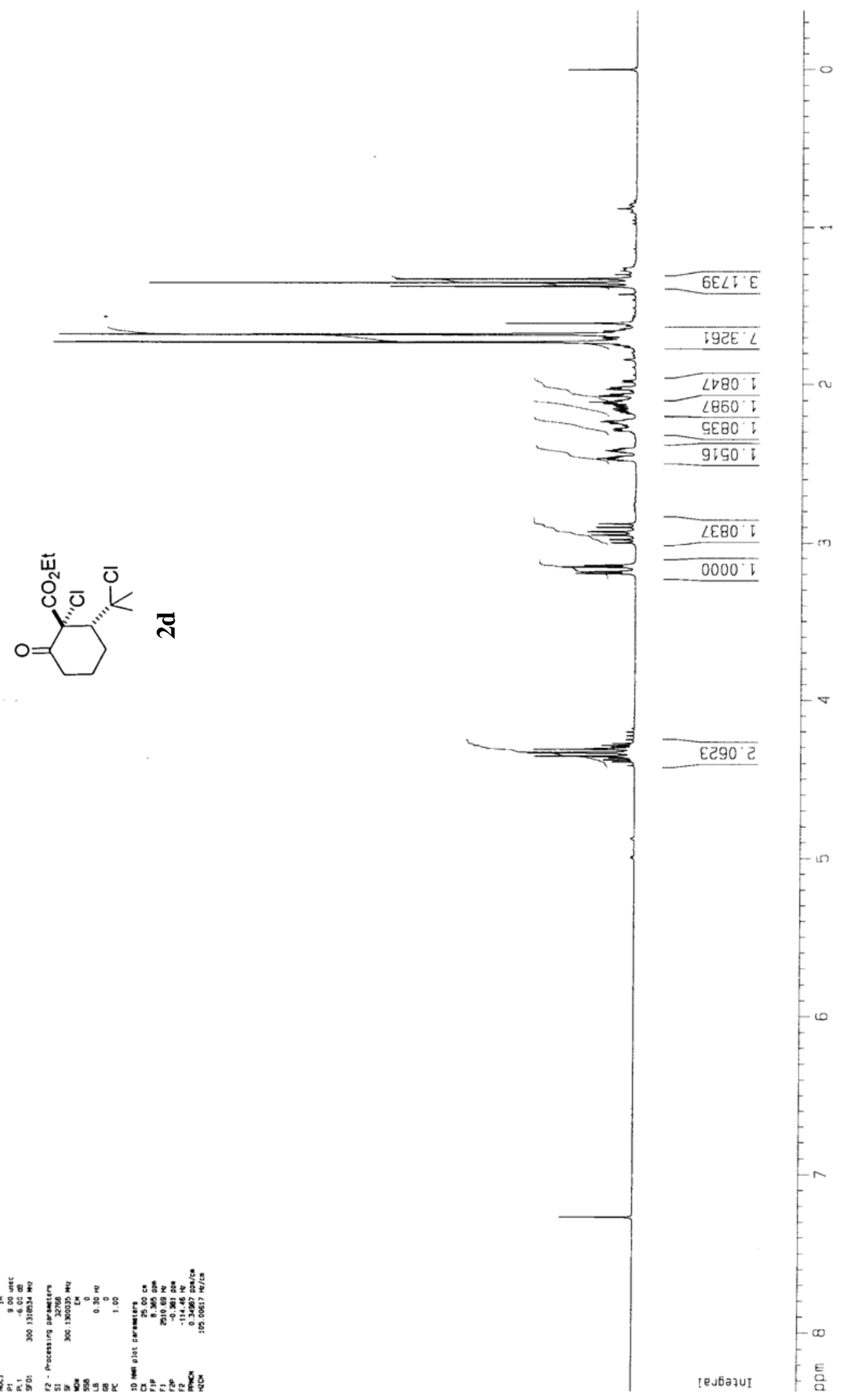




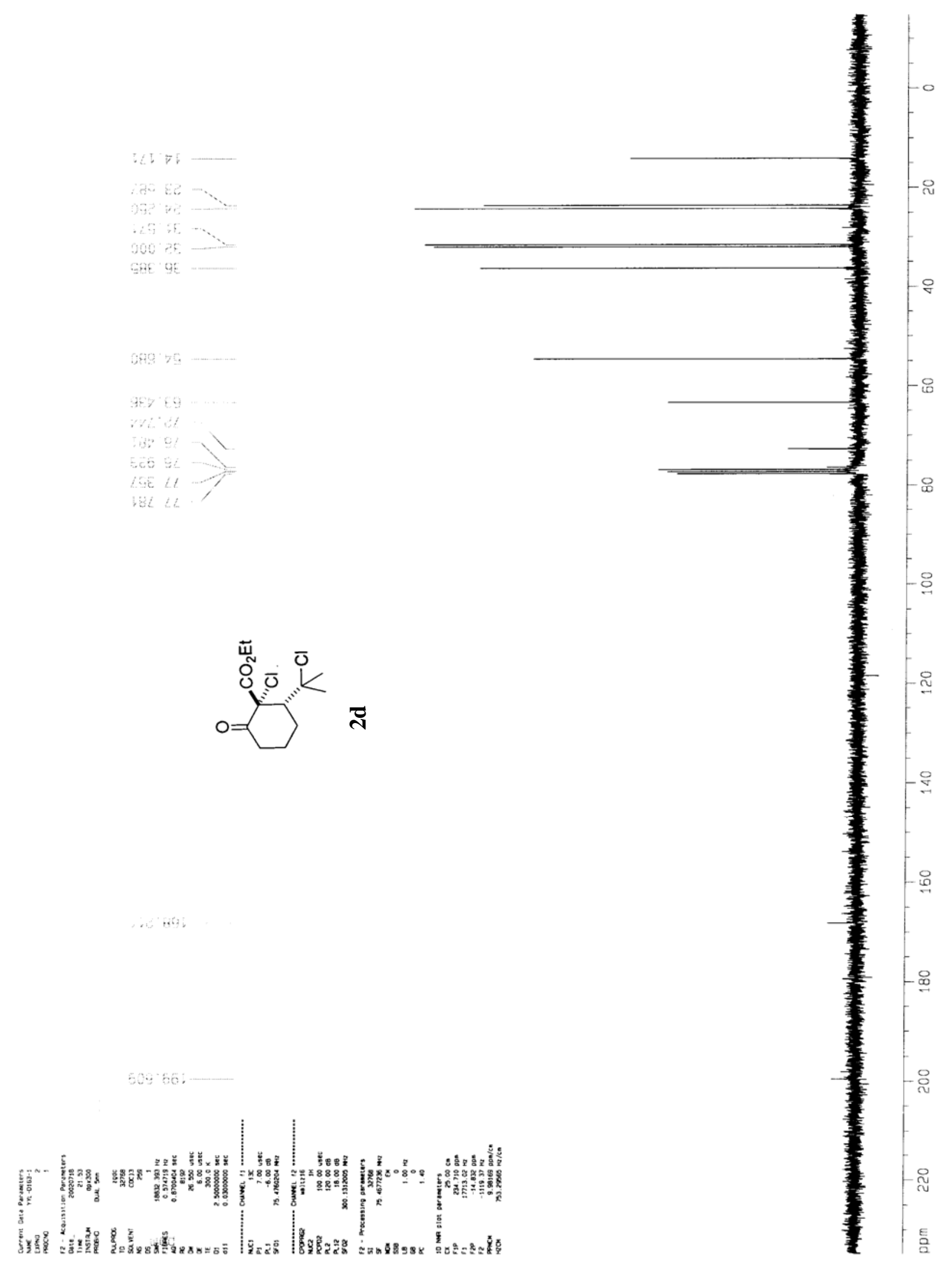




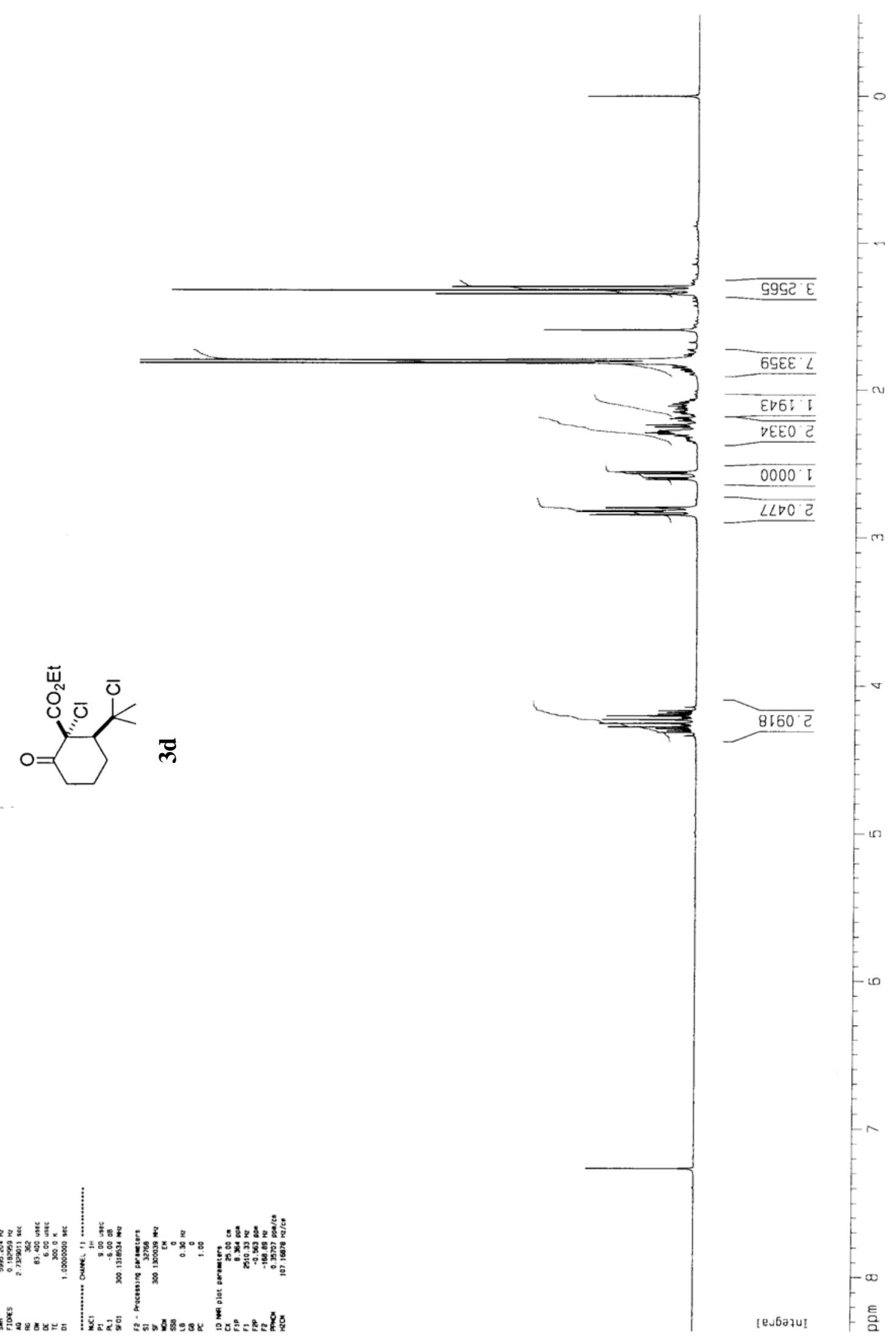




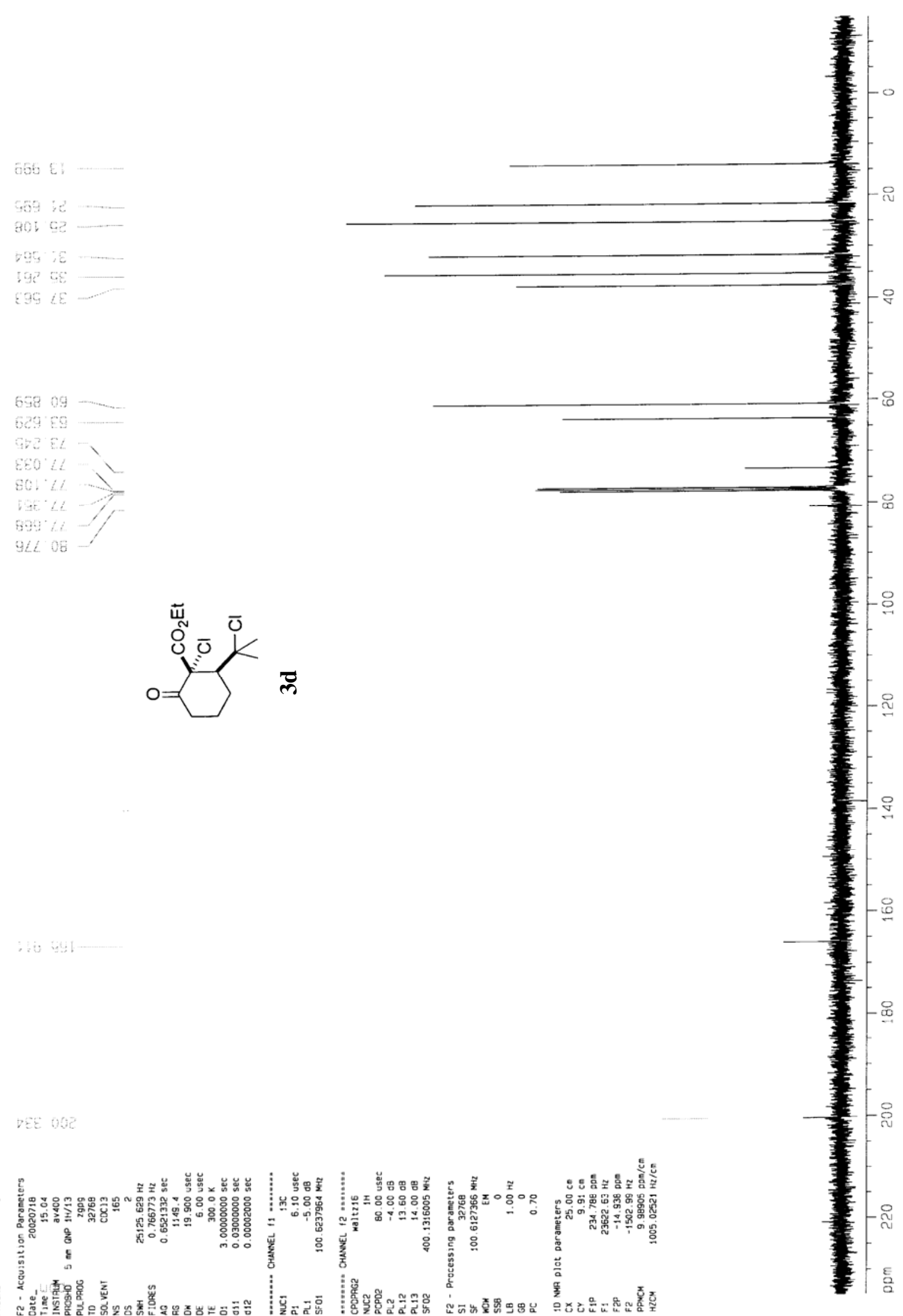




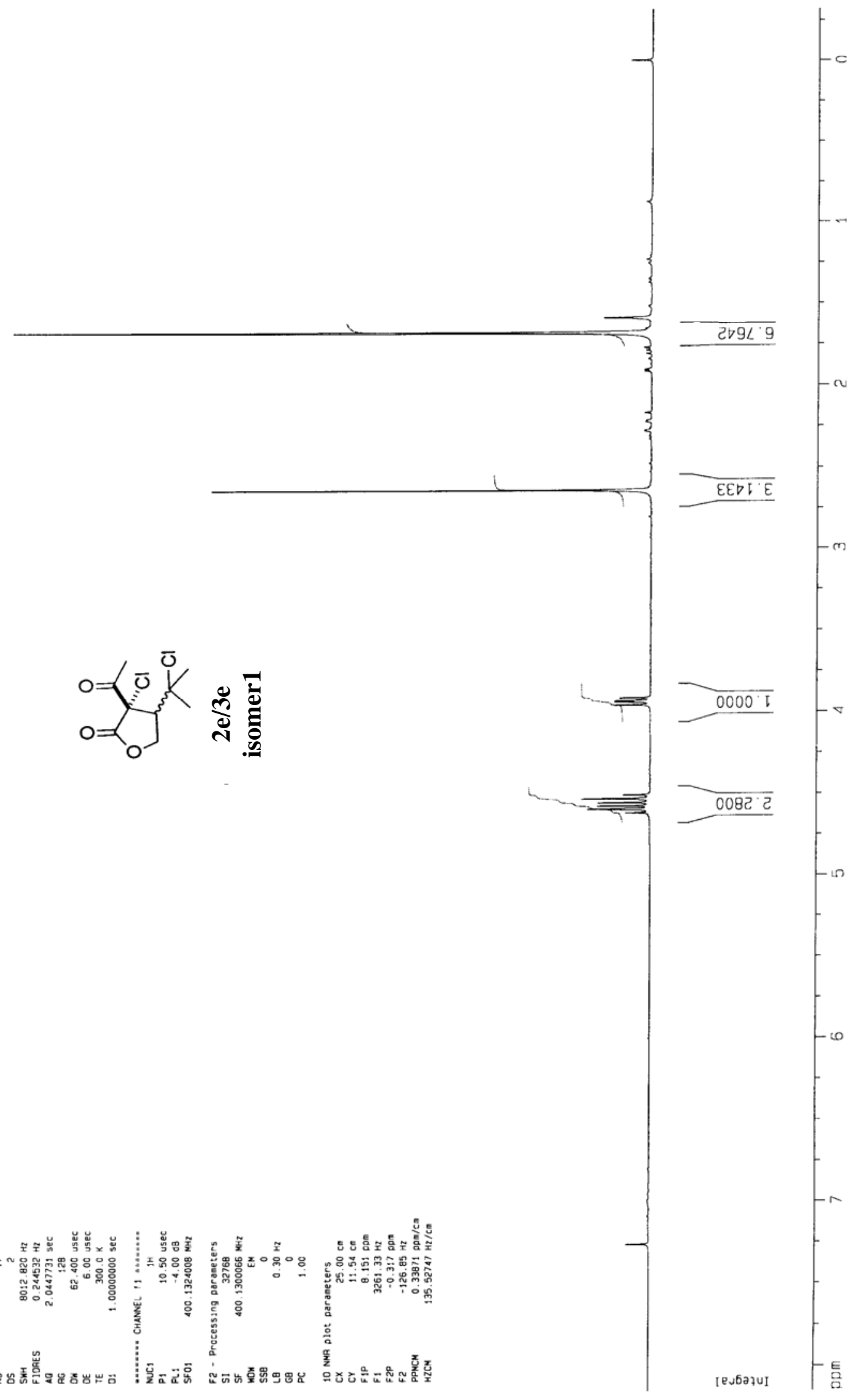




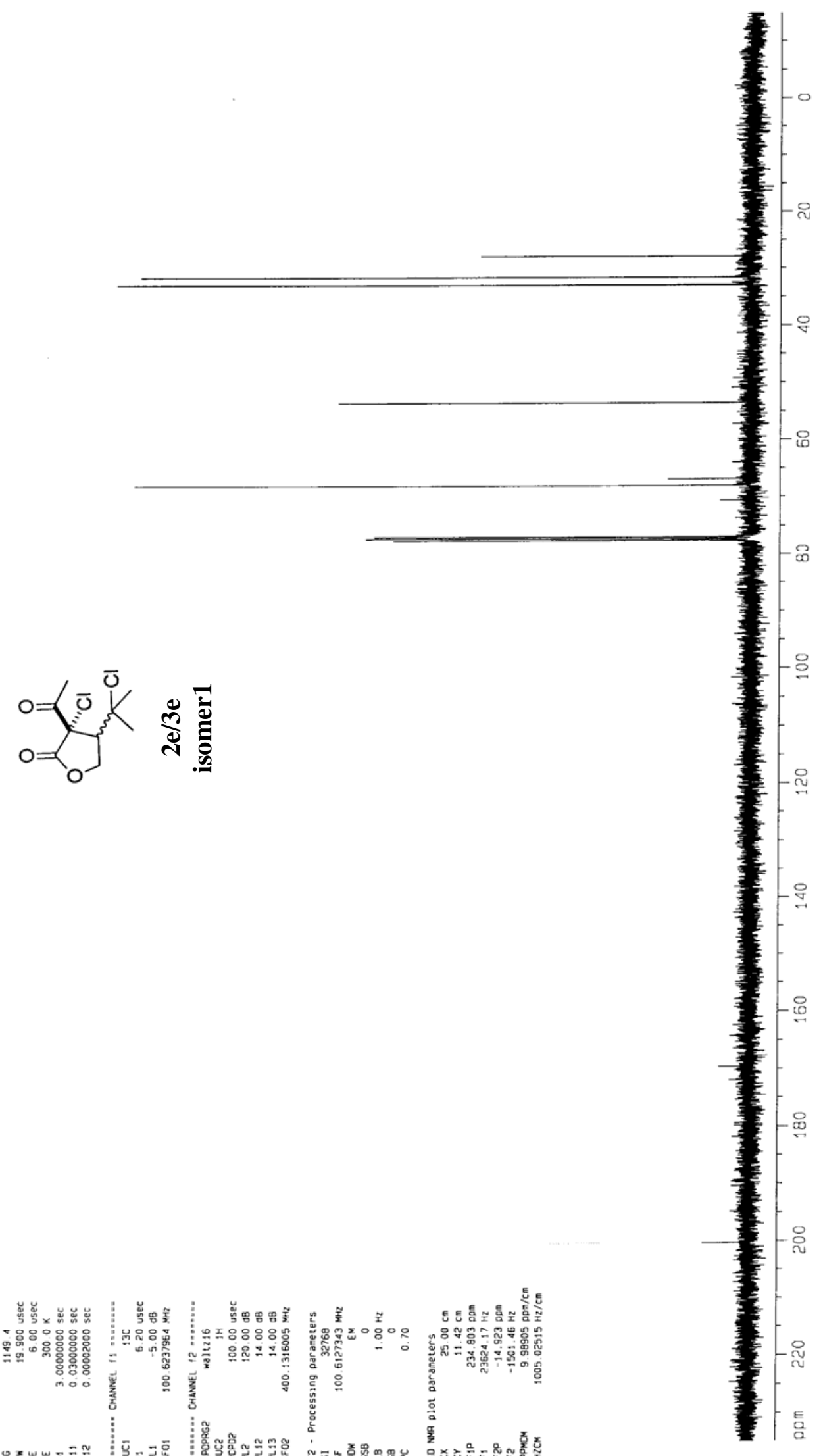

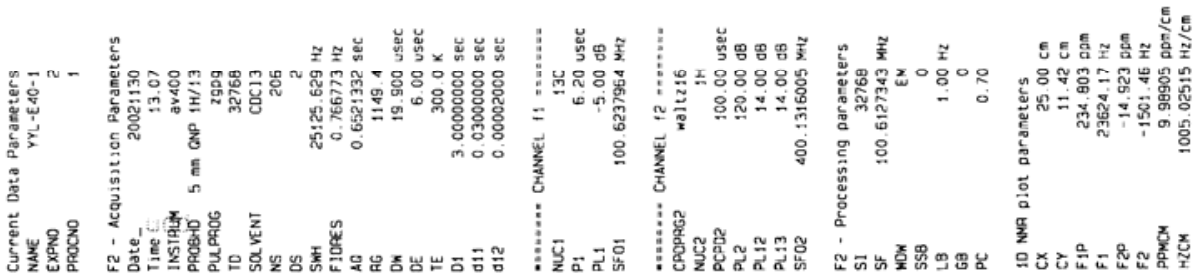




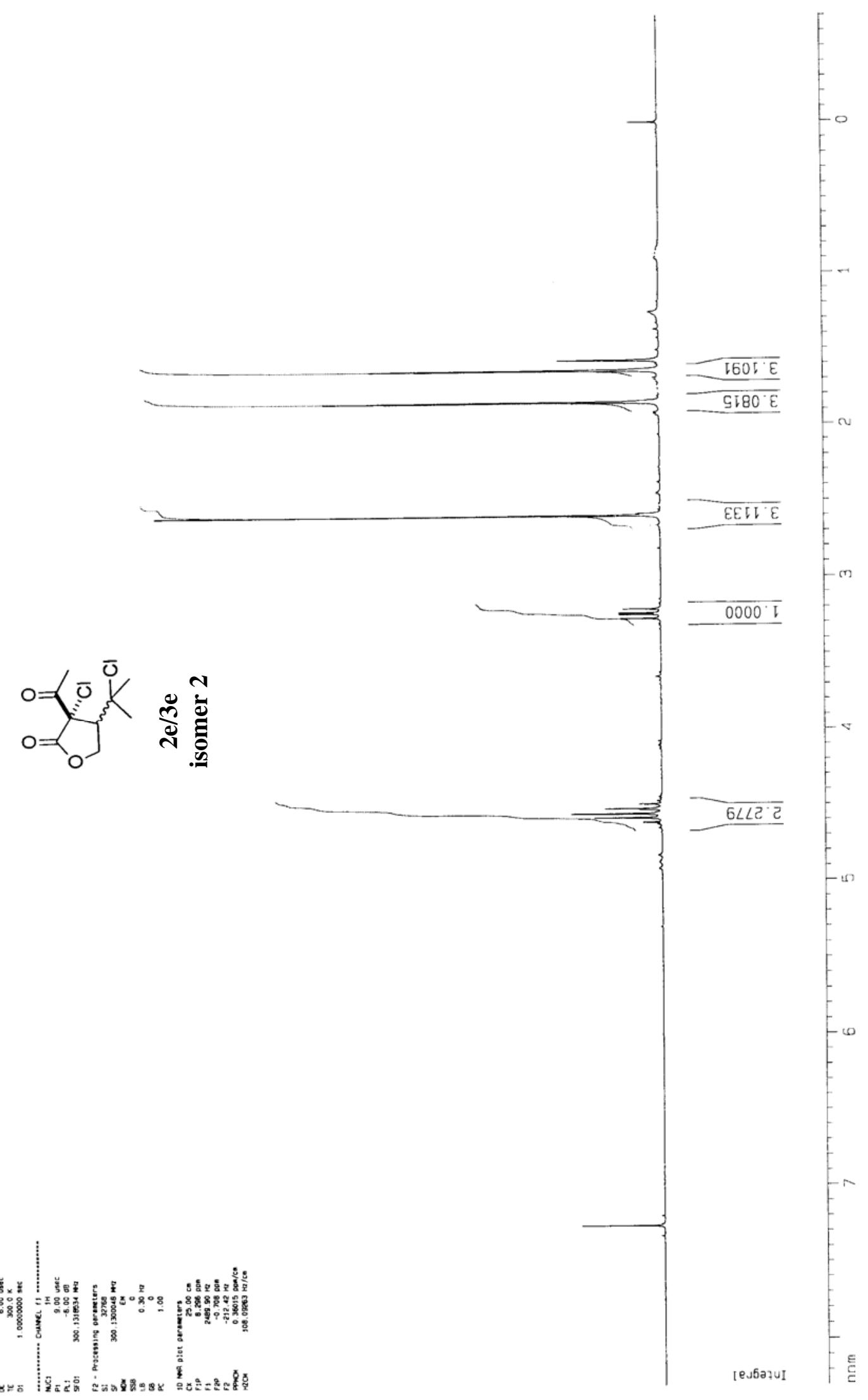




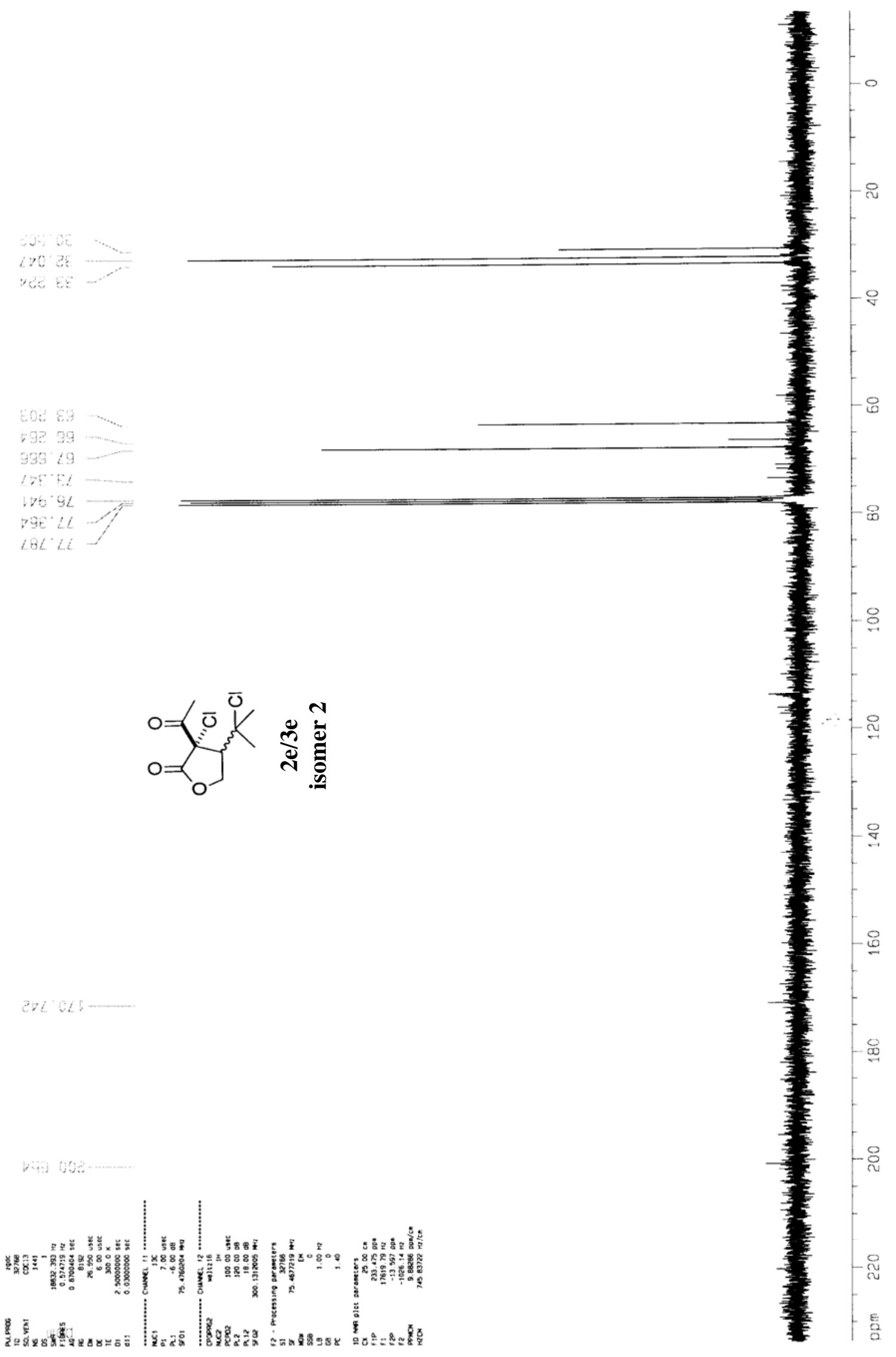




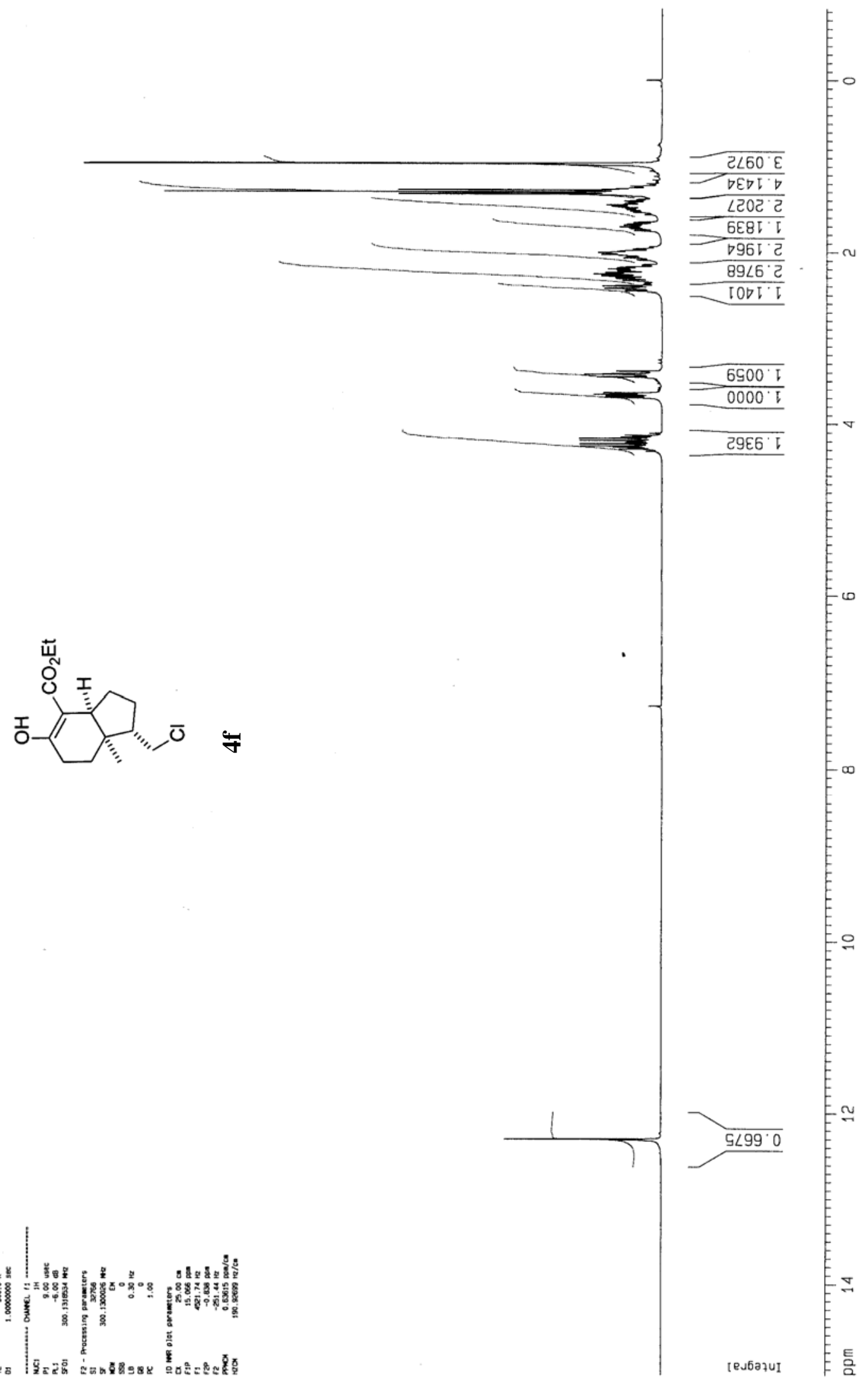




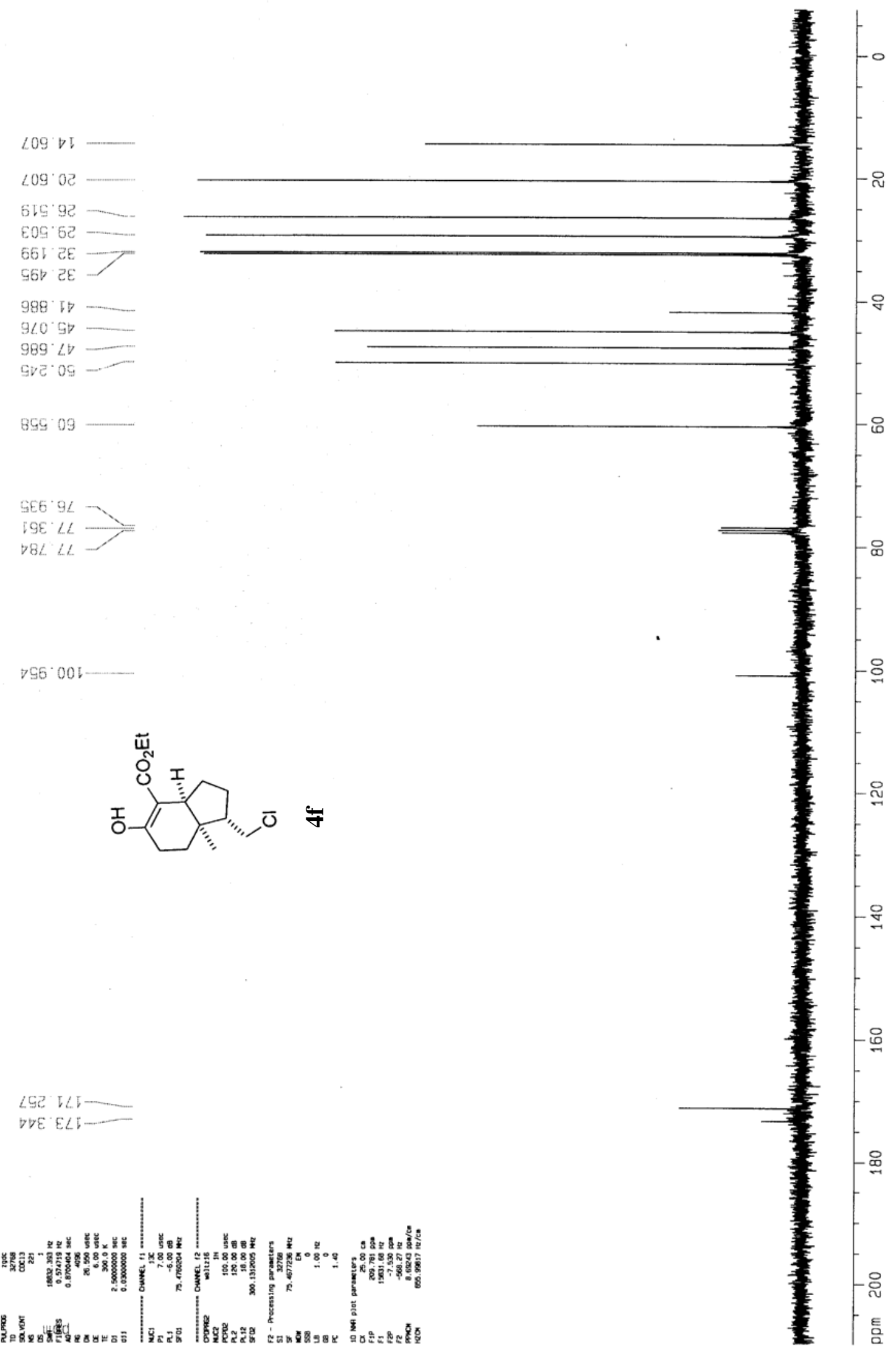




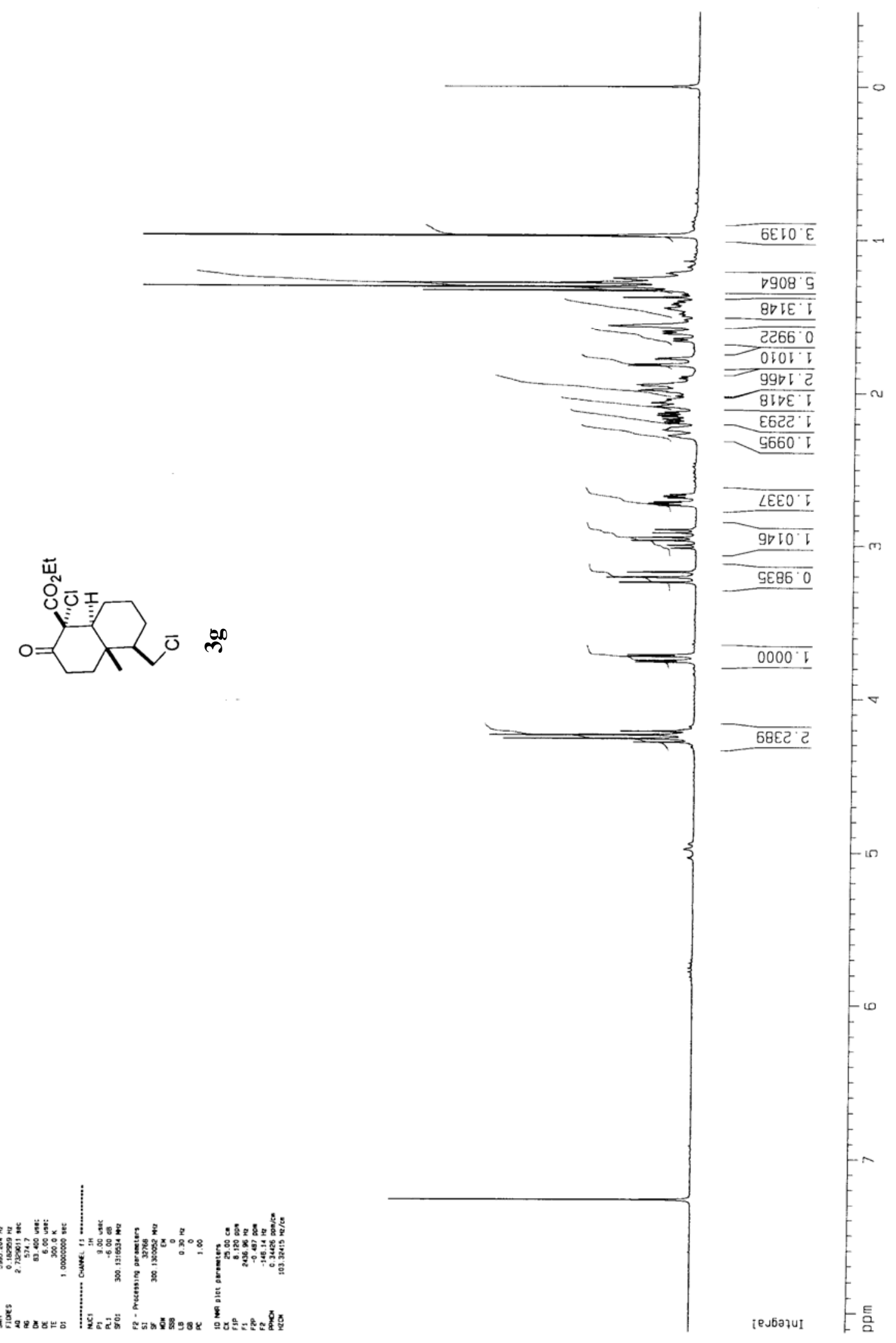



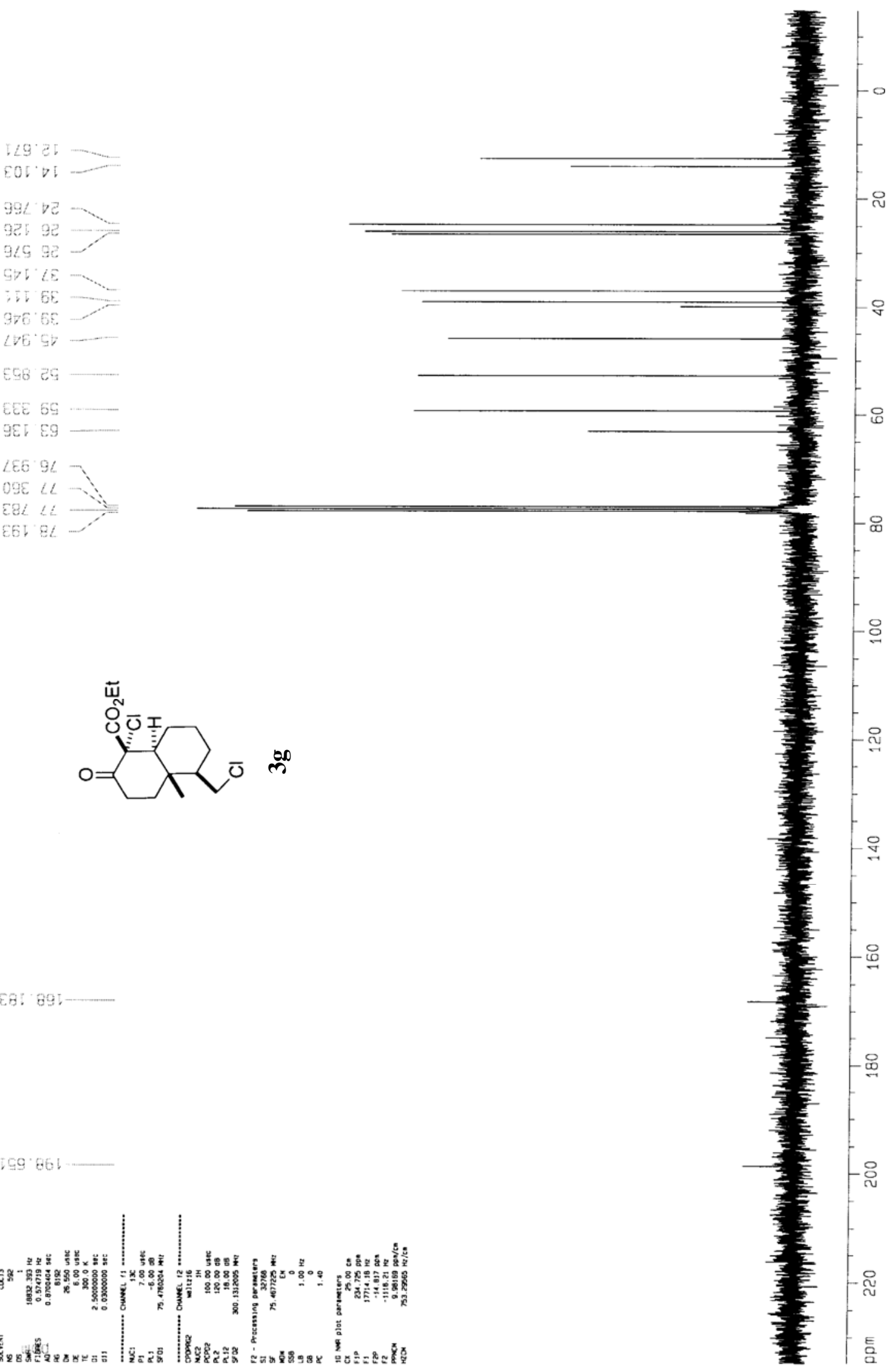

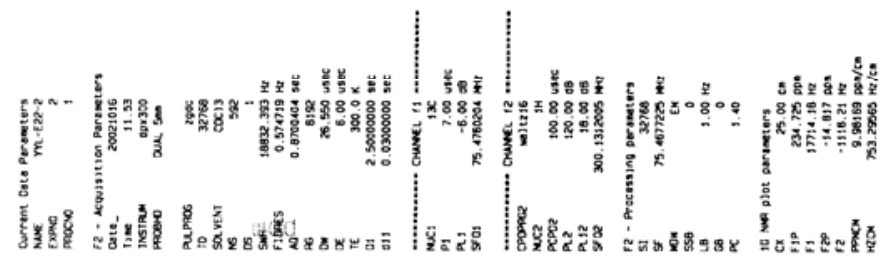




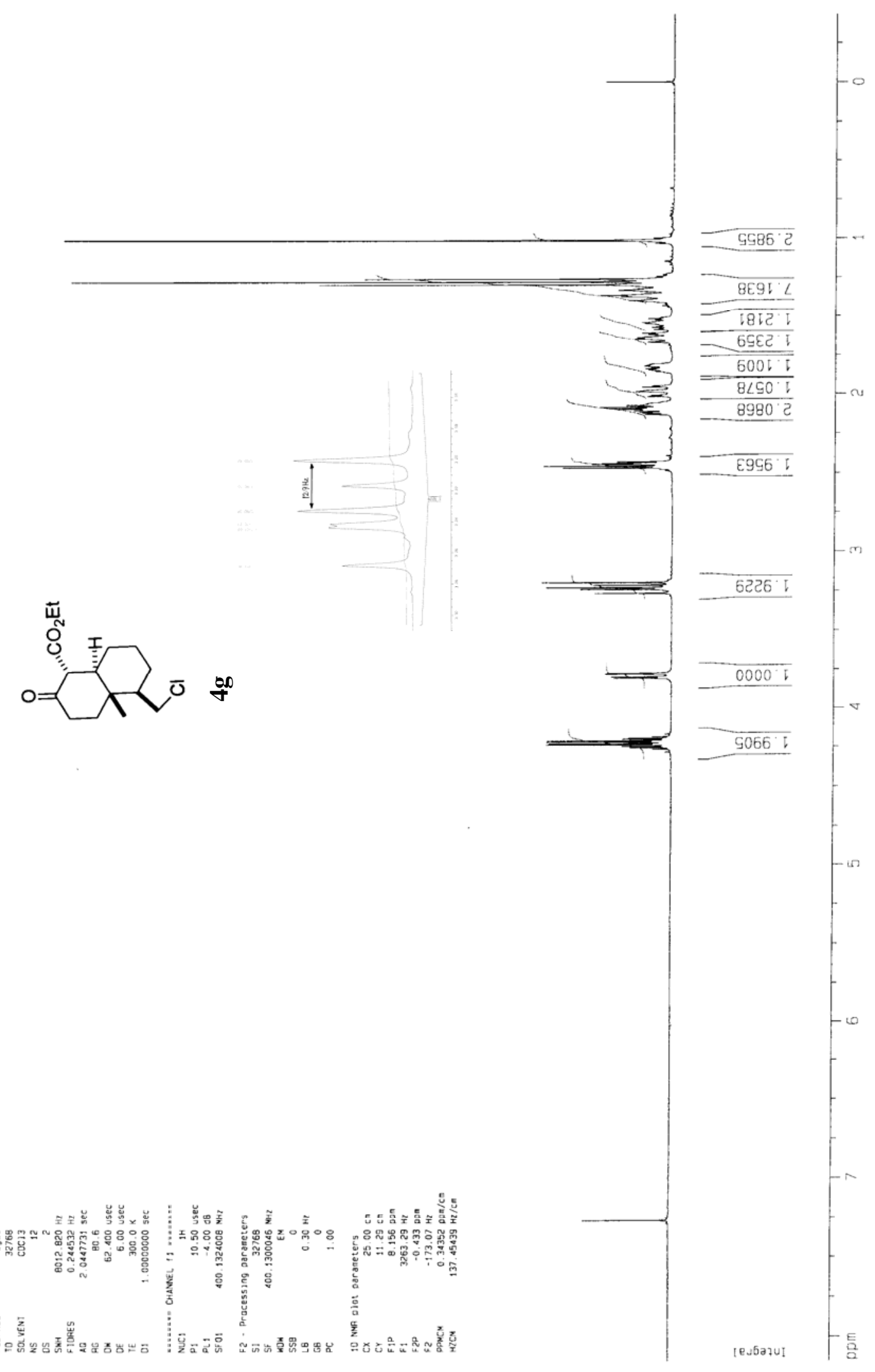




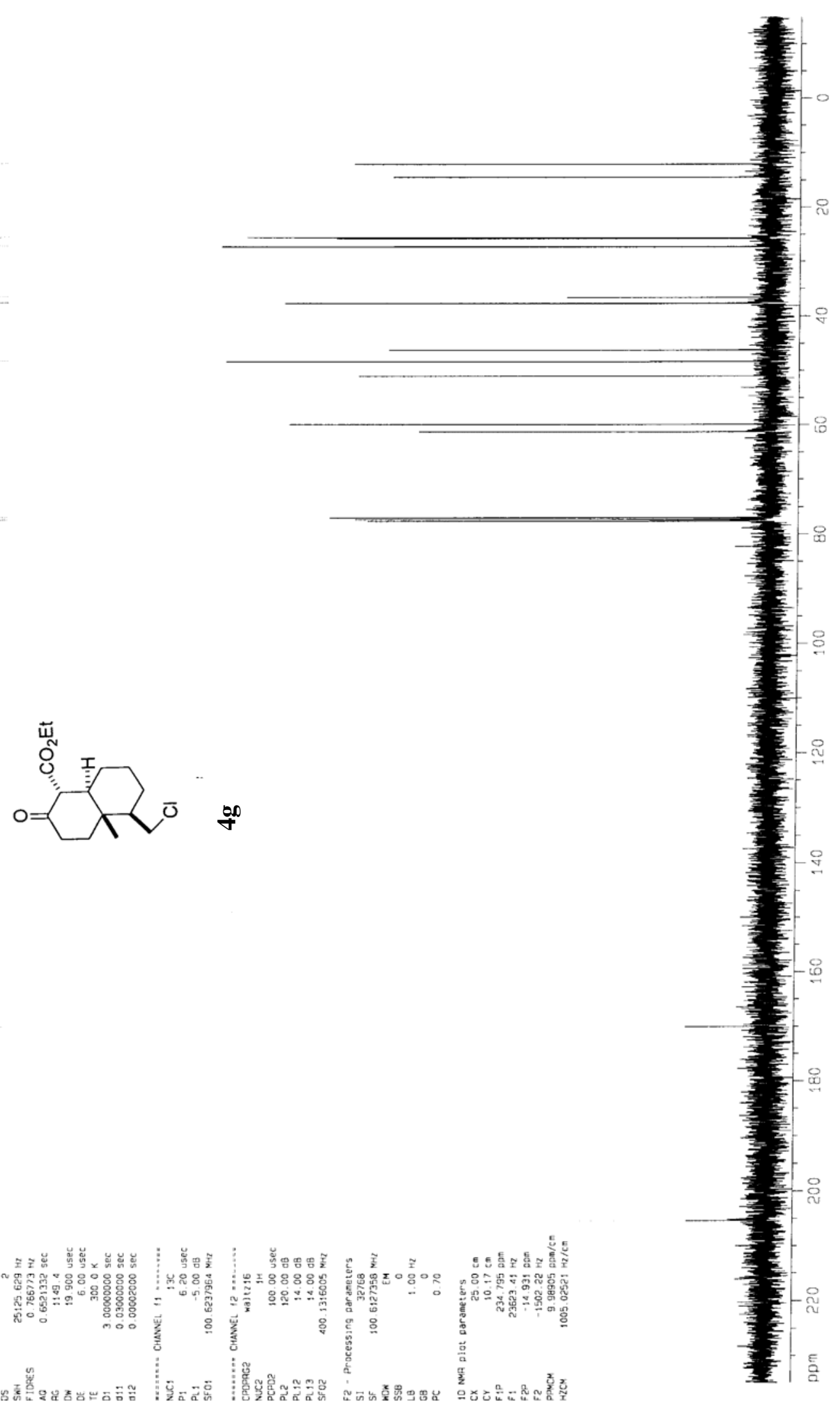

